\title{
An Investigation into the Dynamic Response of Vocal Folds
}

\author{
by
}

Hai Lan

A Thesis Submitted in Partial Fulfilment of the Degree of Master of Engineering

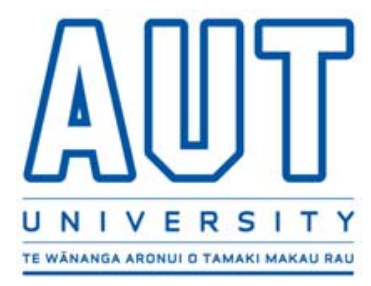

Auckland University of Technology

Auckland, New Zealand

May 2006 


\section{ACKNOWLEDGEMENT}

There are many people I wish to formally thank for their contribution to this thesis. First of all, I would like to express my appreciations and gratitude to my supervisor, Professor Ahmed Al-Jumaily. He is such knowledgeable, patient and helpful. In the two years master study, I learned more than I expected from him. Without his invaluable support, consistently encouragement and push, I will not finish the research like this.

I am grateful to thank Dr. Ali Mirnajafi (Research \& Development Edwards Lifescience in USA) for his suggestions during the course of the investigation.

I wish to thank Dr. David Parker and Dr. Robert Paxton for correcting this thesis. They gave me lots of help and valuable suggestions about writing. I also thank Dr. Max Ramos, who helped me to set up the experiment and taught me how to use ABAQUS. I would like to thank all the staff in the master of engineering programme.

To my colleagues and friends at the DCRC, they made everyone feel like in a family here. To Alex, Prasika, Joe, Dr. Pui Ming, Yasser and Gijs, thank you for your help and advice.

Thank you, my best friends Yin Qi and Xiaojing Ma, you are always considerate and tender. To my friends in New Zealand, Zhipeng Xu, Yu Liu, Wenwen Zhao and Nasim, they really helped me to pass my hardest time in New Zealand.

Finally, I wish to give my most sincere appreciation to my family in China. They always stay behind me and support me. Thank you, Grandpa, Grandma, Dad Binqi Lan, Mum Wenxian Tong. Without their support and encouragement, the thesis would not have been possible. 


\begin{abstract}
During phonation, the vocal folds collision during the glottal closure is considered to be a risk factor for pathological development. This thesis is aimed at designing a dependable finite element analysis (FEA) model of the vocal folds for frequency and dynamic analysis and for calculating the impact stress between the vocal folds during glottal closure. A three-dimensional model with irregular geometry and a layered structure was designed. The measured viscoelastic properties of the vocal-fold mucosa and the transverse isotropic elastic properties of the vocal fold muscle are applied to the model. The boundary conditions are assumed to be fixed on lateral, anterior and posterior surfaces based on anatomical structure analysis. This model is symmetrical about the right and left vocal folds.
\end{abstract}

The frequency and dynamic characters are presented using the software ABAQUS. The FEA model is validated by both experimental modal analysis (EMA) model results and in-vivo experimental results from the literature.

In the vibration analysis, the eigenfrequency and eigenmode of the FEA model are determined. The model results compare well with the experiments performed on a silicone vocal fold model. The eigenmodes show the vibration direction at different excitation frequencies. In the closure process, the closure and collision dynamic results are obtained. The results show that: (1) the closure process is independent of the subglottal pressure; (2) the glottal opening amplitude and closing velocity vary approximately linear with the subglottal pressure; (3) the maximum impact stress occurs on the mid area of the inferior surfaces; (4) the impact stress is approximately linear with the subglottal pressure; and (5) the impact stress will cause vocal fold tissue damage when the subglottal pressure is over $800 \mathrm{~Pa}$.

It is anticipated that the model will help to identify voice disorders such as vocal-fold paralysis and vocal-fold nodules. 
ACKNOWLEDGEMENT.

.. $\mathbf{i}$

ABSTRACT ..........................................................................................................................

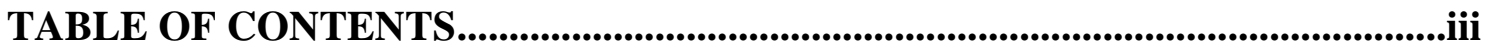

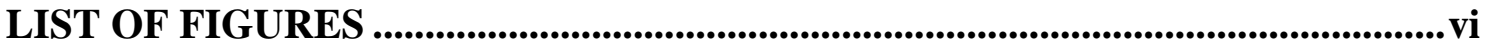

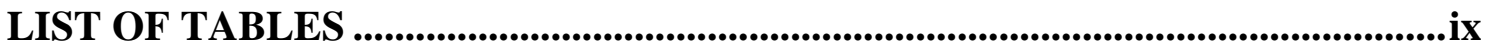

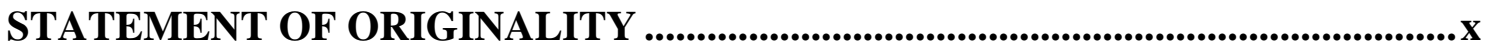

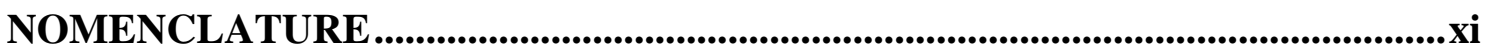

Chapter 1 INTRODUCTION............................................................................... 1

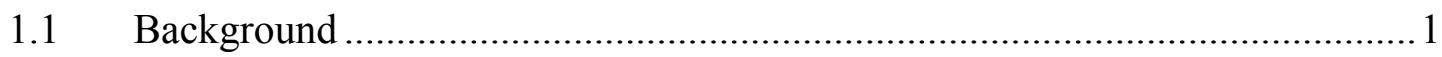

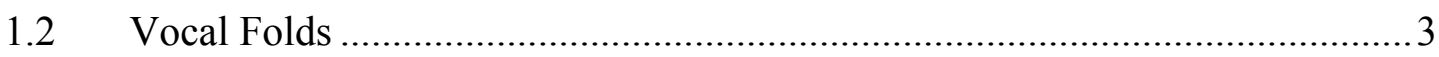

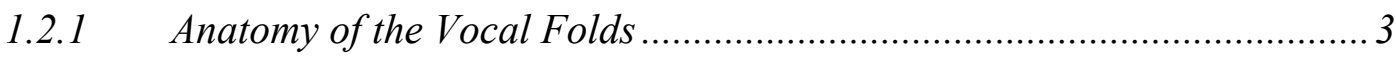

1.2.2 The Vocal Folds' Multilayer Structure ................................................... 5

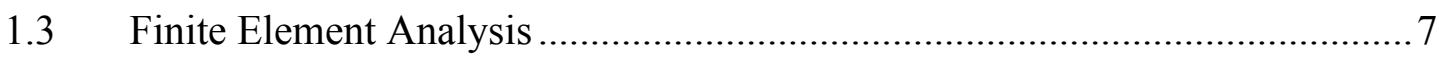

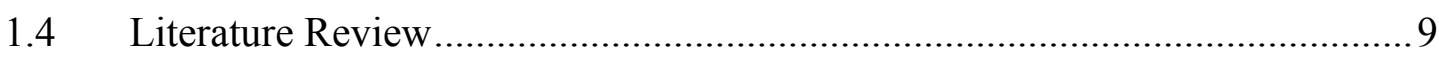

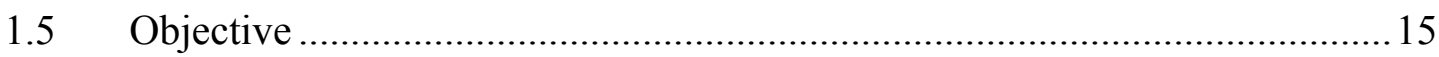

Chapter 2 FINITE ELEMENT MODEL ...................................................................17

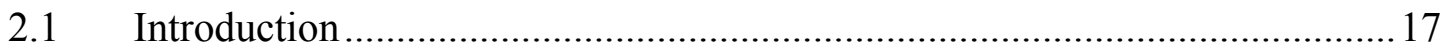

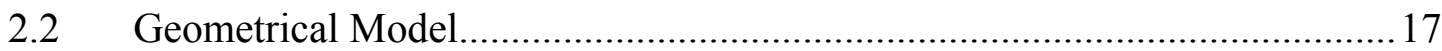

2.2.1 Mathematical Description of the Vocal Fold........................................ 17

2.2.2 Building the Model Using Solidworks ................................................... 19

2.2.3 Testing of Model Geometry in ABAQUS.............................................. 21

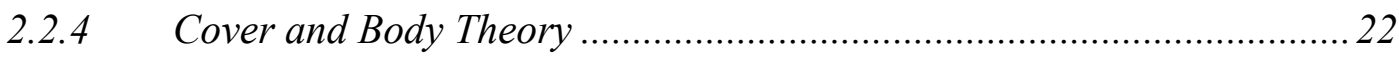

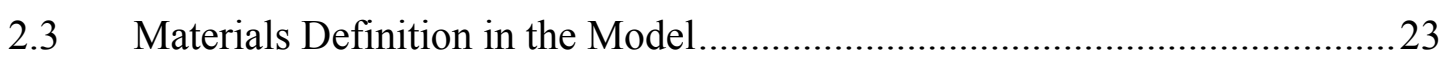

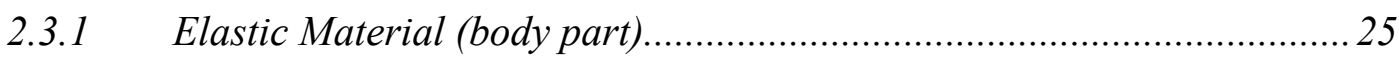

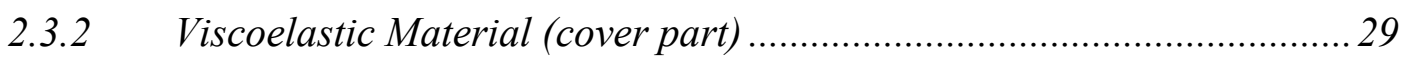

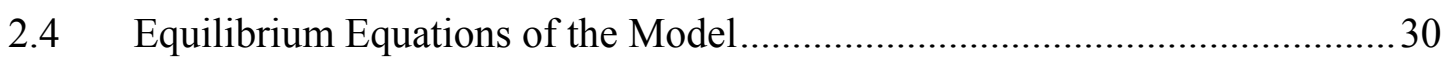

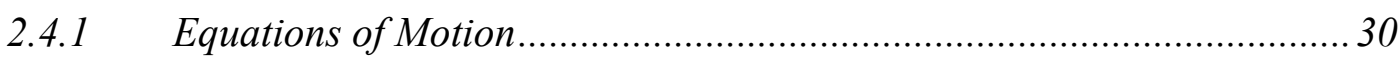

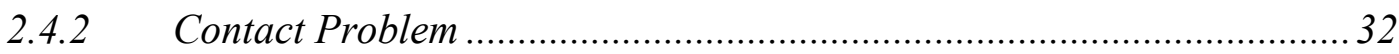

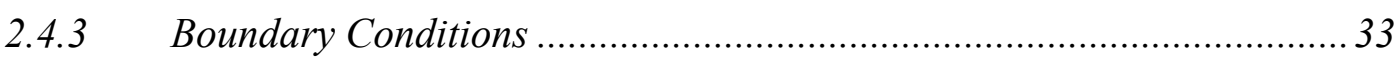

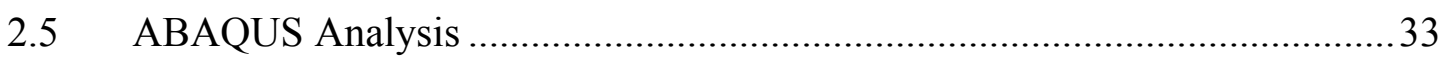


2.5.1 Build Geometrical Parts ......................................................................... 34

2.5.2 Define Material Properties .................................................................. 34

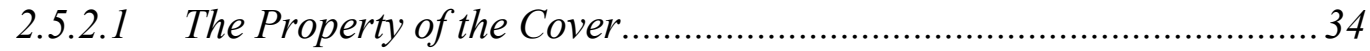

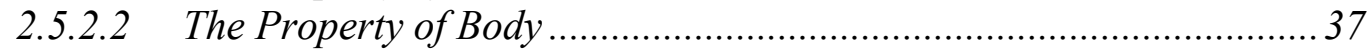

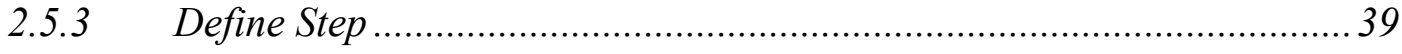

2.5.4 Assemble Model, Define Interaction, Load and Boundary Condition .... 40

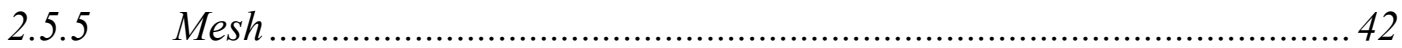

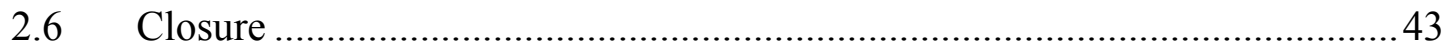

Chapter 3 EXPERIMENT INVESTIGATION ............................................................4

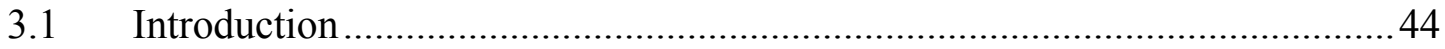

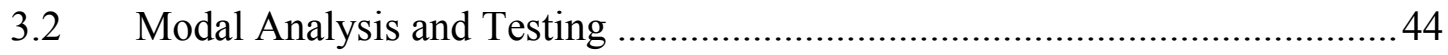

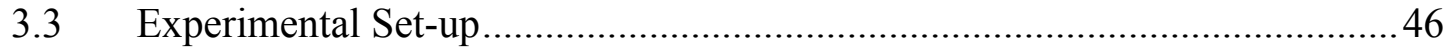

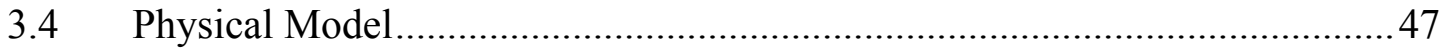

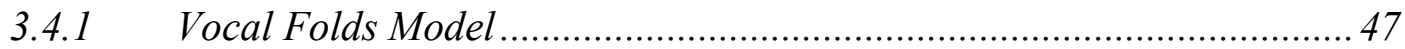

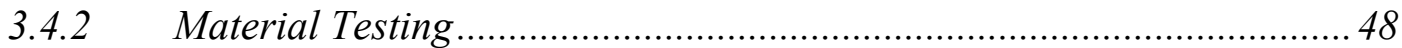

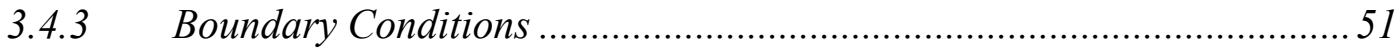

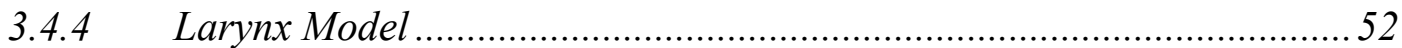

3.5 Experimental Equipment.....................................................................52

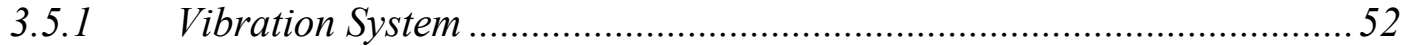

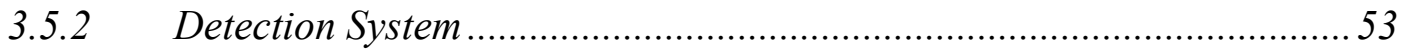

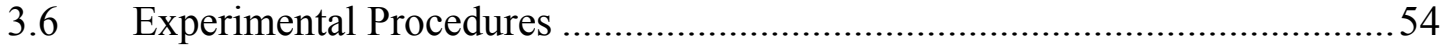

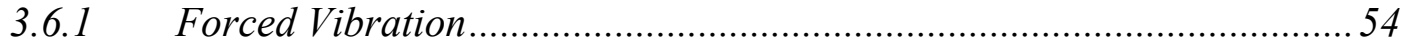

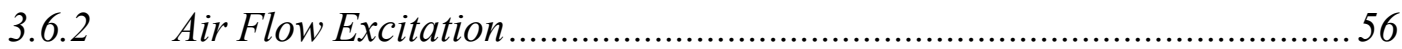

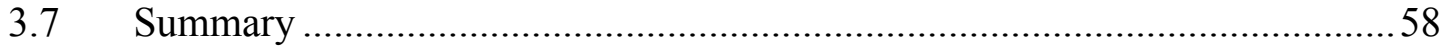

Chapter 4 RESULTS ..............................................................................................59

$4.1 \quad$ Introduction ...................................................................................... 59

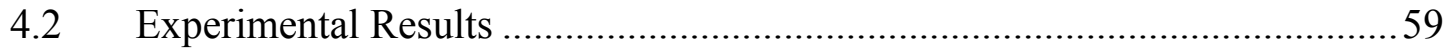

4.2.1 Forced Vibration Results ................................................................... 59

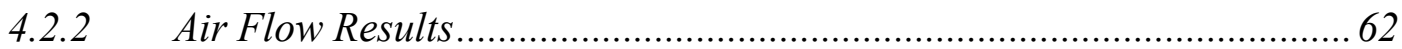

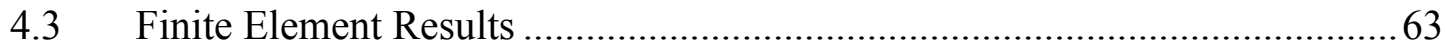

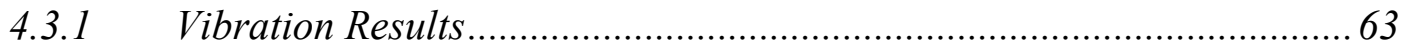

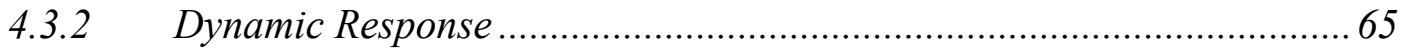

4.3.2.1 Glottal Closure Dynamics................................................................ 65

4.3.2.2 Glottal Collision Dynamics.............................................................. 69

Chapter 5 DISCUSSION ............................................................................................74

$5.1 \quad$ Introduction ........................................................................................ 


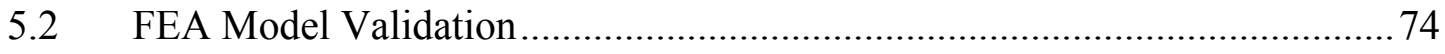

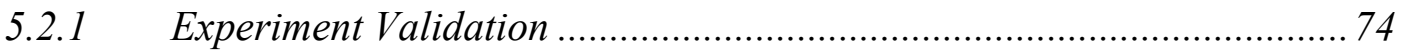

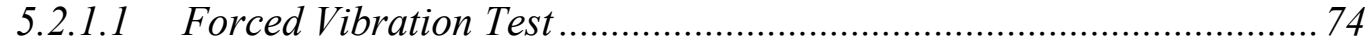

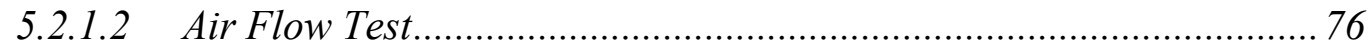

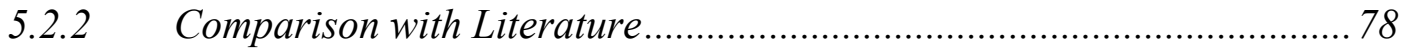

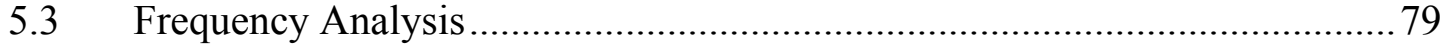

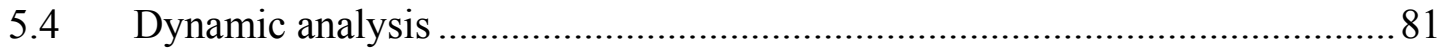

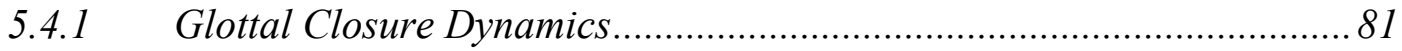

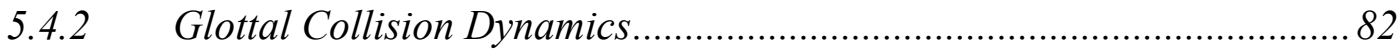

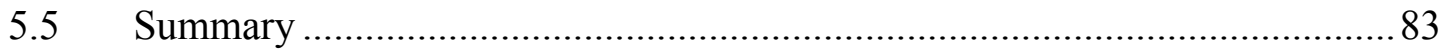

Chapter 6 CONCLUSIONS AND FUTURE WORK ................................................85

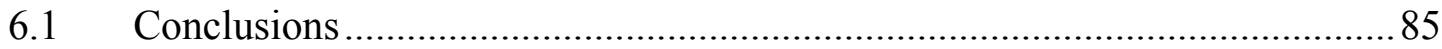

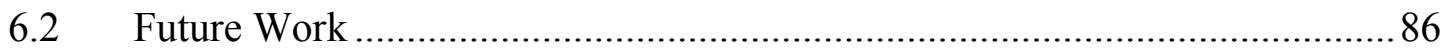

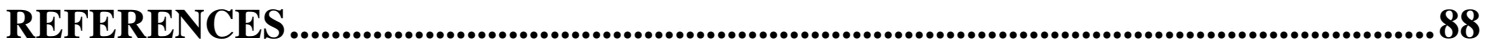

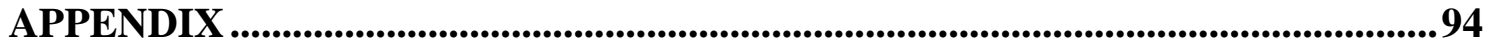

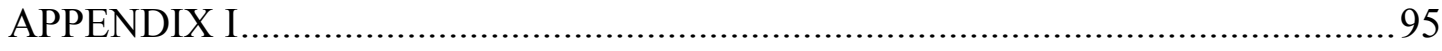

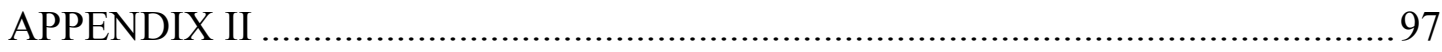

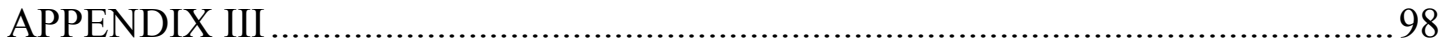




\section{LIST OF FIGURES}

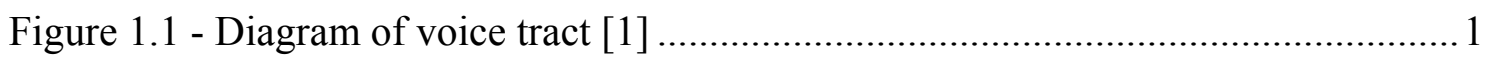

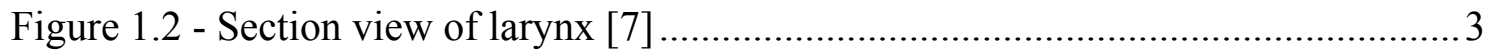

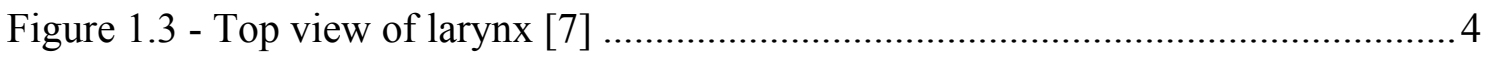

Figure 1.4 - Top view of larynx with muscles and cartilage exposed [2] ....................... 4

Figure 1.5 - Multilayered structure of the vocal fold's tissue.......................................... 5

Figure 1.6 - Different schemes used for describing the layered structure of the vocal

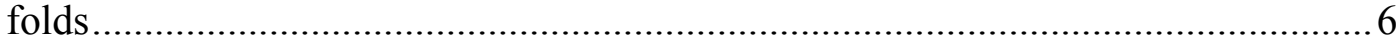

Figure 1.7 - Thickness of different layers[13] ........................................................ 7

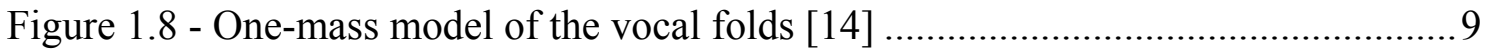

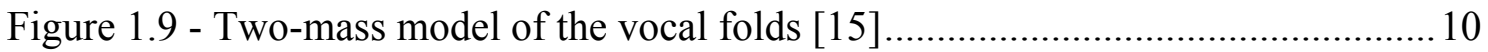

Figure 1.10 - Lumped-element representation of the body-cover structure of the vocal

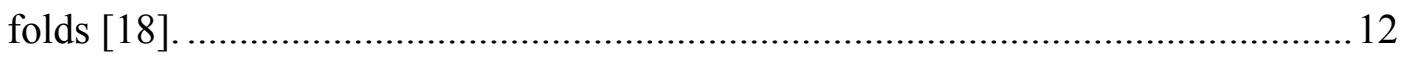

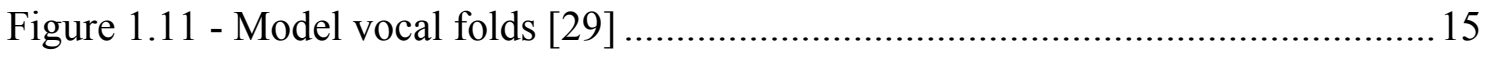

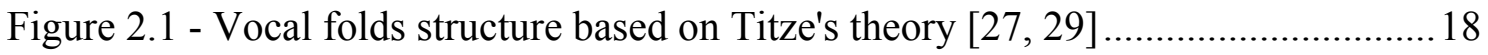

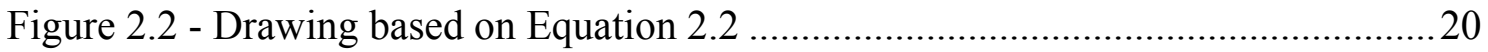

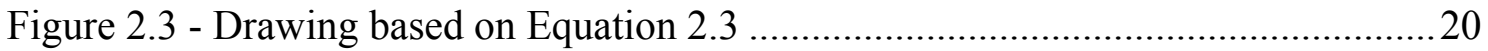

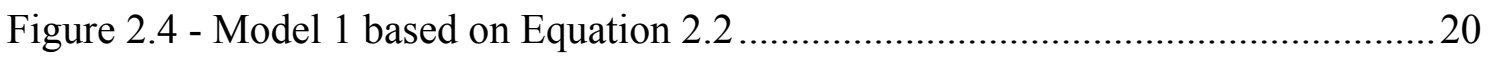

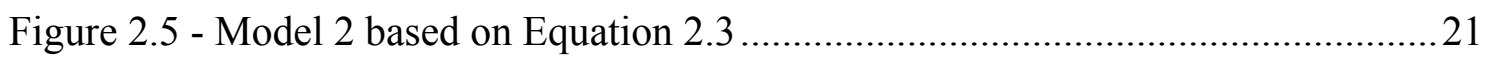

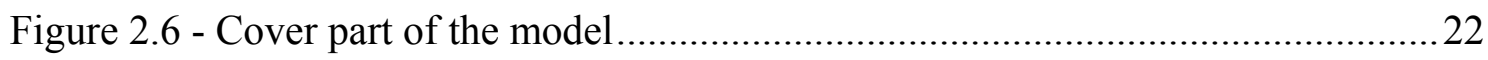

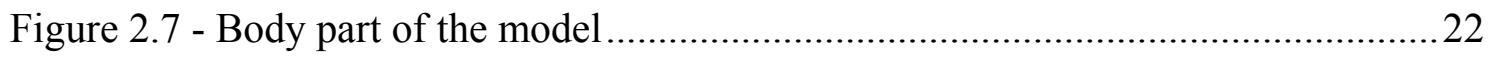

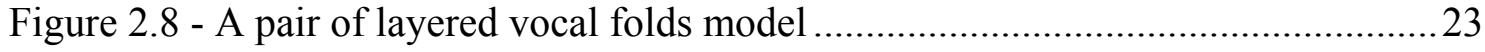

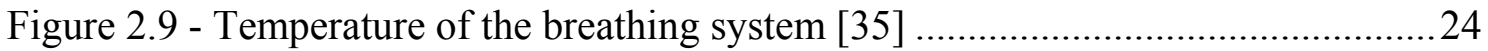

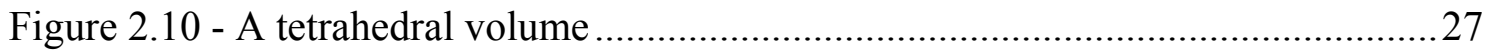

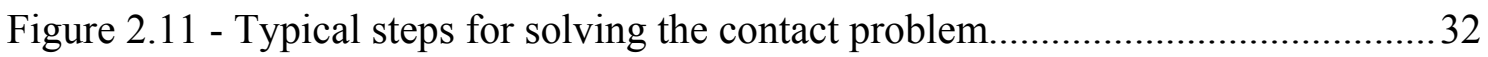

Figure 2.12 - Elastic shear modulus of the human vocal fold cover (59-year-old- male)

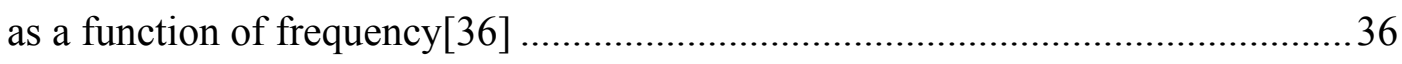

Figure 2.13 - Viscous shear modulus of the human vocal fold cover (59-year-old-male)

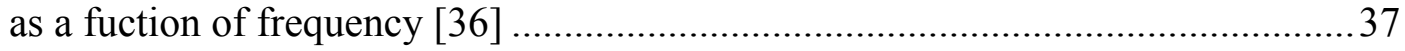

Figure 2.14 - Selected area in the middle of the vocal fold model ...............................40

Figure 2.15 - Distributed load (due to the subglottal pressure) on the inferior surface .. 41

Figure 2.16 - Assembled model with defined boundary conditions ..............................42 
Figure 2.17 - Meshed by tetrahedral element (in ABAQUS)

Figure 3.1 - (a) A block diagram of the forced vibration experimental set-up (b) A

block diagram of the second experimental set-up..............................................46

Figure 3.2 - The silicone model of the left side vocal fold 48

Figure 3.3 - Stress-strain curves with the vocal fold length of the lowest vibration

frequency as a reference. $\mathrm{M} 1 \mathrm{~L}=$ man $\# 1$, the left vocal fold [48]...... 48

Figure 3.4 - Stress-strain curves of a sample of vocal fold cover tissue stretched and released from high to low frequencies [39].

Figure 3.5 - HK505-S benchtop material testing machine 49

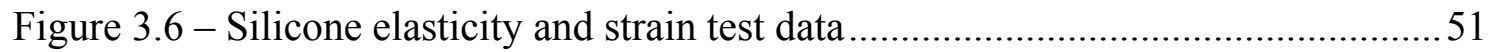

Figure 3.7 - The silicone vocal folds model fixed in the cartilage mould ......................52

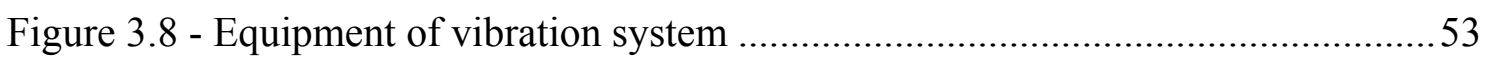

Figure 3.9 - Laser sensor head, vibrometer controller and oscilloscope.........................54

Figure 3.10 - Photo of the position of the shaker and the model ...................................55

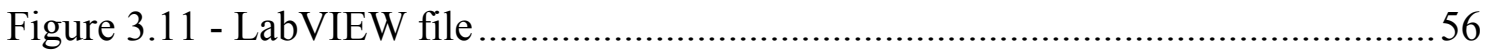

Figure 3.12 - Photo of the position of the laser head and larynx model .........................57

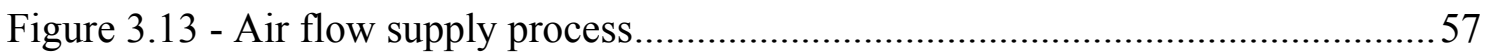

Figure 4.1 - Frequency response of the left vocal fold element.................................... 60

Figure 4.2 - Frequency response of the right vocal fold element..................................60

Figure 4.3 - Excited motion at frequencies 130, 140 and $150 \mathrm{~Hz}(\mathrm{left}) \ldots \ldots \ldots \ldots \ldots \ldots \ldots \ldots \ldots . . . . . . . . . . . .61$

Figure 4.4 - Excited motion at frequencies of 110, 120 and $130 \mathrm{~Hz}$ (left) ....................61

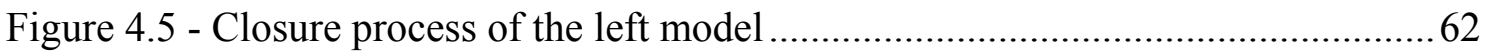

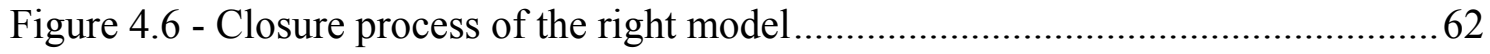

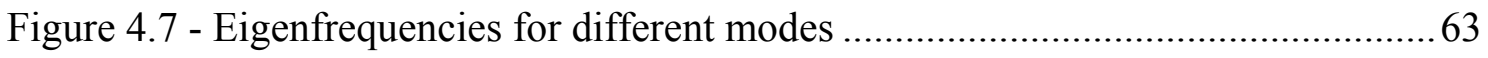

Figure 4.8 - Vocal fold eigenmodes at (a) $125.07 \mathrm{~Hz}$, fundamental frequency (b) 184.88

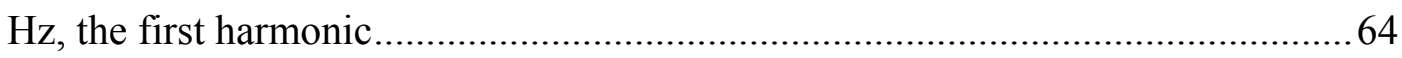

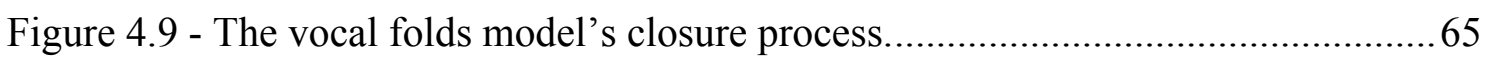

Figure 4.10 - Selected nodes for glottal opening test................................................66

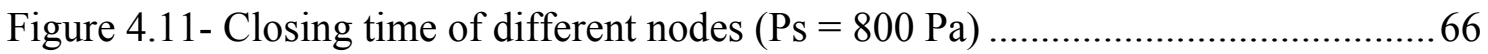

Figure 4.12 - Closing velocity of different nodes on inferior edge of the right vocal fold.

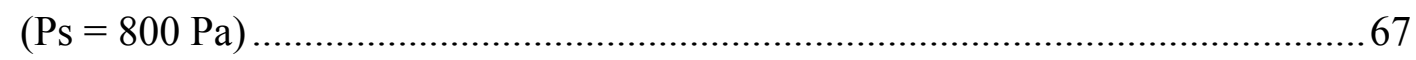

Figure 4.13 - Effect of the subglottal pressure on the vocal fold movement. .................68

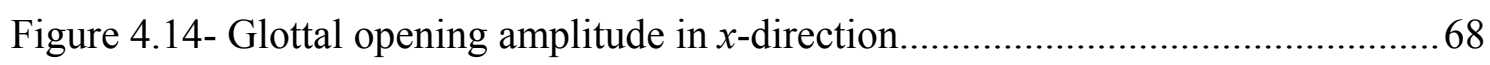

Figure 4.15 - Node 250 closing velocity under various subglottal pressures .................69

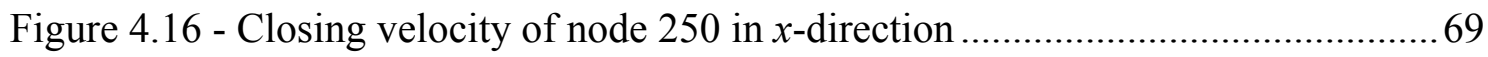


Figure 4.17 - Total contact normal force under various subglottal pressures ( $\mathrm{Ps}=600$,

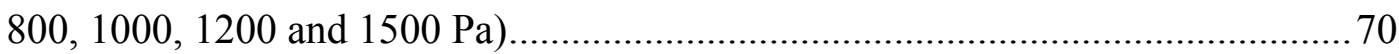

Figure 4.18 - Total contact area under various subglottal pressures $(\mathrm{Ps}=600,800,1000$, 1200 and $1500 \mathrm{~Pa})$ 70

Figure 4.19 - Comparison of the max contact force between selected points and the whole model points $(\mathrm{Ps}=800 \mathrm{~Pa})$ 71

Figure 4.20 - Maximum contact stress of the selected points during closure $(\mathrm{Ps}=800 \mathrm{~Pa})$

Figure 4.21 - The highlight points present the maximum contact normal force and contact stress during glottal pressure. 72

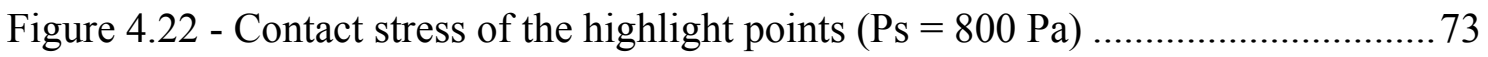

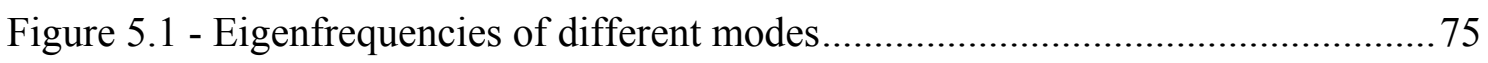

Figure 5.2 - The detected point will change if the object bends. .................................. 77

Figure 5.3 - Closure process of the right model........................................................ 78

Figure 5.4 Eigenmodes (a) one (at $81.87 \mathrm{~Hz}$ ) (b) two (at $127.72 \mathrm{~Hz}$ ) .......................... 79 


\section{LIST OF TABLES}

Table 2-1 - Natural frequencies of model 1 and model 2 ...........................................2 21

Table 5-1 Comparison of theoretical results and experimental results ..........................76

Table 5-2 Comparison of theoretical results and in-vivo experimental results ..............79 


\section{STATEMENT OF ORIGINALITY}

'I hereby declare that this submission is my own work and that, to the best of my knowledge and belief, it contains no material previously published or written by another person nor material which to a substantial extent has been accepted for the qualification

of any other degree or diploma of a university or other institution of higher learning, except where due acknowledgment is made in the acknowledgments.'

(signed)

(date) 
Symbol

d

E

$E^{\prime}$

$E_{b}$

$E_{c}$

$e_{0}$

$\varepsilon$

F

$F_{1}$

$F_{2}$

G

$g$

$G^{\prime}$

$G^{\prime \prime}$

$G_{s}(\omega)$

$G_{l}(\omega)$

$G_{\infty}$

$h$

K

$k$

$k_{b}$

$k_{c}$

$k_{l}$

$k_{u}$

$\lambda_{m}$

$L$

M

\section{Meaning}

The constant of the damper

The depth of the vocal folds

The thickness of the cover

The Young's modulus in the transverse plane

The Young's modulus along the y axis

Body tissue's Young's modulus

Young's modulus of the cover

Constant strain

Strain tensor

The collision force

Forces acting on $M_{1}$

Forces acting on $M_{2}$

The gap normal to the contact surface

The coefficient that describes the nonlinearity of the spring

The elastic shear modulus

The viscous shear modulus

The storage modulus

The loss modulus

The long-term shear modulus

The vertical distance below the superior surface

The constant of the spring

The non-linear stiffness

the stiffness of the body tissue

Mass connecter spring

the stiffness between the cover and body

the stiffness of the cover tissue and.

Relaxation times

the length of vocal folds

The weight of mass 


$\begin{array}{ll}M_{1} & \text { Mass of block one } \\ M_{2} & \text { Mass of block two } \\ \eta & \text { The unit normal vector of the contact surface } \\ P s & \text { The dynamic viscosity } \\ \rho & \text { Subglottal pressure } \\ \sigma & \text { Poisson's ratio } \\ s & \text { Stress } \\ T & \text { A shaping factor } \\ U & \text { The thickness of the vocal folds } \\ \mu & \text { The strain energy } \\ \mu^{\prime} & \text { The shear modulus in the transverse plane } \\ V & \text { The shear modulus along the y axis } \\ v & \text { The volume of the element } \\ v^{\prime} & \text { The Poisson's ratio in the transverse plane } \\ W_{p} & \text { The Poisson's ratio along the y axis } \\ w & \text { The virtual work } \\ y & \text { The angular deviation of the superior-medial edge with respect to } \\ & \text { the vertical mid-plane of the glottis. }\end{array}$




\section{Chapter 1 INTRODUCTION}

\subsection{Background}

Voice is essential to most of people in their personal and professional lives. From birth, human beings use their voice as the most common way to communicate with others. In the social life, people talk to others to deliver their message to, sing a song to show their emotions, or talk in talk show to entertain the audience.

The human's voice comes from the vocal organ. As shown in Figure 1.1, the human's vocal organ includes three voice subsystems. These are the air pressure, the vibratory and the resonating systems [1].

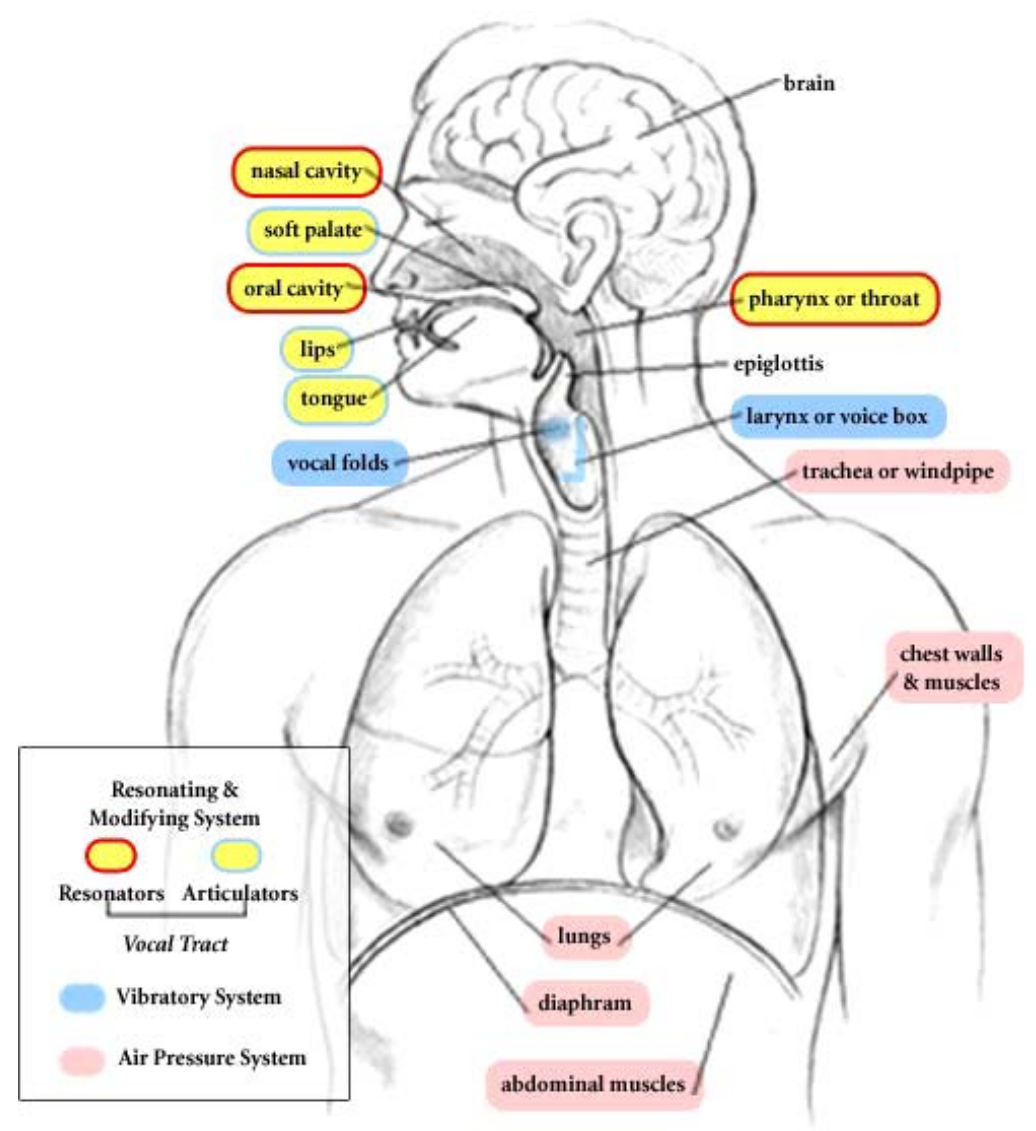

Figure 1.1 - Diagram of voice tract [1] 
Air pressure system produces voice starting with airflow from the lungs, which is coordinated by the action of the diaphragm, abdominal and chest muscles. Vibratory system consists of a larynx and the vocal folds which are the vibratory system of the vocal organ. Resonating system is the organ in which people produce recognizable sound and form their personal quality of voice.

Of various elements of these three systems, the vocal folds in the larynx are the most important for phonation. During phonation, the air pressure system provides and regulates air pressure to the larynx which causes the vocal folds to vibrate. The vocal folds abduction (open) and adduction (close) controls the airflow through the larynx. The basic sound produced by the vibration of the vocal folds is called "voiced sound". Voiced sound is amplified and modified by the resonating system which consists of vocal tract resonators (the throat, mouth cavity, and nasal passages) and the vocal tract articulators (the tongue, soft palate, and lips). The first part produces a person's recognizable voice. The second part which modifies the voiced sound is responsible for the formation of recognizable words [2]. A human's sound is in a sequence of vibrator cycles with a speed from about 100 to $300 \mathrm{~Hz}$ [3]. A higher voice is produced by increasing the frequency of the vocal folds vibration and a louder voice is formed by increasing the amplitude of the vocal folds vibration.

During phonation, the vocal folds will touch each other at the end of each cycle. The collision between the vocal folds is associated with aerodynamic factors that are linked to voice quality [4]. Furthermore, the increased stress on the surfaces during collision is considered as an important reason for tissue damage [5]. It is commonly believed that vocal nodules which are benign growths on the vocal folds result from repeated collision of the vocal folds during phonation and the strain component is relevant to the assessment of the impact of vocal fold collision on potential tissue damage. Vocal nodules will cause voice disorders, such as hoarseness, breathiness, harshness and so on. Clinical observations suggest that people with higher-pitched (higher frequency) voices and louder (higher amplitude) voices tend to be more susceptible to vocal nodules [6]. Thus, it is assumed that both the frequency and amplitude of the vocal folds' vibration play important roles in the vocal folds damage.

The vocal folds' biomechanical tissue characteristic is one of the primary factors in voice production. As it is impossible to do experiments on human beings and even hard 
to observe the physical movement details during phonation, a theoretical model will be an excellent tool to describe the process of phonation.

One of the aims of this research is to obtain a realistic value of the impact stress during vocal folds collision. Finite element analysis (FEA), which gives the possibility of accurate detail geometry and material properties in a model, is used in this work.

\subsection{Vocal Folds}

The vocal folds' shape and mechanical properties determine their frequency characteristics like frequency and dynamic amplitude. Therefore, for the purposes of this research, it is important to fully understand the geometrical and mechanical details of the vocal folds in order to set up a dependable model.

\subsubsection{Anatomy of the Vocal Folds}

Vocal folds used to be called vocal cords because they were thought to be vibrating like strings on a guitar. Careful anatomical examination has shown this to be incorrect.

Figure 1.2 and Figure 1.3 show the position of the vocal folds in the larynx. The vocal folds are formed from ligament-like tissue located in the middle of the larynx and fixed on arytenoid cartilages and the thyroid cartilage. They are stretched horizontally across the larynx from front to back. When they are open, the glottis is a V-shape, with the wide part of the $\mathrm{V}$ at the back and the point of the $\mathrm{V}$ at the front.

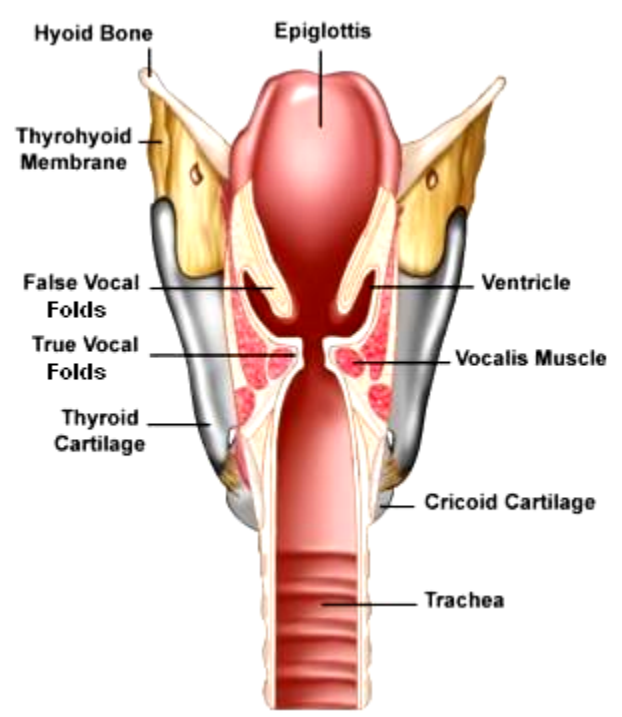

Figure 1.2 - Section view of larynx [7] 

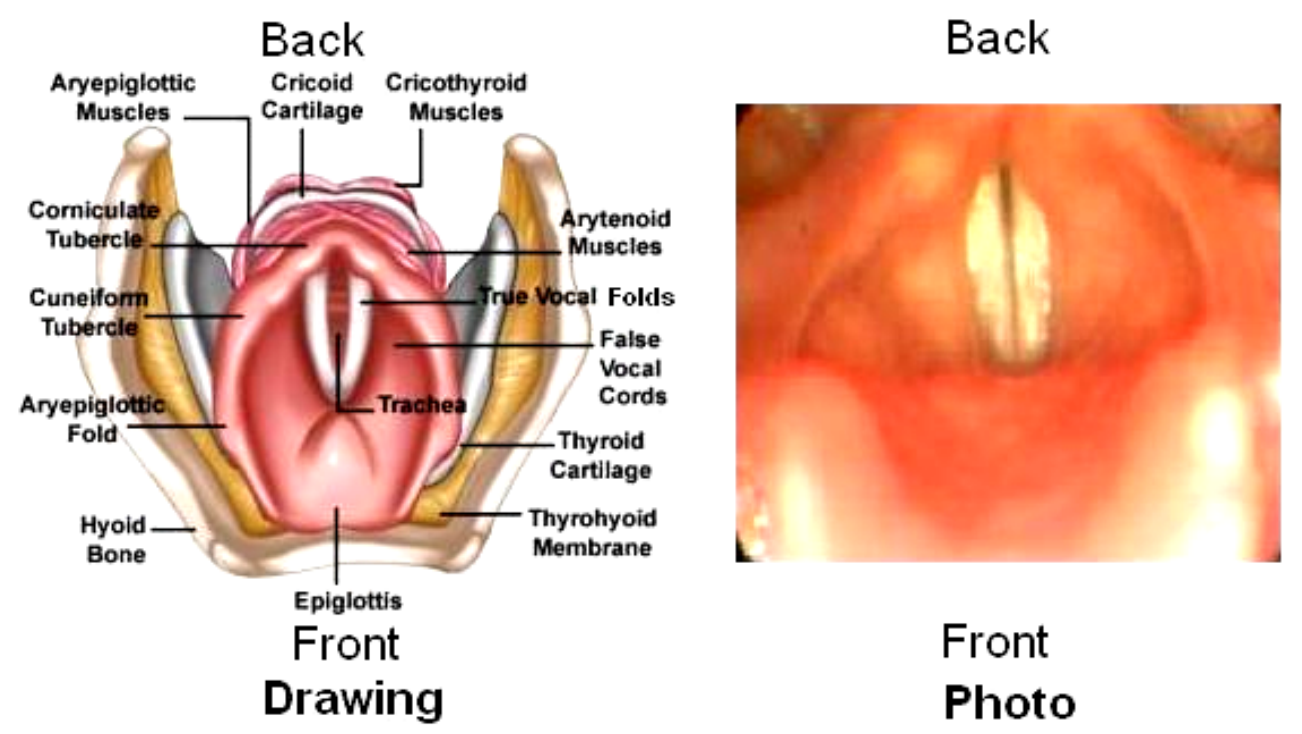

Figure 1.3 - Top view of larynx [7]

The muscles in the larynx control the adduction and abduction of the vocal folds. The muscles and cartilages shown in Figure 1.4 control the movement of the vocal folds. Posterior cricoarytenoid muscles are the only muscles which control abduction. In breathing process, the glottis opens when posterior cricoarytenoid muscles contract, and pull the back ends of the arytenoid cartilages up. This pulls the front ends apart which attach the vocal folds. The other muscles which relate to the vocal folds control adduction. Their contraction will change the vocal folds' properties such as length, tension and thickness, which will affect the voice's frequency and amplitude.

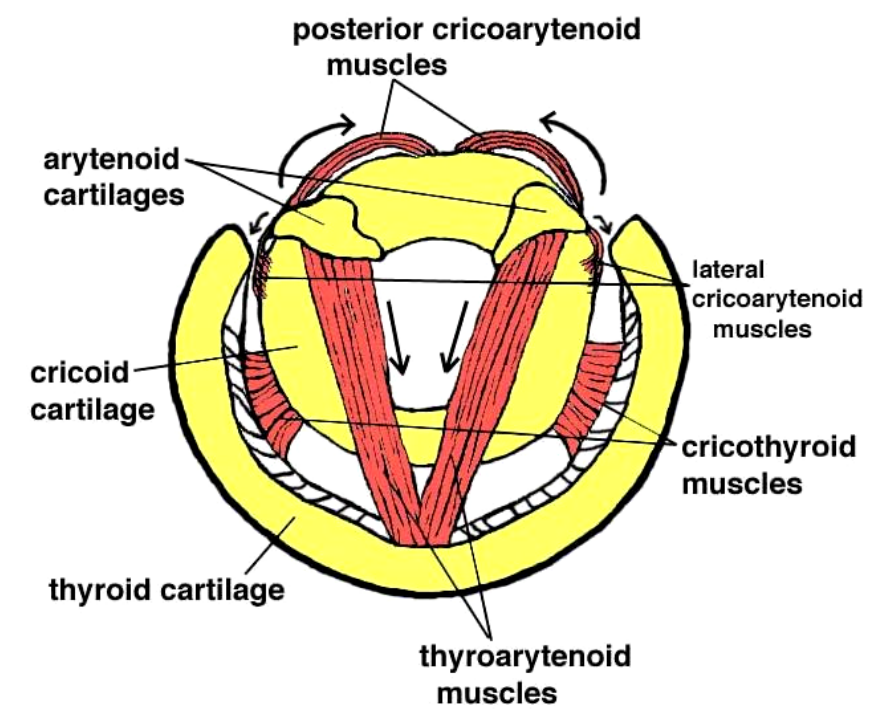

Figure 1.4 - Top view of larynx with muscles and cartilage exposed [2] 


\subsubsection{The Vocal Folds' Multilayer Structure}

The vocal fold's structure is multilayer. Figure 1.5 [8] shows a drawing of the layered structure of the right vocal fold. The outermost layer is named the epithelium which is made of stratified squamous tissue of $0.05-0.1 \mathrm{~mm}$ thick [9]. The epithelium encapsulates softer, fluidlike tissue, somewhat like a balloon filled with water [10]. Between the epithelium and muscle, a layered system which is called lamina propria (LP) consists of nonmuscular tissues. The whole LP is made up of collagens and elastins. However, various types of collagens and elastins are found in the LP of the human vocal folds. The three types are elaunin, oxytalan and elastin fibres. Oxytalan is composed of microfibrils 10 to $12 \mathrm{~nm}$ in diameter. Elaunin has these microfibrils and a small amorphous component. Elastin fibres have a larger amorphous component as a core, with the microfibrils surrounding the core [8]. The LP consists of three layers: the superficial layer, the middle or intermediate layer and the deep layer. The superficial layer of the LP consists primarily of loosely organized elastin fibres surrounded by interstitial fluids and about $0.5 \mathrm{~mm}$ thick. The intermediate layer is made up primarily of oxytalan, whose fibres are more uniformly oriented in the anterior-posterior direction. The deep layer is made up primarily of elastin fibres that are like a cotton thread which is nearly inextensible and run parallel along the anterior-posterior direction.

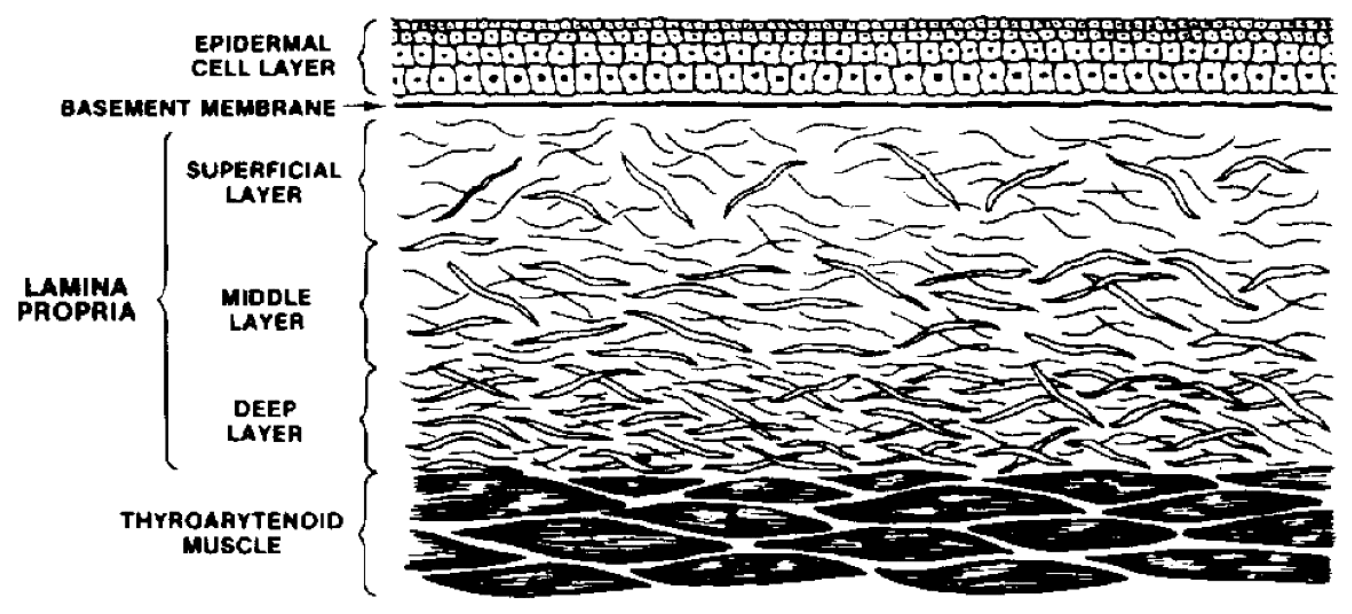

Figure 1.5 - Multilayered structure of the vocal fold's tissue

Figure 1.5 shows the summary of the different simplified schemes used to describe the structure of the vocal folds, namely the theory of Hirano [11] (a three-layer scheme) and the cover-body theory (a two-layer scheme). 


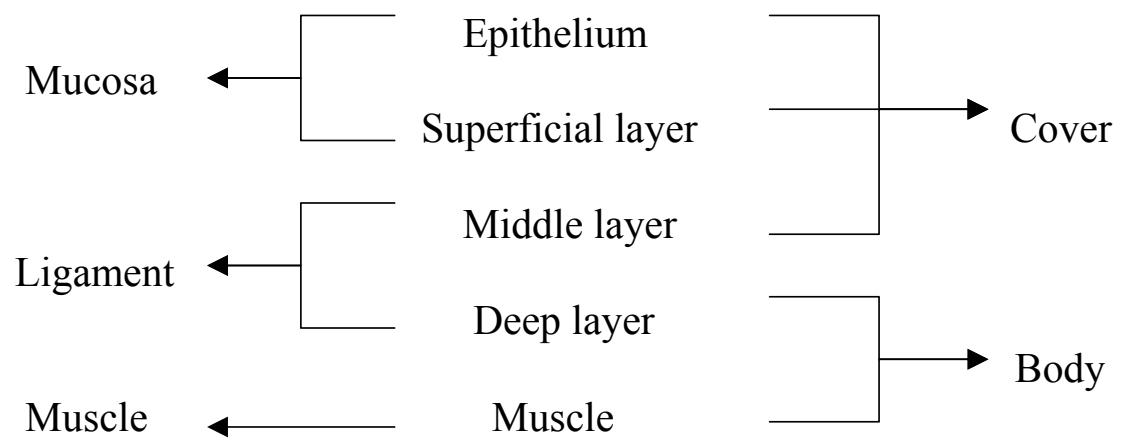

Figure 1.6 - Different schemes used for describing the layered structure of the vocal folds

The three-layer scheme is the most popular theory presented by Hirano [11] who differentiates between various layers on a relative basis. The outer tissue layer is called the "mucosa." It is a combination of the epithelium (mucus membrane) and the superficial mucus layer in Hirano's terminology. Its thickness is $0.5 \mathrm{~mm}$ at the posterior, and tapers to half of this value towards the anterior. The next layer, a combination of what Hirano has termed the intermediate and deep transition layers of the lamina propria, will be given the more common term "ligament". It is $1 \mathrm{~mm}$ thick at the posterior and also tapers linearly to half of that value at the anterior portion. The remainder is muscle.

The cover-body theory is founded on relating the properties of different layers in the vocal folds [12]. As shown above, the superficial layer and the middle layer are made of loosely organized microfibrils and small components, and the epithelium consists of softer, fluidlike tissue. These three layers show similar mechanical properties of viscoelasticity and isotropicity. The deep layer and the muscle present the properties of elasticity and direction dependence. Therefore, the studies of the vocal folds suggest that epithelium, lamina propria's superficial and middle layers could be effectively represented together as the cover with the deep layer and muscle represented as the body [13]. The general thickness of the cover is $1 \mathrm{~mm}$ at posterior portion and tapers linearly to half of this value at anteriority. Figure 1.7 shows the thickness of different layers. 


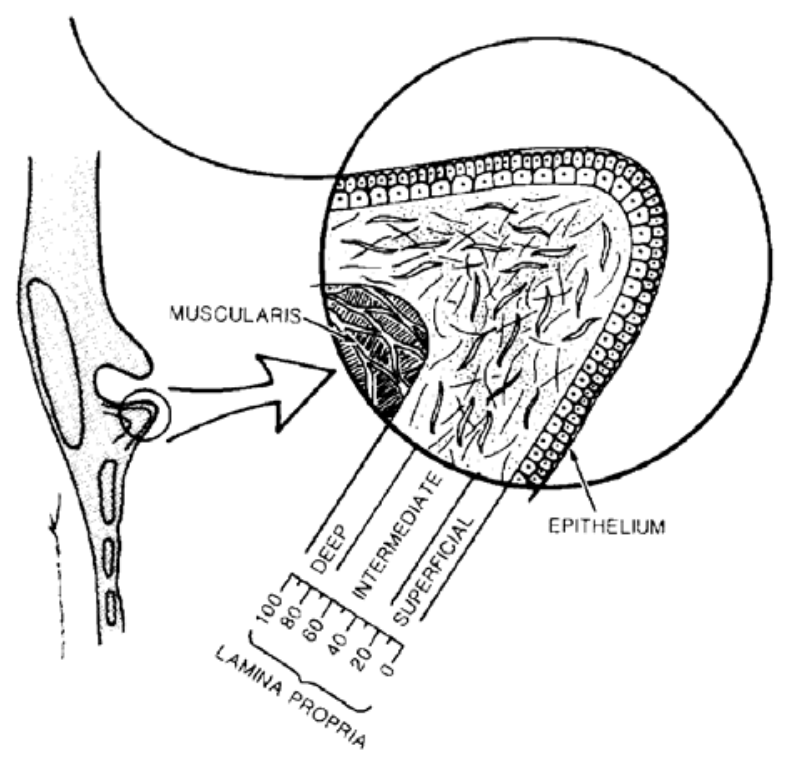

Figure 1.7 - Thickness of different layers[13]

\subsection{Finite Element Analysis}

A theoretical model of the vocal folds could be an excellent tool to investigate their frequency characteristics and dynamic response during phonation. However, the geometry of the vocal folds is difficult to describe due to the layered structure and the curved surface, making the model hard to solve manually. Finite element analysis (FEA) could supply a more accurate solution and provide excellent visual aids. A combination of 3D design software and finite element analysis software are used.

The finite element method is a numerical procedure which can model the behaviour of a structure with great accuracy. It can deal with complicated one-, two- and threedimensional geometry, variations in material properties, and various structural restraints.

Following the general FEA procedure, there are six steps in the model's creation:

(1) Definition of dynamic finite element equations;

(2) Identification of the target geometry (anatomical properties);

(3) Determination of constitutive equations and material properties (e.g., tissue elasticity, density, and incompressibility);

(4) Specification of the boundary conditions and constraints; 
(5) Implementation of the finite elements (discrimination of the domain and assembly of element equations);

(6) Solution of the model with numerical results. Quantities such as fibre length, area, and orientation were classified as anatomical properties, while passive and contractile characteristics of the intrinsic fibres were classified as material properties.

To define the target geometry, Solidworks ${ }^{\mathrm{TM}}$ could be used. Solidworks ${ }^{\mathrm{TM}}$ 3D Mechanical Design Software is used as the standard 3D mechanical design program. It is particularly well suited for three-dimension perspective structure. It offers unmatched performance and value, leadership in innovation, and the largest community of users, allowing people to get product design work done quickly and accurately.

To solve the system governing equations and find the characteristics and response, particular software may be used. ABAQUS (HKS Inc.; Pawtucket, RI) is one of the best FEA programs currently available worldwide. It provides finite element solution techniques to simulate a wide variety of nonlinear, transient dynamics and quasi-static events in an accurate, robust, and efficient manner. ABAQUS has four analysis products: ABAQUS/Standard, ABAQUS/Explicit ABAQUS/Aqua, and ABAQUS/Design. ABAQUS/Explicit is a special-purpose analysis product that uses an explicit dynamic finite element formulation. It is suitable for modelling brief, transient dynamic events, such as impact and blast problems, and is also very efficient for highly nonlinear problems. The focus of this research is on calculating the impact stress between two vocal folds during phonation. The impact stress on vocal fold tissue is considered as one of the most important factors for the etiology of vocal nodules. The impact force is a type of force in physics which describes the effect that time has on accelerating bodies. It usually takes place in a very short time period of about 0.01 second. The model's geometry and material properties are nonlinear; therefore the problem is nonlinear too. This requires that the FEA software which calculates the deformation and force is good at solving nonlinear analysis. Therefore, ABAQUS/Explicit is appropriate software for this research. 


\subsection{Literature Review}

The idea of using a theoretical model or experimental model to simulate the vocal folds is not new. There are three kinds of models used in the literature: mass-spring-damper system model, FEA model and silicone model.

The mass-spring-damper model of the vocal folds was built by Flanagan and Landgraf in 1968 [14] as shown in Figure 1.8. In this model, the mass represents the vocal-fold length, the area of intraglottal surface and the vocal-fold weight. The spring and damper represent the viscoelastic property of the vocal fold tissue. The system is excited by a force $F(t)$, given by the subglottal air pressure $P(s)$ on the intraglottal surface. The force acts on the surface of the vocal folds as shown in Figure 1.8 (a).
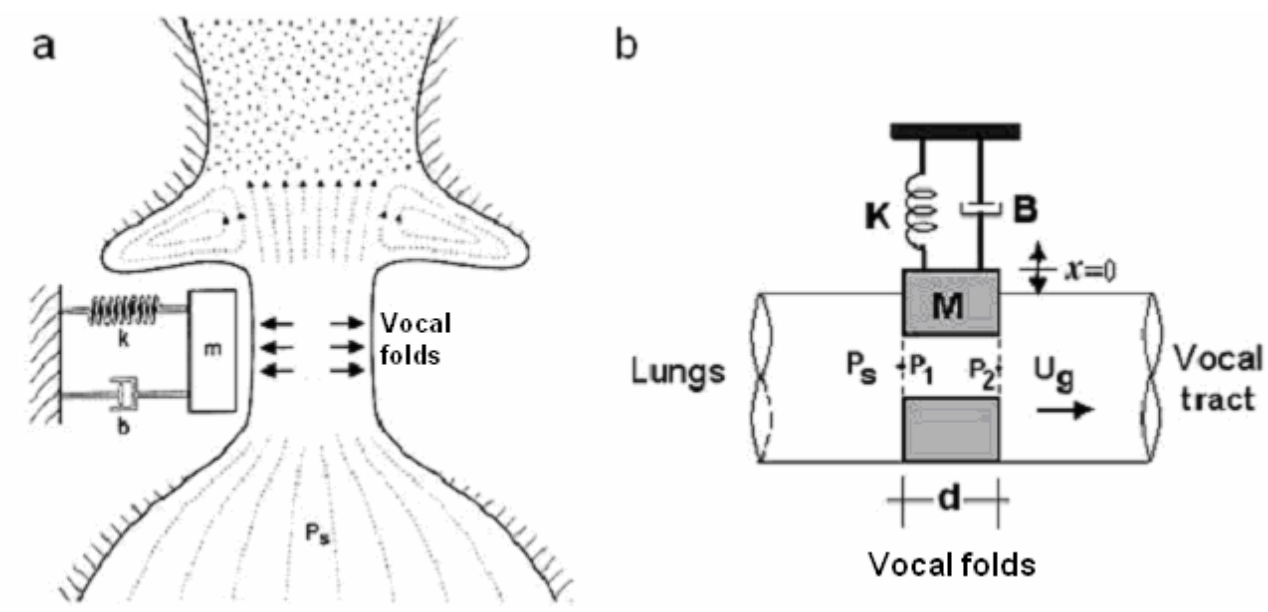

Figure 1.8 - One-mass model of the vocal folds [14]

The equation of this model is

$$
M x^{\prime \prime}(t)+B x^{\prime}(t)+K x(t)=F(t)
$$

where

$x(t)=$ Displacement of mass $\mathrm{M}$

$\mathrm{M}=$ The weight of mass

$\mathrm{K}=$ The constant of the spring

$\mathrm{B}=$ The constant of the damper

The one-mass model provides the simplest mechanical model of the vocal folds and produces just an acceptable synthesis of voice sounds and acoustic wave. However, it is a very simple model of one degree of freedom which describes an idealised case of opening and closing movement during phonation. 
A more completed model which could simulate the physiological details in the behaviour of the vocal folds is needed. Multiple-mass representations of the vocal folds were considered. In 1972, Ishizaka and Flanagan [15] built a simple and efficient mathematical model (IF72) by connecting two masses with springs and dampers as shown in Figure 1.9.

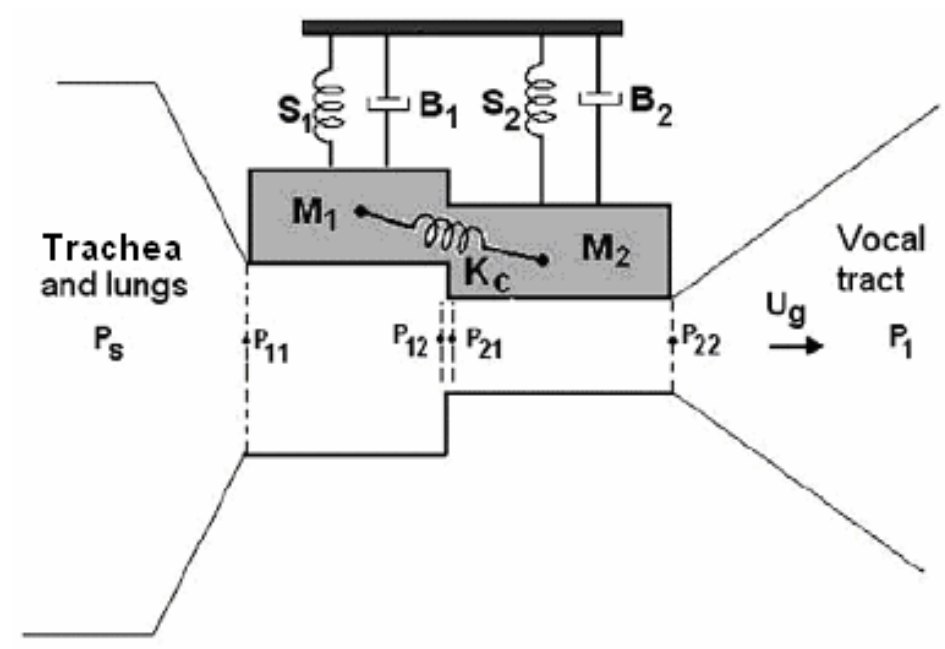

Figure 1.9 - Two-mass model of the vocal folds [15]

The model is a system with two degrees of freedom which could produce longitudinal and vertical phase differences. Each vocal fold consists of two mass-spring-damper oscillators. The masses are coupled through a third spring $k_{c}$. The model is assumed to be symmetrical: both sides of the vocal folds consist of the same components. For the accurate simulation of the elastic properties of the fold, the springs $S_{1}$ and $S_{2}$ are nonlinear, which represent the tension in the vocal folds. The nonlinear relation between the deflection from the position of equilibrium and the force required to produce this deflection is given by Equation 1.2, where $f$ is the force required to produce a deflexion $x, k$ is the non-linear stiffness and $g$ is the coefficient that describes the nonlinearity of the spring S. The mass connecter spring $k_{c}$ is linear. The viscous forces are modeled as linear damping terms, with $B_{1}$ and $B_{2}$ respectively.

$$
f=k x\left(1+g x^{2}\right)
$$

The equations that describe the dynamics of the system (the vocal folds) are given by 


$$
\left\{\begin{array}{c}
M_{1} x_{1}^{\prime \prime}+S_{1}\left(x_{1}\right)+B_{1}\left(x_{1}^{\prime}\right)+k_{c}\left(x_{1}-x_{2}\right)=F_{1} \\
M_{2} x_{2}^{\prime \prime}+S_{2}\left(x_{2}\right)+B_{2}\left(x_{2}^{\prime}\right)+k_{c}\left(x_{2}-x_{1}\right)=F_{2}
\end{array}\right.
$$

where $F_{1}$ and $F_{2}$ are forces acting on $M_{1}$ and $M_{2}$ over their displacements $x_{1}$ and $x_{2}$, given in terms of mean pressures acting on the vocal folds exposed faces and the subglottal pressure Ps.

The success of IF72 led to more accurate lumped mass systems. As many as 16 masses $[16,17]$ were used which were predicted to lead to a better understanding of the aerodynamic and produce a more natural sounding artificial voice. The classical IF72 model is essentially a "cover" model rather than the entire vocal fold model.

In 1995, Story and Titze [18] built a three-mass model (ST95) to simulate the body and cover structure as shown in Figure 1.10. This model is essentially based on IF72 twomass model. The cover portion of the vocal fold is divided into two equally thick elements. A third larger mass is added to simulate the body layer of the vocal fold. The springs $k_{u}$ and $k_{l}$ are used to represent the stiffness of the cover tissue and the stiffness between the cover and body. Spring $k_{b}$ represents the stiffness of the body tissue.

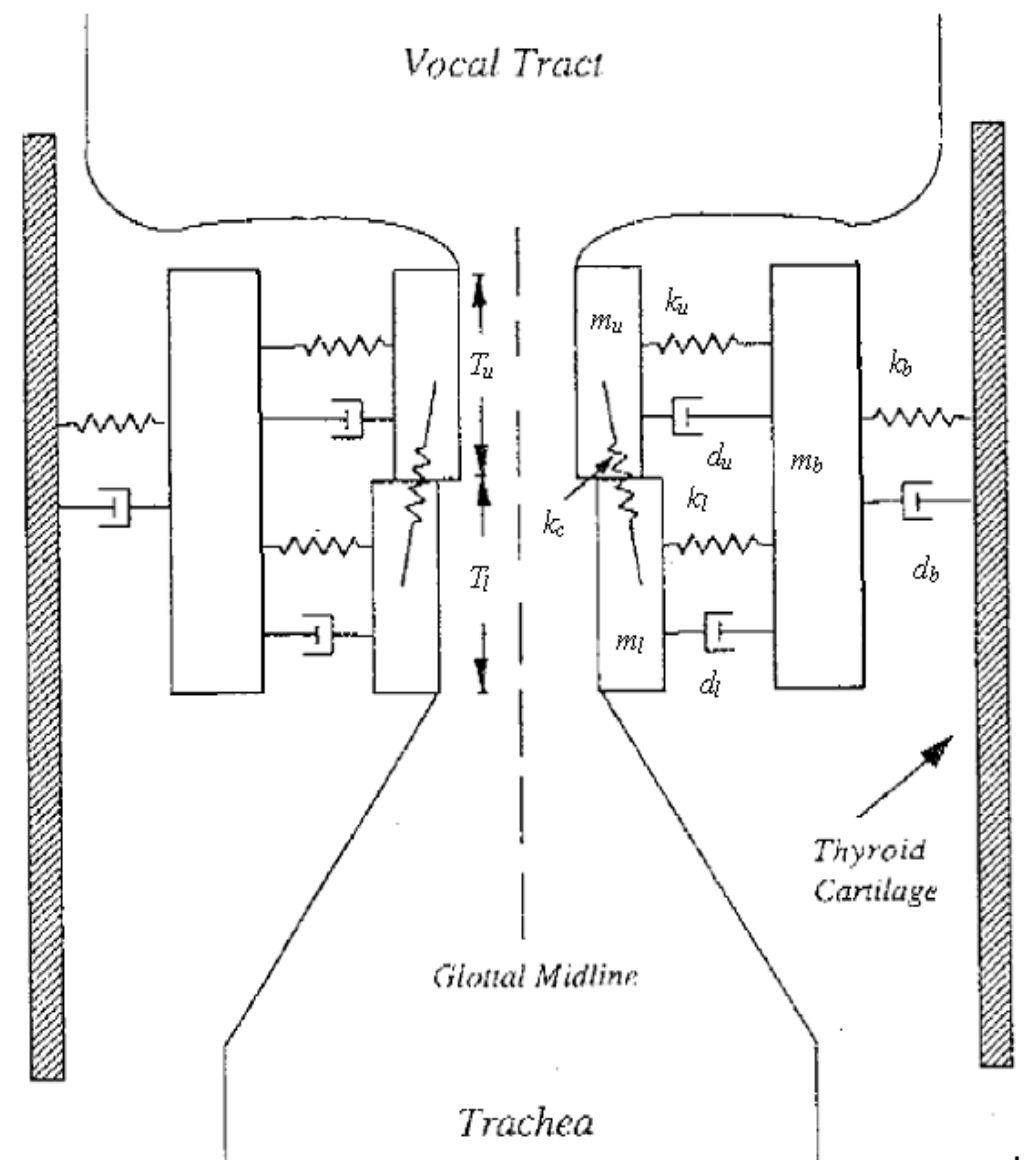


Figure 1.10 - Lumped-element representation of the body-cover structure of the vocal folds [18].

The equations that describe the dynamics of this system can be written as follows:

$$
\left\{\begin{array}{l}
F_{u}=m_{u} x_{u}^{\prime \prime}=F_{k u}+F_{d u}-F_{k c}+F_{e u}+F_{u C o l} \\
F_{l}=m_{l} x_{l}^{\prime \prime}=F_{k l}+F_{d l}+F_{k c}+F_{e l}+F_{l C o l} \\
F_{b}=m_{b} x_{b}^{\prime \prime}=F_{k b}+F_{d b}-\left[F_{k u}+F_{d l}+F_{k l}+F_{d l}\right]
\end{array}\right.
$$

where the following forces are identified as

$F_{d u}, F_{d l}$ and $F_{d b} \quad$ forces due to damping

$F_{k u}, F_{k l}$ and $F_{k b} \quad$ lateral spring forces

$F_{k c}$ spring force due to the coupling of $m_{u}$ and $m_{l}$

$F_{u C o l}$ and $F_{l C o l}$ forces generated only during collision with the opposite vocal fold $F_{e u}$ and $F_{e l}$ external forces generated by the glottal flow $F_{u}, F_{l}$ and $F_{b}$ forces of the accelerating masses

The equations of motion (1.3) describe the collision force between each vocal fold. Because the whole cover portion is replaced by two brick-shaped masses and concentrates into a few point masses, the collision force calculated in this model is limited to the transient collision period and causes an instantaneous stop, which is quite different from the real situation. Although nonlinear springs and collision dampers [15] are used in the ST95 model to increase the energy losses during the collision, it is hard to find the correlation between the physiologically realistic model and ST95's components. Another problem is the result of the impact stress, which is a very important parameter for vocal fold damage. Because the contact masses are brickshaped, the contact area of ST95 remains constant during collision, which is highly unnatural and thus the impact stress calculated or measured in ST95 is meaningless.

However, because of the simplification of the two-mass model, most study of speech synthesis has been based on the two-mass system. The challenge of this model is how to define the components' parameters. From 1994 [19, 20], scientists started to use FEA model to determine realistic values for the two-mass model's parameters. Furthermore, 
Jiang and Qiu [21] used FEA model to obtain more data including both healthy vocal folds and pathological vocal folds. Their results show that the two-mass system can simulate the vibration behaviour of normal and pathological vocal folds in a more realistic way. Nevertheless, the usage of the lumped mass system is limited for two reasons: First, it is simple brick-shaped geometries and spring-damper components (as shown in Figure 1.9, Figure 1.10). Second there is no direct correlation between the spring stiffness, damper viscosity and the tissue properties. A better model is required to predict the geometry and the viscoelastic properties of the vocal folds adequately and to investigate the voice disorders or special vocal qualities.

Continuum models of the vocal folds have also been considered. The advantage of continuum models is their ability to directly relate the geometric, viscoelastic, and aerodynamic characteristics of the vocal folds. In 1996, Berry and Titze [22] developed a continuum model of vocal fold tissues which was built to calculate eigenmodes and eigenfrequencies. The folds were assumed to be the simplest shape of a rectangular parallelepiped. In this model, the basic equations of motion, the boundary conditions, and material properties were defined. The potential energy and the Ritz method [22] are introduced to solve the continuum mechanical problem.

In traditional continuum mechanics, FEA is a widely used method to solve the problems. The advantages of FEA are the ability to handle complex boundaries and driving forces. The potential energy is based on the principle of virtual work, which is a fundamental statement of mechanics. Ritz method was elaborated by Courant [23, 24] in 1943, which is an approximate solution to simple geometric shapes while FEA utilises such concepts and extends that to complex structures by discretizing the geometry into simple finite elements which can be solved numerically with given boundary conditions. Alipour [25] developed a FEA model of the vocal fold from the basic laws of continuum mechanics to obtain the oscillatory characteristics of the vocal folds. This model is able to describe the irregular geometry, layered structure and anisotropic viscoelastic material properties as a completion of Berry and Titze's model [22]. Alipour assumed all vocal fold tissues are transverse isotropic (such as fibre) and the longitudinal movements of are restricted by defining the longitudinal Poisson's ratio as zero. Therefore, only the displacement in transversal planes was calculated in this model. The simplification reduced all nodes freedom from three directions to two directions. Additionally, the collision between the vocal folds is obtained by bounding the tissue displacement: when the surface nodes 
touch each other, they lose one degree of freedom in order to avoid the vocal folds penetrating into each other.

In 2002, Gunter set up a FEA model of the vocal folds collision with high spatial and temporal resolution [26]. This model has the spatial resolution that could calculate the submillimeter scale of vocal-fold displacement and represent the small size of nodules, injure and surgery repair. The temporal resolution could capture the submillisecond time scale of the vocal folds' collision and show the stage of the collision process explicitly. Gunter assumed that the model was an entire mass made of isotropic, linear elastic material instead of the layered structure and viscoelastic material. In her research, the initial conditions are that the vocal folds were assumed to be in static excited by a certain account of airflow and possessing no self-oscillation. The impact stress was calculated between the rigid midline surface and the vocal fold. In the real case, the impact stress is reduced and the contact time increased because of the effect of the material's viscosity.

The first physical model of the larynx was made to study intraglottal pressure profiles and asymmetric motions [27]. That model is 7.5 times larger than the normal vocal folds and made of Plexiglas which is a stiff material. It is not suited to studying the dynamic characteristics. However, a physical model which could represent the human vocal folds closely is given available by Thomson [28]. It is made of a three-component liquid polymer which is a mixture of a 2-part polyurethane addition cure polymer and Everflew $^{\mathrm{TM}}$. A high-speed digital camera was employed to obtain sequential images of the model motion. Because of the geometrical, mechanical and dynamic similarities to the human vocal folds, the results of the model are similar to the data from anatomical samples. Further study of the model [29] shows that the model vocal folds behaviour is close to actual physiological data. As shown in Figure 1.11, this model still can't represent the layered structure and the lateral shape should be a curve surface instead of a flat plane. 

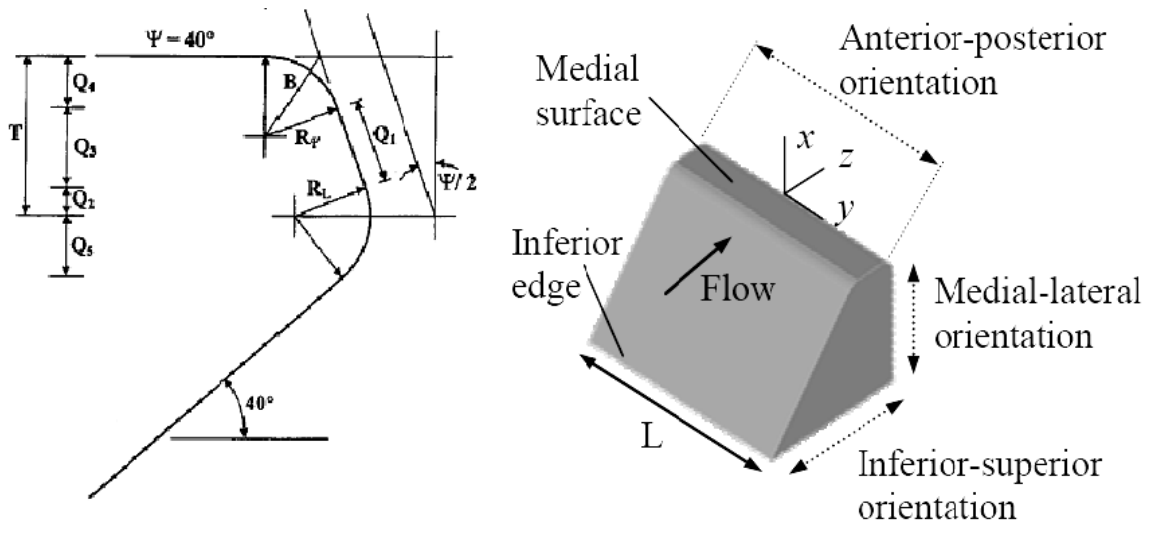

Figure 1.11 - Model vocal folds [29]

Although the above methods are good and physically acceptable, nevertheless they lack generality and accuracy. The mathematical models are based on either two-dimensional geometry or too simplified materials. The physical model is good to represent the physiological situation closely. But it is too complex to simulate pathological vocal folds. In this thesis, a more general and accurate model is proposed and compared with other available model. The model will be three-dimensional and made of nonlinear materials.

\subsection{Objective}

The main objective of this research is to calculate the frequency characteristics of the vocal folds and dynamic response during phonation. To achieve this goal, the following work is undertaken:

1) Build a complete FEA model to simulate the vocal folds and determine the geometry and material parameters in the model.

2) Validate the FEA model for simulation.

3) Compare the frequency results from the mathematical model and the experimental results of the silicone model and study the frequency characteristics of the vocal folds. 
4) Compare the dynamic results from the mathematical model and the experimental results and study the dynamic response of the vocal folds. 


\section{Chapter 2 FINITE ELEMENT MODEL}

\subsection{Introduction}

Nowadays numerical modelling plays an important role in research and development. A detailed three-dimensional layered theoretical model which incorporates the geometrical structure is described in this chapter. To represent the submillimeter and submillisecond scale of the collision process between two vocal folds during phonation, the finite element method is applied. The geometry of the model is based on the anatomical structure of human's vocal folds and described by series of simplified equations. According to the cover-body theory [12], the theoretical model is divided into two layers. All the materials assigned on the model are biomechanical materials. As discussed in section 1.2, the mucosa layer is assumed to be isotropic viscoelastic material; however, the muscle part is assumed transverse isotropic elastic. The finite element method is used to calculate the dynamic motion during phonation process.

\subsection{Geometrical Model}

The geometry of the proposed model is one of the most important part of the modelling process. Because this research focuses on the collision between two vocal folds, the other parts of the oral organ such as the cartilages of larynx and so on are ignored in the model. As most parts of the human organs, the geometry of the vocal folds in the human body is standard nonlinear. Simplified equations and cover-body theory are applied in building the geometry.

\subsubsection{Mathematical Description of the Vocal Fold}

In the literature, the structure of the vocal fold is mostly based on Titze's theory [30], which uses the nominal parameters based on the average male's vocal folds measured in vitro to describe the structure. The model is defined in Cartesian coordinates as shown 
in Figure 2.1. The $x$ dimensions indicate lateral distance from the glottal midline, $5 \mathrm{~mm}$ wide anteriorly, and $10 \mathrm{~mm}$ wide posteriorly. The $y$ dimensions define the distance from the posterior to anterior, which is $14 \mathrm{~mm}$ long.

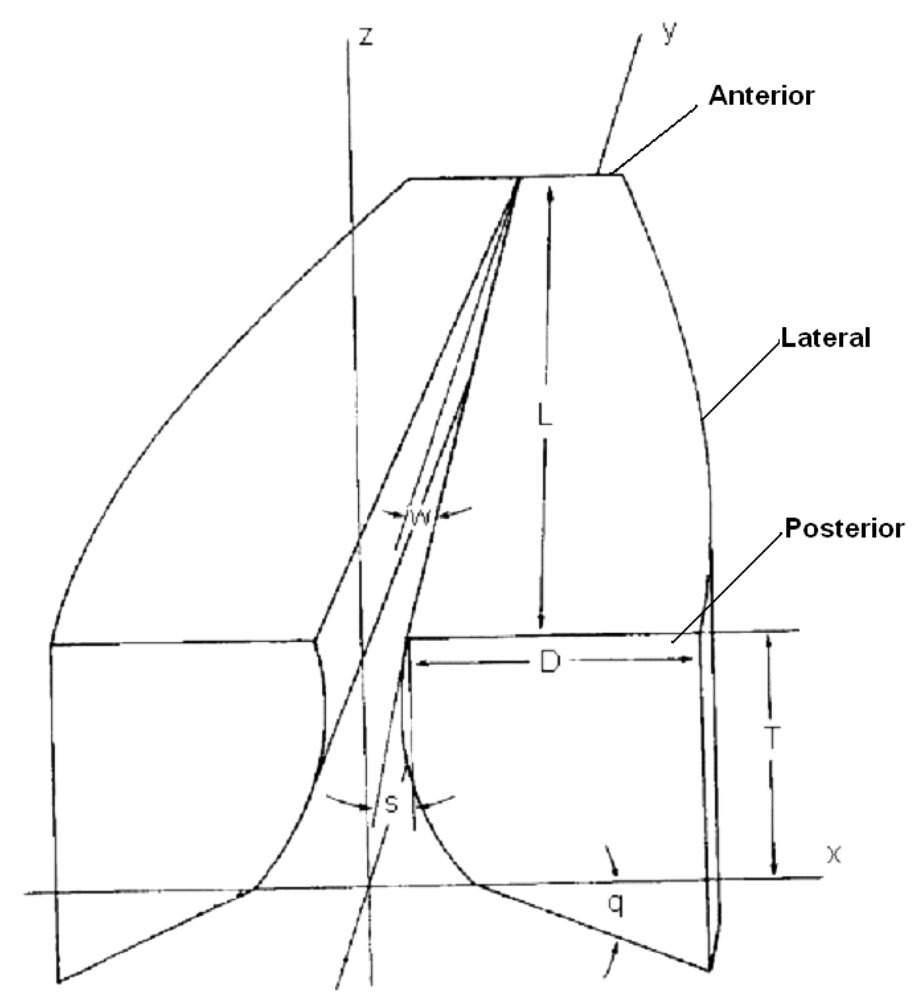

Figure 2.1 - Vocal folds structure based on Titze's theory [27, 29]

The parameters from Figure 2.1 are defined as follows:

$\mathrm{L}=$ the length of vocal folds as measured from the vocal processes to the anterior commissure; nominal value $14 \mathrm{~mm}$.

$\mathrm{T}=$ the thickness of the vocal folds, including the top mucosal layer, the ligament, and a portion of the conus elastics region; nominal value $5 \mathrm{~mm}$.

$\mathrm{D}=$ the depth of the vocal folds, decreasing from a maximum at the vocal processes $(10$ $\mathrm{mm}$ ) to a minimum (5 $\mathrm{mm}$ ) according to the following factor [30]

$$
f=1-0.5 y^{2} / L^{2}
$$

$y=$ the displacement from the anterior surface to the node 
$w=$ the angular deviation of the superior-medial edge with respect to the vertical midplane of the glottis. This parameter adjusts the position of the vocal process, and therewith the superior vocal-fold abduction; nominal value $0.0 \mathrm{rad}$.

$\mathrm{s}=\mathrm{a}$ shaping factor, which controls the remainder of the medial surface contour with respect to the vertical mid-plane of the glottis. The nominal value of parameter $\mathrm{s}$ is $0.05 \mathrm{rad}$. The contour is quadratic and provides additional abduction below the superior surface, as shown in Figure 2.1.

Mathematically the two dimensional glottal width $\mathrm{g}$ is given by [30]

$$
g(y, z)=2 w(L-y)+2\left[-s(T-z)+\left(T-z^{2}\right)\right] f
$$

To simplify Equation 2.2, the following equation is used [26].

$$
g(0.5 m m \leq h \leq 5 m m)=0.45+0.19(h-5)+0.02(h-5)^{2}
$$

where $h(\mathrm{~mm})$ is the vertical distance below the superior surface. Equation 2.2 uses three variables while Equation 2.3 contains just one variable which can be determined from an experimental set-up. The effect of this simplification which could be determined experimentally will be discussed later.

The minimum glottal width of $0 \mathrm{~mm}$ that occurs at the inferior end of the fillet (equal to $0.5 \mathrm{~mm}$ ) indicates that the undeformed vocal fold is tangent to the glottal midline. To create a smooth contour in the coronal plane, this geometry is modified by applying a fillet with a radius of $0.5 \mathrm{~mm}$ to the superior medial curve.

\subsubsection{Building the Model Using Solidworks}

Using the parameters listed above, it is possible to build the geometry part of the model in Solidworks as required. However, Solidworks doesn't support geometry equations as an input. To solve this problem, the vocal fold's upper part $(z \geq 0)$ is divided by six parallel planes from $z=0$ to $z=5 \mathrm{~mm}$. All the points on the planes which are calculated from Equation 2.1, Equation 2.2 and Equation 2.3 by Matlab are shown in Table AI.1.

Figure 2.2 and Figure 2.3 show drawings based on the data from Table AI.2 and Table AI.3. For each drawing the spline function was used to connect the points to form a smooth curve and a straight line was used to connect the curve at the end points. 


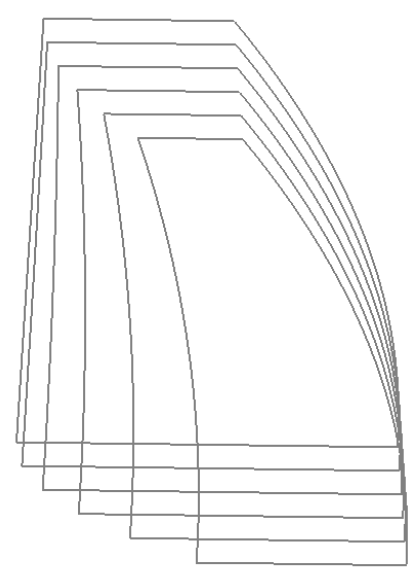

Figure 2.2 - Drawing based on Equation 2.2

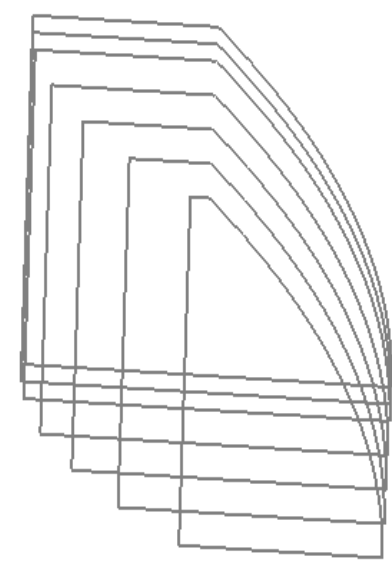

Figure 2.3 - Drawing based on Equation 2.3

The upper part $(z>0 \mathrm{~mm})$ of the model is built by using the Loft function to connect the sketches together. The stratum part is formed by extruding the bottom sketch (the sketch at plane $z=0 \mathrm{~mm}$ ) and drafting the extension part by the angle $q=0.7$ radians. A fillet with a radius of $0.5 \mathrm{~mm}$ is applied to the superior medial curve in order to create a smooth contour in the coronal plane. Figure 2.4 and Figure 2.5 show the final solid bodies for model 1 and model 2 respectively.

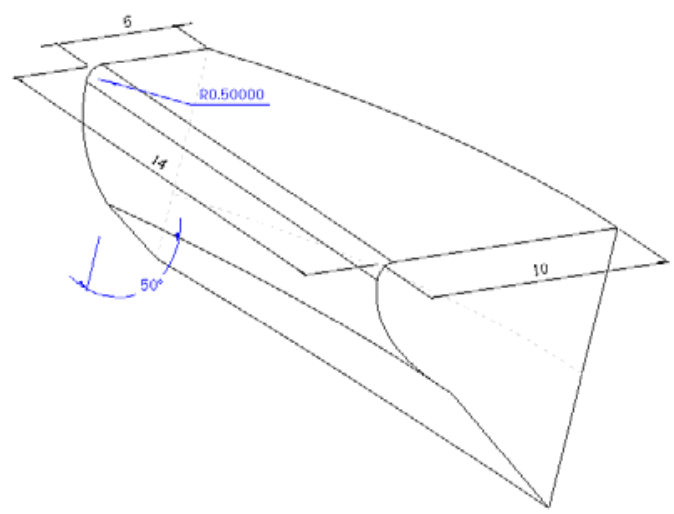

Figure 2.4 - Model 1 based on Equation 2.2 


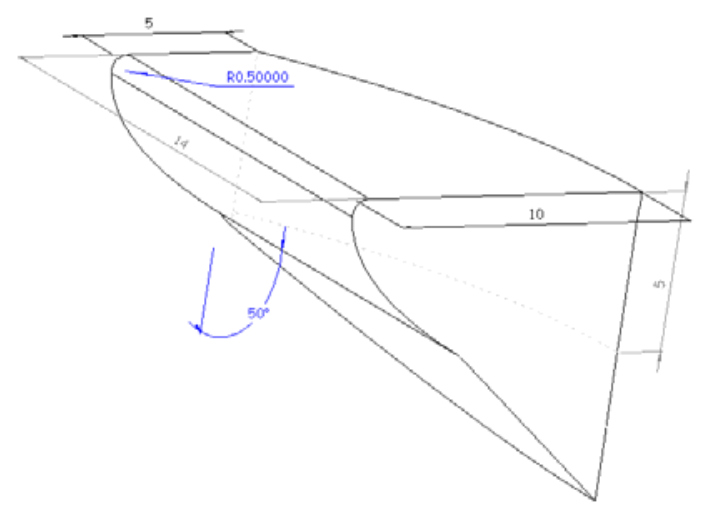

Figure 2.5 - Model 2 based on Equation 2.3

\subsubsection{Testing of Model Geometry in ABAQUS}

The simplification of geometry should be acceptable without sacrifice in accuracy. Therefore, Model 1 and model 2 have been tested by using ABAQUS.

In the test, the material properties are isotropic and linear, with a Young's modulus $E$ of $36.1 \mathrm{kPa}$, a Poisson's ratio $v$ of 0.3 and a density of $1.1 \mathrm{~g} / \mathrm{cm}^{3}$. These parameters are based on experimental measurements on human vocal ligament samples by Min [31], and are valid for strains of less than $15 \%$. In the two models, the anterior, posterior, and lateral surfaces are fixed to represent their attachment to the laryngeal cartilages.

Table 2-1 - Natural frequencies of model 1 and model 2

\begin{tabular}{|c|c|c|}
\hline Natural Frequency & Model 1 & Model 2 \\
\hline First & $135.86 \mathrm{~Hz}$ & $130.33 \mathrm{~Hz}$ \\
\hline Second & $218.21 \mathrm{~Hz}$ & $211.61 \mathrm{~Hz}$ \\
\hline Third & $231.61 \mathrm{~Hz}$ & $232.29 \mathrm{~Hz}$ \\
\hline Forth & $249.58 \mathrm{~Hz}$ & $247.62 \mathrm{~Hz}$ \\
\hline Fifth & $262.36 \mathrm{~Hz}$ & $260.60 \mathrm{~Hz}$ \\
\hline
\end{tabular}

The results show that, there is no significant difference between the two models. The natural frequency of an adult male vocal fold is about $127 \mathrm{~Hz}$, which was measured in vivo and was consistent with resonance frequencies as measured by Kaneko [32]. Model 2's first frequency is quite near the experimental data. Therefore, the FEA model and experimental sample will be based on model 2, Equation 2.3. 


\subsubsection{Cover and Body Theory}

The vocal fold's structure has been shown to be multilayered (refer to section 1.2.2). Following the cover-body theory discussed in Chapter 1, the whole vocal fold is divided into two parts, the cover part and the body part. The thickness of the cover is $1 \mathrm{~mm}$ at the posterior portion and tapers linearly to half of this value at the anteriority. The thickness of the cover part is modelled by using the following equation.

$$
d=(28-y) * 1.0 \mathrm{~mm} / 28
$$

$d=$ the thickness of the cover

The eventual Solidworks cover part and the body part are shown in Figure 2.6 and Figure 2.7 .

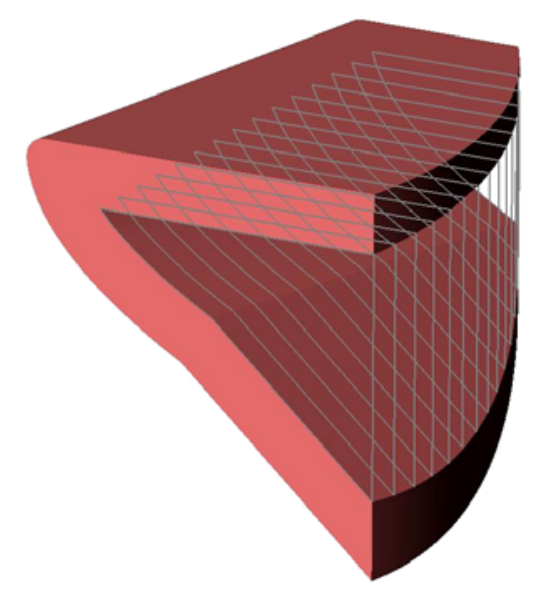

Figure 2.6 - Cover part of the model

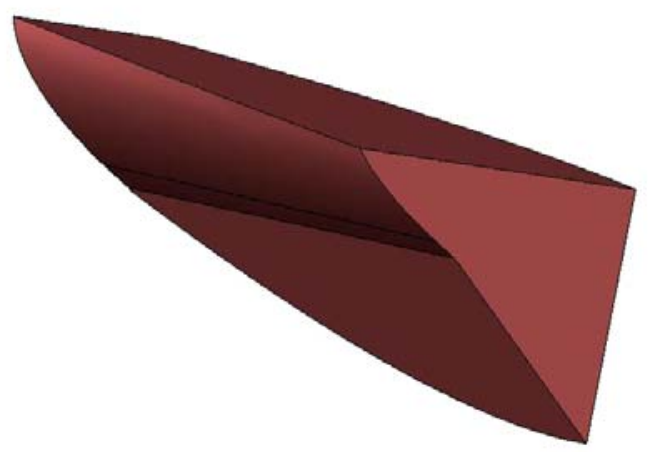

Figure 2.7 - Body part of the model

The ideal model is capable of representing all possible healthy or pathologic situations of the vocal folds. However, using in the current techniques, it is hard to achieve that 
purpose. In this study, the healthy situation is considered. Commonly, it is observed that most healthy human vocal folds are symmetric. As shown in Figure 2.8, the model is layered and symmetric.

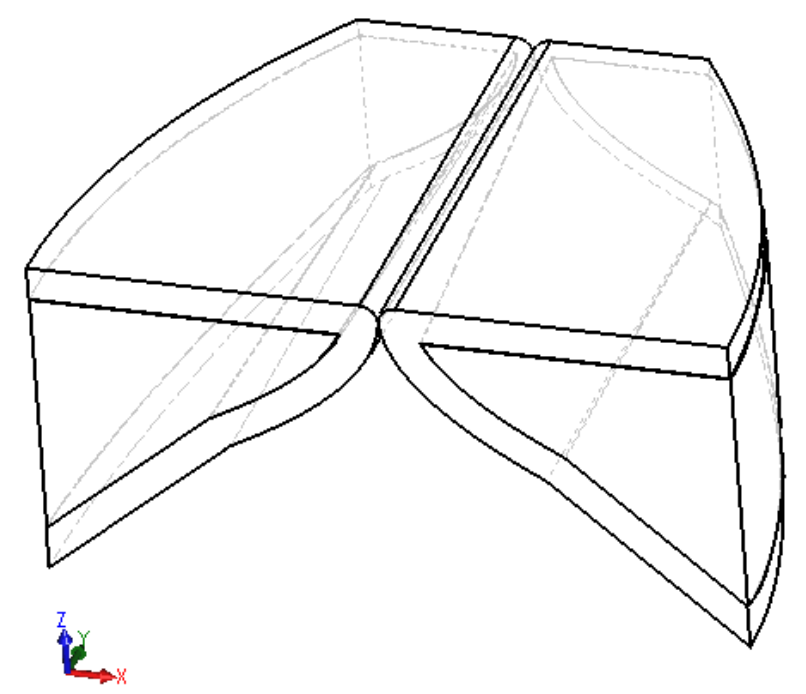

Figure 2.8 - A pair of layered vocal folds model

\subsection{Materials Definition in the Model}

As illustrated in Chapter 1, the cover part of the vocal fold is made of loosely organized microfibrils small components and softer, fluid-like tissue. A material which exhibits both "elastic" and "viscous" properties is called a viscoelastic material. In the current literature, the vocal folds' cover part is normally considered to be a viscoelastic material. Furthermore, the viscoelastic and isotropic material is usually assumed to be the simplest viscoelastic material: linear, isotropic viscoelastic material.

Most research shows that the dynamic viscoelastic behaviour depends on temperature [33]. For biological tissues, an analogous range of temperature is located at $5-37{ }^{\circ} \mathrm{C}$, in which there is no structural change of tissues [34]. The vocal folds' temperature depends on the exhaled air flow temperature and the body temperature. A typical breathing cycle temperature distribution is shown in Figure 2.9 [35]. 


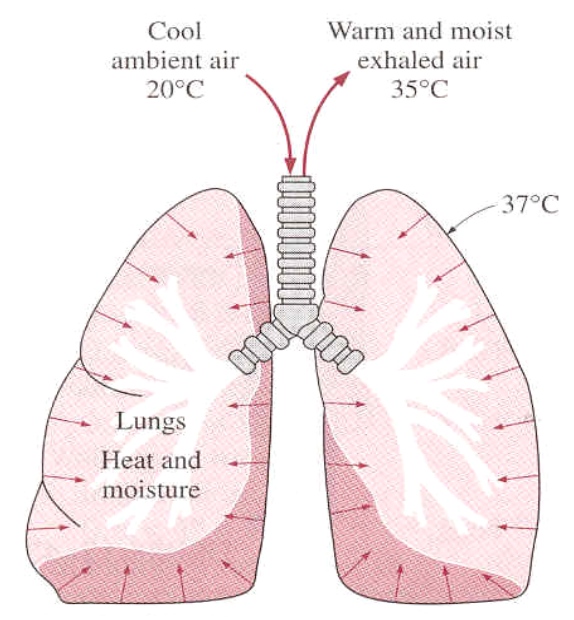

Figure 2.9 - Temperature of the breathing system [35]

Exhaled air which vibrates the vocal folds during phonation is almost fully saturated with moisture at the body temperature of $35^{\circ} \mathrm{C}$ regardless of the breathing rate. In a living human, the body temperature is about $37^{\circ} \mathrm{C}$ and the vocal fold's temperature should be between the body temperature and the exhaled air temperature, which is about $35-37^{\circ} \mathrm{C}$. Therefore, the temperature effect on the cover material is ignored.

The body part of the vocal fold consists of the deep layer and the muscle fibres. As discussed in Chapter 1, these fibres are nearly inextensible and run parallel to the anterior-posterior direction. As for most fibre-like materials, the fibre body tissue is generally stiffer in the direction of fibres. Furthermore, it is observed that the vocal folds vibration occurs mainly at the surface of the thin layer of the non-muscular tissue [18]. This means that the viscosity of the vocal folds' body part plays a small role in the vocal folds' vibration process. Therefore, the body part is assumed to be pure elastic transverse isotropic material [36]. On the basis of this assumption, the defined mechanical properties are independent of the orientation of deformation (isotropy) in one plane (x-z plane in this model) transverse to the essentially parallel tissue fibres, but different in the longitudinal direction (y direction in this model) of the fibres. The direction of the model is shown in Figure 2.8.

The following two sections will describe the mathematical model of different materials in finite element analysis. In these two sections both elastic and viscoelastic parameters of the body part and the cover part are defined. 


\subsubsection{Elastic Material (body part)}

The body part mainly consists of the thyroarytenoid muscle. In a small strain situation, the human muscles are assumed to be linear and transverse isotropic materials. The conversion of stress to displacement follows Hooke's law as

$$
\sigma=[S] \varepsilon
$$

where $\sigma$ is stress, $\varepsilon$ is strain tensor, and $[S]$ is a stiffness matrix. In general, $[S]$ is a $6 \times 6$ symmetric matrix with 21 independent constants. For the case of transverse isotropic material about the $y$ axis, the inverse of $[S]$ could be expressed in terms of five independent mechanical constants [37]. The stress tensor is expressed as,

$$
\sigma=\left(\begin{array}{llllll}
\sigma_{x} & \sigma_{y} & \sigma_{z} & \tau_{x y} & \tau_{y z} & \tau_{z x}
\end{array}\right)^{T}
$$

the strain as,

$$
\varepsilon=\left\{\begin{array}{c}
\partial u / \partial x \\
\partial v / \partial y \\
\partial w / \partial z \\
\partial y / \partial x+\partial x / \partial y \\
\partial z / \partial y+\partial y / \partial z \\
\partial x / \partial z+\partial z / \partial x
\end{array}\right\}
$$

and the material properties as,

$$
[S]=\left(\begin{array}{cccccc}
E_{1111} & E_{1122} & E_{1133} & 0 & 0 & 0 \\
& E_{2222} & E_{2233} & 0 & 0 & 0 \\
& & E_{3333} & 0 & 0 & 0 \\
s y m m & & & E_{1212} & 0 & 0 \\
& & & & E_{2323} & 0 \\
& & & & & E_{3131}
\end{array}\right)
$$

Due to the isotropic and linear assumptions, the parameters in $[S]$ can take the form 


$$
\left\{\begin{array}{l}
E_{1111}=E_{3333} \\
E_{1122}=E_{2233} \\
E_{1212}=E_{2323} \\
E_{3131}=\frac{1}{2}\left(E_{3333}-E_{1133}\right)
\end{array}\right.
$$

Equation (2.10) is inversed to obtain the displacements expressed by stresses,

$$
\begin{gathered}
\varepsilon=[S]^{-1} \sigma \\
\left(\begin{array}{c}
\partial u / \partial x \\
\partial v / \partial y \\
\partial w / \partial z \\
\partial y / \partial x+\partial x / \partial y \\
\partial z / \partial y+\partial y / \partial z \\
\partial x / \partial z+\partial z / \partial x
\end{array}\right)=\left(\begin{array}{cccccc}
1 / E & -v^{\prime} / E^{\prime} & -v / E & 0 & 0 & 0 \\
-v^{\prime} / E^{\prime} & 1 / E^{\prime} & -v^{\prime} / E^{\prime} & 0 & 0 & 0 \\
-v / E & -v^{\prime} / E^{\prime} & 1 / E & 0 & 0 & 0 \\
0 & 0 & 0 & 1 / \mu^{\prime} & 0 & 0 \\
0 & 0 & 0 & 0 & 1 / \mu^{\prime} & 0 \\
0 & 0 & 0 & 0 & 0 & 1 / \mu
\end{array}\right)\left(\begin{array}{c}
\sigma_{x} \\
\sigma_{y} \\
\sigma_{z} \\
\tau_{x y} \\
\tau_{y z} \\
\tau_{z x}
\end{array}\right)
\end{gathered}
$$

where $E$ is the Young's modulus in the transverse plane, $E^{\prime}$ is the Young's modulus along the y axis, $\mu^{\prime}$ is the shear modulus along the y axis, $v$ is the Poisson's ratio in the transverse plane, and $v^{\prime}$ is the Poisson's ratio along the y axis.

Another constant $\mu$ (the shear modulus in the transverse plane), can be expressed by

$$
\mu=\frac{E}{2(1+v)}
$$

In a continuum model, the solutions are usually calculated by differential or partial differential equations under certain boundary conditions. This requires regular boundary geometry. However, it is difficult to impose boundary conditions on the function if the boundary geometry is non-linear and irregular. In finite element method, the problem is solved by assigning the boundary conditions along the boundary nodes.

In a three-dimensional model, a tetrahedral element is one of the simplest and practical ways to mesh the complicated geometry. The state of displacement of a point is defined by three displacement components, $u, v$, and $w$, in the directions of the three coordinates $x, y$, and $z$ respectively. Thus 


$$
U=\left\{\begin{array}{l}
u \\
v \\
w
\end{array}\right\}
$$

Using the finite element concept, the displacements within an element have to be uniquely defined by these twelve values $\left(\alpha_{1} \sim \alpha_{12}\right)$. The simplest representation is clearly given by three linear polynomials

$$
\left\{\begin{array}{c}
u=\alpha_{1}+\alpha_{2} x+\alpha_{3} y+\alpha_{4} z \\
v=\alpha_{5}+\alpha_{6} x+\alpha_{7} y+\alpha_{8} z \\
w=\alpha_{9}+\alpha_{10} x+\alpha_{11} y+\alpha_{12} z
\end{array}\right.
$$

$i, j, m, p$ are four nodes on the tetrahedral element as shown in Figure 2.10.

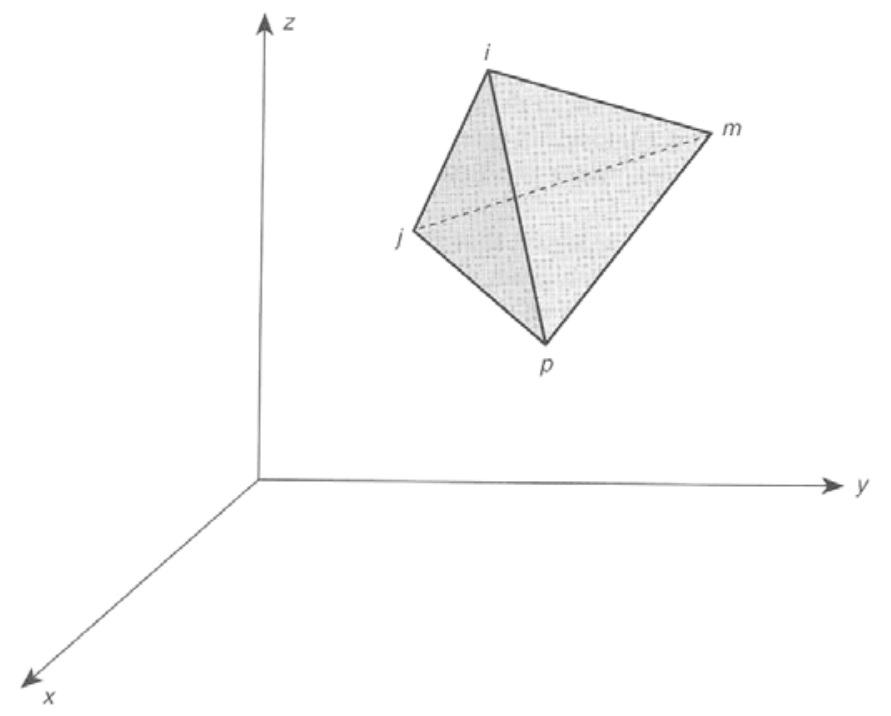

Figure 2.10 - A tetrahedral volume

Equating the values of the displacements of the nodes in $u$ direction, we have four equations of the type

$$
\left\{\begin{array}{c}
u_{i}=\alpha_{1}+\alpha_{2} x_{i}+\alpha_{3} y_{i}+\alpha_{4} z_{i} \\
u_{j}=\alpha_{1}+\alpha_{2} x_{j}+\alpha_{3} y_{j}+\alpha_{4} z_{j} \\
u_{m}=\alpha_{1}+\alpha_{2} x_{m}+\alpha_{3} y_{m}+\alpha_{4} z_{m} \\
u_{p}=\alpha_{1}+\alpha_{2} x_{p}+\alpha_{3} y_{p}+\alpha_{4} z_{p}
\end{array}\right.
$$


The displacement field can be written for each element as

$$
\left\{\begin{array}{c}
u=N_{i} u_{i}+N_{j} u_{j}+N_{m} u_{m}+N_{p} u_{p} \\
v=N_{i} v_{i}+N_{j} v_{j}+N_{m} v_{m}+N_{p} v_{p} \\
w=N_{i} w_{i}+N_{j} w_{j}+N_{m} w_{m}+N_{p} w_{p}
\end{array}\right.
$$

The interpolation function (shape function) $N_{i}, N_{j}, N_{m}, N_{p}$ consists of four linear polynomials, defining the shape of the displacement field for each tetrahedral element. In this model the linear shape functions are assumed to be

$$
\left\{\begin{array}{cc}
N_{i}=\frac{\left(a_{i}+b_{i} x+c_{i} y+d_{i} z\right)}{6 V} & (i, m) \\
N_{j}=-\frac{\left(a_{j}+b_{j} x+c_{j} y+d_{j} z\right)}{6 V} & (j, p)
\end{array}\right.
$$

where $V$ is the volume of the element which can be calculated by

$$
V=\frac{1}{6} \operatorname{det}\left|\begin{array}{cccc}
1 & x_{i} & y_{i} & z_{i} \\
1 & x_{j} & y_{j} & z_{j} \\
1 & x_{m} & y_{m} & z_{m} \\
1 & x_{p} & y_{p} & z_{p}
\end{array}\right|
$$

and $a_{i}, b_{i}, c_{i}, d_{i}$ are coefficients calculated from the nodal coordinates.

$$
\begin{array}{ll}
a_{i}=\operatorname{det}\left|\begin{array}{lll}
x_{j} & y_{j} & z_{j} \\
x_{m} & y_{m} & z_{m} \\
x_{p} & y_{p} & z_{p}
\end{array}\right| & b_{i}=-\operatorname{det}\left|\begin{array}{lll}
1 & y_{j} & z_{j} \\
1 & y_{m} & z_{m} \\
1 & y_{p} & z_{p}
\end{array}\right| \\
c_{i}=-\operatorname{det}\left|\begin{array}{lll}
x_{j} & 1 & z_{j} \\
x_{m} & 1 & z_{m} \\
x_{p} & 1 & z_{p}
\end{array}\right| & d_{i}=-\operatorname{det}\left|\begin{array}{lll}
x_{j} & y_{j} & 1 \\
x_{m} & y_{m} & 1 \\
x_{p} & y_{p} & 1
\end{array}\right|
\end{array}
$$

The shape function would be solved by substituting Equation (2.15) into Equation (2.14). The stiffness and mass matrices will be defined in ABAQUS. The nodal force vector acting on each element is the aerodynamic pressure force, which can be calculated from Equation (2.5) by using the corresponding nodes. 


\subsubsection{Viscoelastic Material (cover part)}

Viscoelastic phenomena are characterized by the fact that the rate at which inelastic strain depends not only on the current state of stress and strain, but, in general, also on the full history of their development.

The constitutive equation for linear viscoelasticity can be given in the form of a differential equation. The constitutive equation is written as a linear elastic part with an added series of partial strains $q$.

$$
\sigma(t)=D_{0} \varepsilon(t)+\sum_{m=1}^{M} D_{m} q^{(m)}(t)
$$

where for a linear model the partial stresses are solutions of the first-order differential equations

$$
\dot{q}^{(m)}+T_{m} q^{(m)}=\dot{\varepsilon}
$$

with $T_{m}$ a constant matrix of reciprocal relaxation times and $D_{0}, D_{m}$ constant moduli matrices.

Equation (2.19) is suitable for both isotropic and anisotropic linear viscoelastic materials. To describe more detail about isotropic models, the stress is split into two terms, as follows:

$$
\sigma=s+m p
$$

where $s$ is the stress deviator, $p$ is the mean stress and $m$ is $\left[\begin{array}{llllll}1 & 1 & 1 & 0 & 0 & 0\end{array}\right]^{T}$. The deviatoric part is stated as differential equation models, in which the constitutive equation may be written as

$$
s=2 G\left(\mu_{0} e+\sum_{m=0}^{M} \mu_{m} q^{(m)}\right)
$$

where $e$ is the stress deviator and $\mu_{m}$ are dimensionless parameters satisfying 


$$
\sum_{m=1}^{M} \mu_{m}=1
$$

The integral equation form for the deviatoric stresses is expressed in terms of a relaxation modulus function which is defined by an idealized experiment, in which, at time $(t=0)$, a specimen is subjected to suddenly applied and constant strain, $e_{0}$, and the stress response, $s(t)$ is measured. For linear material, the relation obtained is as follow,

$$
s(t)=2 G(t) e_{0}
$$

where $G(t)$ is the shear relaxation modulus function. $G(t)$ could be described by the form of generalization to the Maxwell model, which is in a Prony series form

$$
G(t)=G\left[\mu_{0}+\sum_{m=1}^{M} \mu_{m} \exp \left(-t / \lambda_{m}\right)\right]
$$

where $\lambda_{m}$ are relaxation times. However, the time domain data is hard to obtain from experiment as the vocal fold mucosa is too small as a test material, so frequency dependent test data was expected to replace them. The conversion of the test data to Prony series will be described in ABAQUS model building.

\subsection{Equilibrium Equations of the Model}

The following sections state the equilibrium equations of motion, contact definition, and boundary conditions to solve the finite element model.

\subsubsection{Equations of Motion}

For infinitesimal displacements in an elastic continuum object, Newton's second law of motion is written as 


$$
\left\{\begin{array}{l}
\frac{\partial \sigma_{x}}{\partial x}+\frac{\partial \tau_{x y}}{\partial y}+\frac{\partial \tau_{z x}}{\partial z}+F_{x}=\rho \frac{\partial^{2} u}{\partial t^{2}} \\
\frac{\partial \tau_{x y}}{\partial x}+\frac{\partial \sigma_{y}}{\partial y}+\frac{\partial \tau_{y z}}{\partial z}+F_{y}=\rho \frac{\partial^{2} v}{\partial t^{2}} \\
\frac{\partial \tau_{z x}}{\partial x}+\frac{\partial \tau_{y z}}{\partial y}+\frac{\partial \sigma_{z}}{\partial z}+F_{z}=\rho \frac{\partial^{2} u}{\partial t^{2}}
\end{array}\right.
$$

where $u, v$ and $w$ are displacements in the $x, y$ and $z$ directions; $\rho$ is the density of the material; $\sigma_{i}$ are normal stresses; $\tau_{i j}$ are shear stresses $(i, j=x, y, z)$; and $F_{x}, F_{y}$ and $F_{z}$ are the body forces. Consequently, the general tissue displacement vector about the equilibrium position is written as

$$
\Psi(x, y, z, t)=u(x, y, z, t) i+v(x, y, z, t) j+w(x, y, z, t) k
$$

Equations (2.27) and (2.28) contain both stresses and displacements as variables. To solve the equations, either stresses or displacements should be chosen and converted to the others. The model of vocal-fold collision needs spatial resolution that is capable of representing an explicit vocal-fold collision. Therefore, it is more appropriate to use displacements as variable in this research. The relationship between stresses and displacements depends on the nature of the material.

However, using stress-displacement equations are too involved and a bit complicated to solve explicitly. Another numerically oriented approach is normally used. Virtual work principle is the numerically oriented approach of motion. Problems of elasticity are intimately related to the energy principles which define equilibrium conditions. The concept of energy principles is very important in the solution of elasticity problems. The potential energy of the deformed vibrating continuum is

$$
\pi=U-W_{p}
$$

where $U$ is the strain energy calculated from energy density as

$$
U=\iiint_{V} \frac{1}{2}\left(\sigma_{x} \varepsilon_{x}+\sigma_{y} \varepsilon_{y}+\sigma_{z} \varepsilon_{z}+\tau_{x y} \gamma_{x y}+\tau_{y z} \gamma_{y z}+\tau_{z x} \gamma_{z x}\right) d V
$$

and $W_{p}$ is the virtual work defined as, 


$$
W_{p}=\iiint_{V}\left(F_{x} u+F_{y} v+F_{z} w\right) d V+\iint_{A}\left(S_{x} u+S_{y} v+S_{z} w\right) d A
$$

In Equation (2.30), $F_{x}, F_{y}, F_{z}$ are components of the body force (inertia) and $S_{x}, S_{y}, S_{z}$ are components of the surface forces. The equations of motion are obtained by setting the variations of potential energy with respect to nodal displacement to zero. The virtual work principle is considered as a statement of mechanics more fundamental than the traditional equilibrium conditions of Newton's laws of motion. Therefore, in this model, the virtual work principle is used to solve the equilibrium of motions.

\subsubsection{Contact Problem}

The physical contact problem occurs when two or more bodies touch each other. In larynx, the vocal folds push and slide against each other, changing their shapes.

On the contact surfaces, the traction forces acting are called contact forces. The contact forces deform the objects. In this model, the simulation uses a contact algorithm (considering pure master-slave) to deal with the contact problem: all collided nodes (on the surface that interact with the other) are collected with the purpose of calculating the required forces to avoid body interpenetration. Such forces are calculated in terms of how deep one vocal fold can enter into each other. A typical iterative algorithm repeats two operations until it finds a stable solution as shown in Figure 2.11:

- Find the section where two objects penetrate.

- Apply forces to push back the penetrating section.
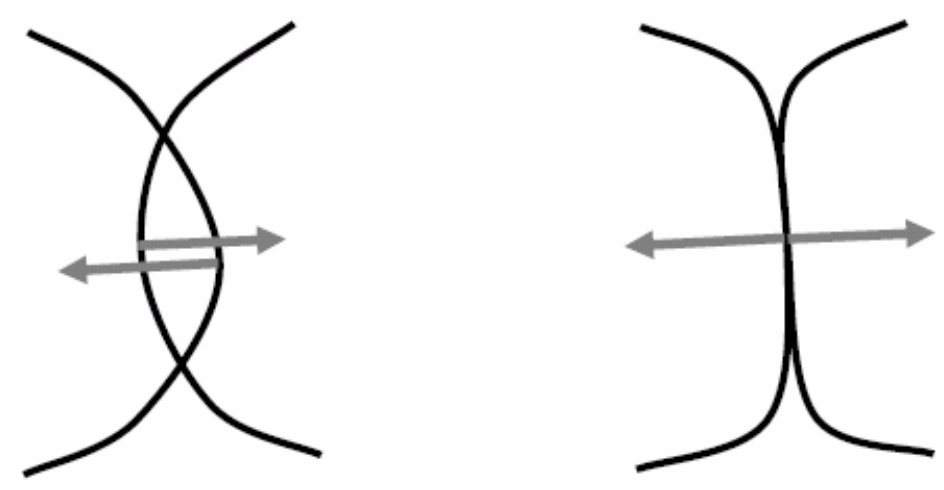

Figure 2.11 - Typical steps for solving the contact problem. 
The contact forces are the same as the push back forces in the final stable configuration. In conventional methods, the direction of a push back force, or the "normal", is chosen as the direction from a penetrating surface point to the closest projection.

Mathematically, all the collided nodes follow the restriction:

$$
\begin{aligned}
& G=(X-Y)^{T} * N \geq 0 \\
& L=F^{*} N \geq 0 \\
& G^{*} L=0
\end{aligned}
$$

$\mathrm{G}=$ the gap normal to the contact surface

$\mathrm{F}=$ the collision force

$\mathrm{N}=$ the unit normal vector of the contact surface

$\mathrm{L}=$ the contact force normal to the contact surface

It means that the gap $(G)$ between two nodes ( $X$ and $Y$ are the spatial position of two nodes on master and slave surfaces of the vocal folds) should be non-negative and that the collision force $F$ should always take the bodies apart. Equation (2.29) points out that both the inequalities are exclusive: when one condition is achieved, the other one is automatically equal to zero.

\subsubsection{Boundary Conditions}

As already mentioned, fixed boundaries are imposed at the lateral, anterior, and posterior surfaces, and free boundaries at the medial, superior, and inferior surfaces.

\subsection{ABAQUS Analysis}

This research focuses on figuring out the frequency characteristics and calculating the impact stress between two vocal folds during phonation. The impact force is a type of force in physics which describes the effect that time has on accelerating bodies.

In ABAQUS analysis, the following steps will be used to build a FEA model and do analysis.

- Build geometry parts 
- Define material properties

- Assemble model

- Define interaction

- Define steps

- Define load and boundary condition

- Mesh

- Results

\subsubsection{Build Geometrical Parts}

All of the geometrical parts are built by Solidworks and saved as igs file which can be imported by ABAQUS. See section 2.2.

\subsubsection{Define Material Properties}

Computer simulations of speech and voice demonstrate that knowledge of tissue mechanical properties is required to predict tissue forces and deformation. Standard human tissue is a viscoelastic material, which will increase computational costs greatly. It is necessary to simplify the material in order to reduce the cost.

\subsubsection{The Property of the Cover}

The density of human body is about 1 to $1.1 \mathrm{~g} / \mathrm{cm}^{3}$, because human body consists of 75\% water. Perlman's research (cited in Hunter, 2003, page1949 [38]) on the larynx gave an accurate value for the vocal folds (including the cover and body part) of $1.043 \mathrm{~g} / \mathrm{cm}^{3}$.

In ABAQUS, the elastic modulus is defined in "Linear elastic behaviour", with a Young's modulus $\left(E_{c}\right)$ of $41.9 \mathrm{kPa}$ (instantaneous modulus time scale for viscoelastic) and Poisson's ratio $(\rho)$ of 0.47 [39].

Frequency dependent test data are widely used in the study of viscoelastic properties. Chan and Titze [36] extensively investigated the vocal fold cover's properties. They obtained data of the elastic shear modulus $\left(G^{\prime}\right)$ and the viscous shear modulus $\left(G^{\prime \prime}\right)$ from 
a vocal fold cover tissue sample at frequencies of $0.01-15 \mathrm{~Hz} . G^{\prime}$ gives information about the elasticity or the energy stored in the material during deformation, whereas $G^{\prime \prime}$ describes the viscous character or the energy dissipated as heat. The dynamic viscosity $\eta$ is related to the loss modulus $G^{\prime \prime}$ by [36]:

$$
\eta=\frac{G^{\prime \prime}}{\omega}
$$

However, $G^{\prime}$ and $G^{\prime \prime}$ can't be used by ABAQUS directly. The Prony series is used for the definition of viscoelastic properties definition. It can also be calibrated by using frequency-dependent test data. The expressions for the shear moduli, obtained by converting the Prony series terms from the time domain to the frequency domain by making use of Fourier transforms, can be written as follows:

$$
\begin{gathered}
G_{s}(\omega)=G_{0}\left[1-\sum_{i=1}^{N} g_{i}^{p}\right]+G_{0} \sum_{i=1}^{N} \frac{g_{i}^{p} \tau_{i}^{2} \omega^{2}}{1+\tau_{i}^{2} \omega^{2}} \\
G_{s}(\omega)=G_{0} \sum_{i=1}^{N} \frac{g_{i}^{p} \tau_{i} \omega}{1+\tau_{i}^{2} \omega^{2}}
\end{gathered}
$$

Where $G_{s}(\omega)$ is the storage modulus, $G_{l}(\omega)$ is the loss modulus, $\omega$ is the angular frequency, and $\boldsymbol{N}$ is the number of terms in the Prony series. In comparison, the elastic shear modulus $\left(G^{\prime}\right)$ is the storage modulus; the viscous shear modulus $\left(G^{\prime \prime}\right)$ is the loss modulus.

In ABAQUS, the viscoelasticity defined by frequency needs the following data: $\omega \Re\left(g^{*}\right), \omega \xi\left(g^{*}\right), \omega \Re\left(k^{*}\right), \omega \xi\left(k^{*}\right), f$

1. Real part of $\omega^{*}$.

$$
\omega \Re\left(g^{*}\right)=G_{l} / G_{\infty}
$$

2. Imaginary part of $\omega g^{*}$.

$$
\omega \xi\left(g^{*}\right)=G_{s} / G_{\infty}
$$


Real part and imaginary part of $\omega k^{*}$ are ignored if the material is incompressible.

Frequency $f$ is in radian per second.

In Equation (2.33) and Equation (2.34), $G_{\infty}$ is the long-term shear modulus. This data line is repeated as often as necessary to define the second, third, etc., terms in the Prony series. The number of terms in the Prony series is $\boldsymbol{N}$.

The vocal fold cover consists of a fluid-like material whose bulk modulus is similar to water, $B=2.2 \times 10^{8} \mathrm{~Pa}$. The amount of compression of water is assumed to be very small and can therefore be ignored. Hence, $\omega \Re\left(k^{*}\right), \omega \xi\left(k^{*}\right)$ are ignored in the material definition. Figure 2.12 and Figure 2.13 show the storage modulus $\left(G^{\prime}\right)$ and the loss modulus $\left(G^{\prime \prime}\right)$ measured by Chan and Titze [36].

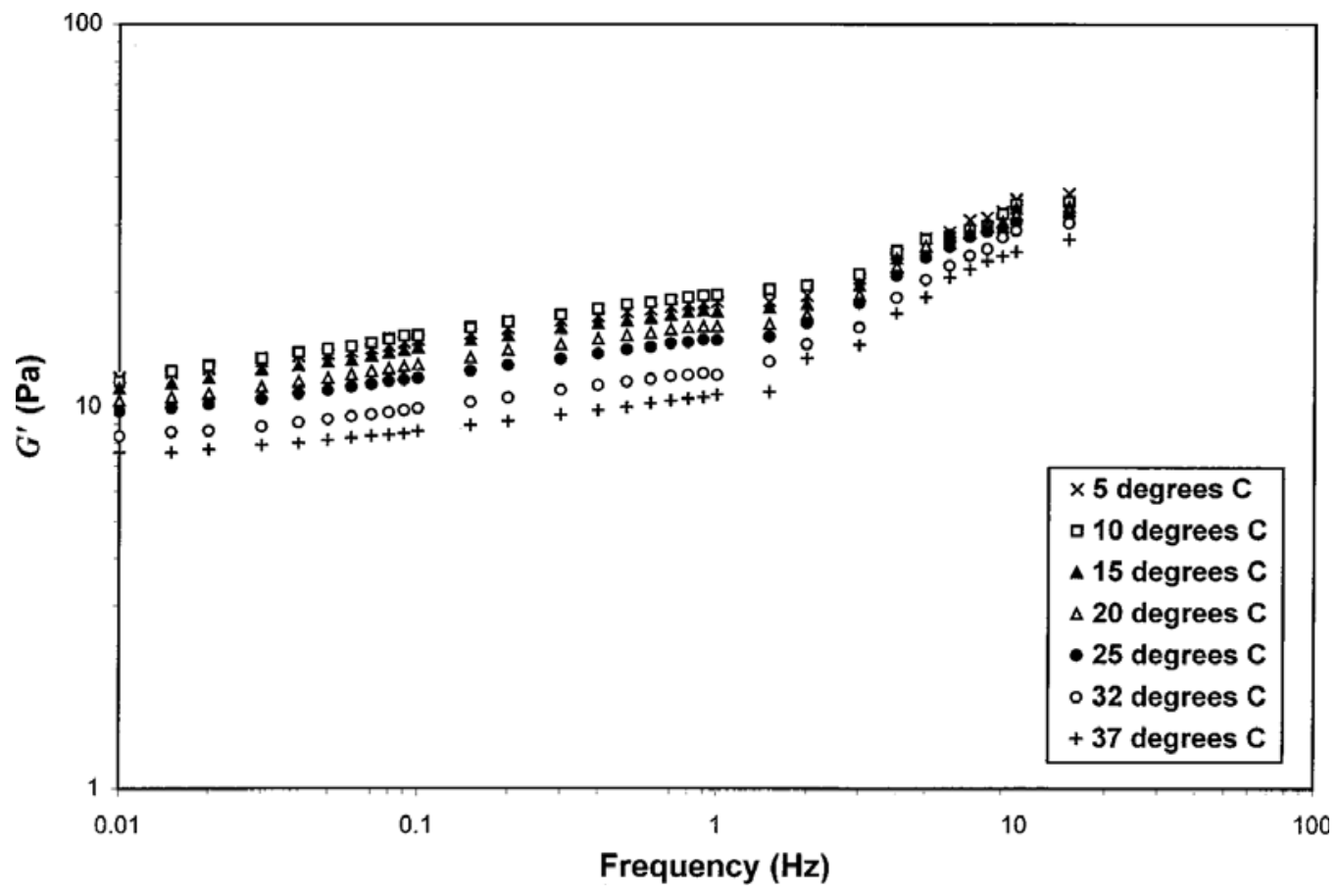

Figure 2.12 - Elastic shear modulus of the human vocal fold cover (59-year-old- male) as a function of frequency[36] 


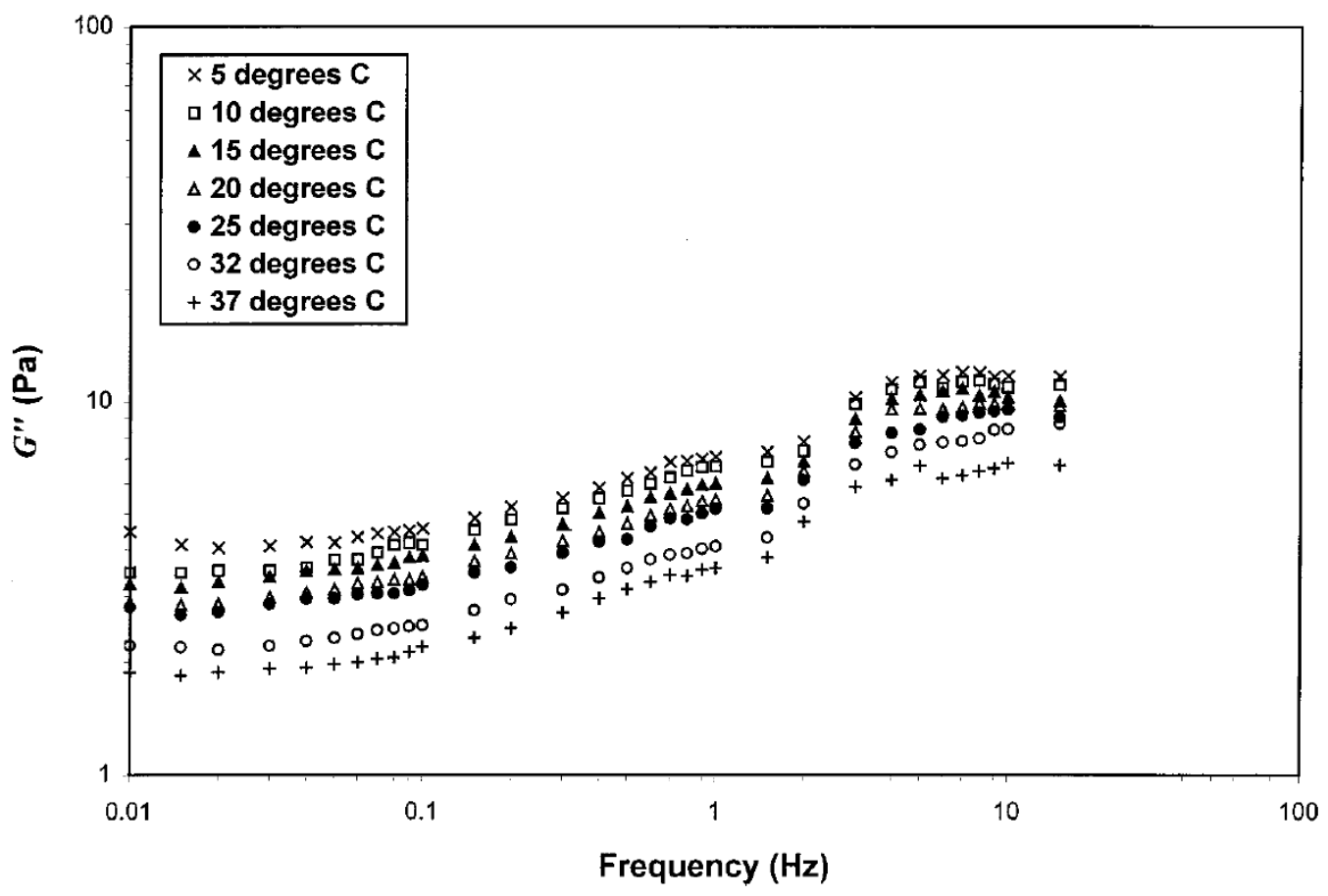

Figure 2.13 - Viscous shear modulus of the human vocal fold cover (59-year-old-male) as a fuction of frequency [36]

The data from Figure 2.12 and Figure 2.13 and calculated data for ABAQUS are displayed in Table AII.1.

\subsubsection{The Property of Body}

In a linear elastic continuum model, a transverse isotropic material in principle requires five independent tissue mechanical constants for the constitutive equations. These elastic constants include the longitudinal Young's modulus $E^{\prime}$ (an indication of tensile elasticity or stiffness along the direction of tissue fibres), the transverse and the longitudinal Poisson's ratios $v$ and $v^{\prime}$, respectively (an indication of tissue compressibility or the change of tissue volume upon deformation), and the transverse and longitudinal shear modulus $\mu$ and $\mu^{\prime}$.

The definition of transverse isotropy in ABAQUS uses the material editor: Mechanical $\longrightarrow$ Elasticity $\longrightarrow$ Elastic: Type: Engineering Constants

Transverse isotropy is characterized by a plane of isotropy at every point in the material. In this model, the $\mathrm{x}-\mathrm{z}$ plane is the plane of isotropy at every point, so transverse isotropy requires that 


$$
\begin{gathered}
E_{x}=E_{z}=E_{t}=E_{p} \\
v_{y x}=v_{y z}=v_{t p} \\
v_{x y}=v_{z y}=v_{p t} \\
G_{x y}=G_{z y}=G_{t}
\end{gathered}
$$

where $p$ and $t$ stand for "in-plane" and "transverse," respectively. Thus, $v_{t p}$ has the physical interpretation of the Poisson's ratio that characterizes the strain in the plane of isotropy resulting from stress normal to it. $v_{t p}$ characterizes the transverse strain in the direction normal to the plane of isotropy resulting from stress in the plane of isotropy. In general, the quantities $v_{p t}$ and $v_{t p}$ are not equal and are related by

$$
v_{t p} / E_{t}=v_{p t} / E_{p}
$$

The stress-strain laws reduce to

$$
\left\{\begin{array}{l}
\varepsilon_{x x} \\
\varepsilon_{y y} \\
\varepsilon_{z z} \\
\gamma_{x y} \\
\gamma_{x z} \\
\gamma_{y z}
\end{array}\right\}=\left[\begin{array}{cccccc}
1 / E_{p} & -v_{p} / E_{p} & -v_{t p} / E_{p} & 0 & 0 & 0 \\
-v_{p} / E_{p} & 1 / E_{p} & -v_{t p} / E_{p} & 0 & 0 & 0 \\
-v_{p t} / E_{p} & -v_{p t} / E_{p} & 1 / E_{t} & 0 & 0 & 0 \\
0 & 0 & 0 & 1 / G_{p} & 0 & 0 \\
0 & 0 & 0 & 0 & 1 / G_{t} & 0 \\
0 & 0 & 0 & 0 & 0 & 1 / G_{t}
\end{array}\right]\left\{\begin{array}{c}
\sigma_{x x} \\
\sigma_{y y} \\
\sigma_{z z} \\
\sigma_{x y} \\
\sigma_{x z} \\
\sigma_{y z}
\end{array}\right\}
$$

where $v_{p}$ is the Poisson's ratio in plane

$$
G_{p}=E_{p} / 2\left(1+v_{p}\right) \mu
$$

The parameters used in ABAQUS are listed as follows: transverse and longitudinal Poisson's ratios ( $v$ and $v^{\prime}$ ), transversal and longitudinal Young's modulus ( $E$ and $E^{\prime}$ ) and longitudinal shear modulus $\left(\mu^{\prime}\right)$.

As most parts of the human's body, the vocal folds contain more than 75\% water [40]. So they are considered to be nearly incompressible. To simplify the model, it is assumed to be $v=v^{\prime}=0.45$ which is standard value for incompressible materials. For 
low-strain, body tissue's Young's modulus is $E_{b}=20.7 \mathrm{kPa}$ [39]. As described in Equation (2.38), the parameters are calculated to be $E_{t}=E_{p}=E_{b}=20.7 \mathrm{kPa}$. The longitudinal shear modulus is defined $\mu^{\prime}=30 \mathrm{kPa}$ [22].

All the data used above agree with the present study: (1) For low strains, the value of $E^{\prime}$ (Young's modulus along y axis) for the body, cover and ligament are all on the order of $10^{5} \mathrm{dyn} / \mathrm{cm}^{2}$ (10000 Pa). (2) At low strains, the cover has about twice the stiffness of the body as estimated with the longitudinal Young's modulus [36].

\subsubsection{Define Step}

There are two types of analysis results required in this research. One is the frequency analysis and the other is dynamic analysis. They are defined separately and saved in different ABAQUS files.

1. Frequency analysis

In the step module, a new step is created with the procedure as following: Step $\longrightarrow$ Create $\longrightarrow$ Linear Perturbation $\longrightarrow$ Frequency. ABAQUS uses eigensolver to figure out the frequency problems. The matrix solver is chosen symmetric and the normalized eigenvectors are by displacement.

2. Dynamic analysis

Two steps are defined by the same process, Step $\longrightarrow$ Create $\rightarrow$ General $\longrightarrow$ Dynamic Explicit. The first step is 0.004 second, which is the process of air flow excitation. The seconds step is 0.015 second, which is the process of the model free vibration.

During the analysis of the mathematical model and physical model, Jiang [41] found that the liquid on a vibrating band tend to accumulate toward the midpoint of the band. Clinically, vocal fold nodules tend to occur at the mid-membranous fold. Therefore, it is more useful to figure out the dynamic response around the midpoint of the vocal folds. A small area is partitioned and selected as shown in Figure 2.14. The maximum glottal opening amplitude, closing velocity and contact stress are predicted occurring on the selected area. 


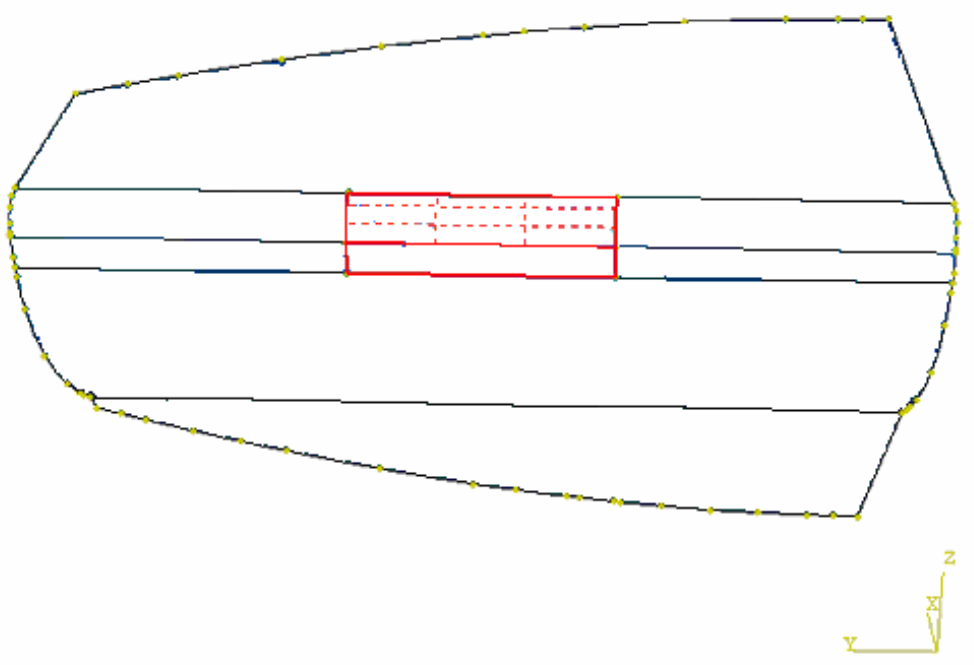

Figure 2.14 - Selected area in the middle of the vocal fold model

In the Field Output Request, the output variables are contact stress, contact normal force and stress components. In History Output Requests, the total contact area, total contact force, velocity and displacement of selected nodes are required.

\subsubsection{Assemble Model, Define Interaction, Load and Boundary Condition}

These steps are used to set the model's conditions as in the larynx. The two vocal folds are symmetrical about the mid-plane where they are set tangent at the top in the closed state. The contact area in the original position is assumed to be zero.

In real vocal folds, there is no obvious line of different layers. The air pressure, shear force will act on the whole organ. To make sure there is no opposite slide between the cover part and the body part during the vocal fold movement, it is necessary to define the constraint conditions between them. In ABAQUS, the constraint condition is defined as surface based tie which makes each of the nodes on the slave surface to have the same translational and rotational motion as well as the degrees of freedom as the point on the master surface to which it is closest. The adjust function is turned on to move the tied nodes on slave surface onto the master surface in the initial configuration, without any strain.

The medial surfaces of the vocal folds form a contact pair. Normally, the healthy vocal folds are covered with mucus as lubricant and the friction ratio between them is nearly zero. It is observed that there is little relative motion between the vocal folds while they 
touch each other. Therefore, interactive property between two vocal folds' surfaces is assumed to be frictionless. Furthermore, the contact forces on the medial surfaces are only normal to the surface and in medial-lateral direction.

The subglottal pressure $P_{s}$ leads to deformation of the vocal fold model during the simulation. A distributed load applied perpendicularly to the element faces that form the inferior and medial surfaces as shown in Figure 2.15. Several of the $P_{s}$ values are applied for various subglottal pressures. The static pressure $P_{s}=600,800,1000,1200$ and $1500 \mathrm{~Pa}$ are tested.

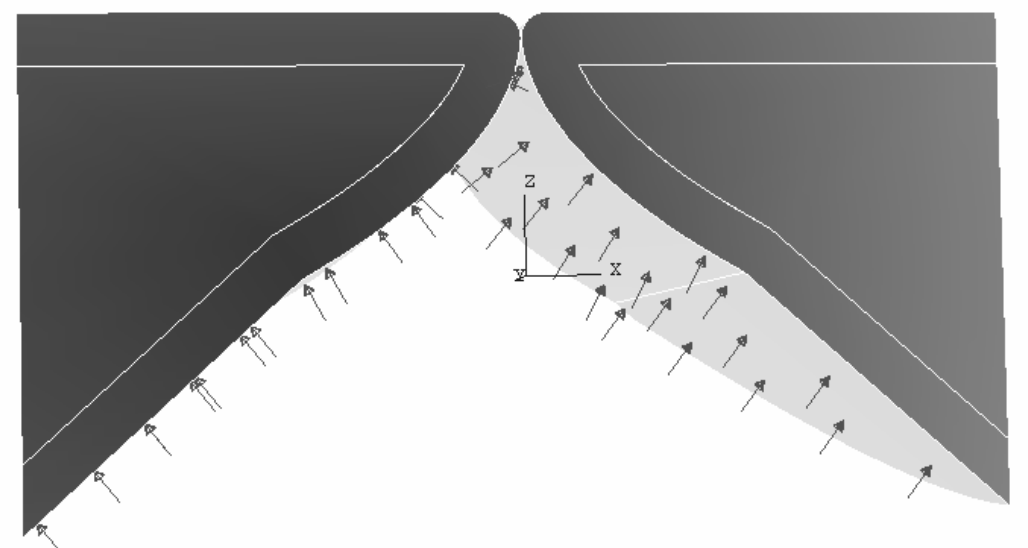

Figure 2.15 - Distributed load (due to the subglottal pressure) on the inferior surface

As discussed in Chapter 1, the vocal folds are fixed on arytenoids cartilages and thyroid cartilage in the larynx. Although the cartilages in the larynx should be flexible with a stiffness of $30 \mathrm{MPa}$, they are much stiffer than the vocal folds (stiffness of 20-40 kPa), and considered to be stationary in the analysis to simplify the algorithm. As shown in Figure 2.16, the anterior, posterior and lateral boundary conditions are fixed. Furthermore, the boundary conditions are defined as no movement and rotation in all directions $(\mathrm{U} 1=\mathrm{U} 2=\mathrm{U} 3=\mathrm{UR} 1=\mathrm{UR} 2=\mathrm{UR} 3=0)$. The vocal folds' superior and medial surfaces are allowed to move freely. 


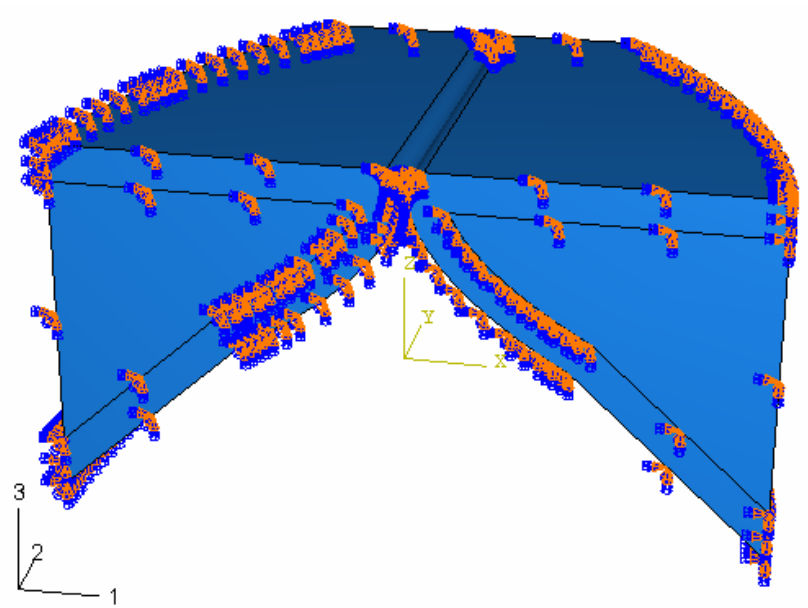

Figure 2.16 - Assembled model with defined boundary conditions

\subsubsection{Mesh}

The hex element is the best way to solve the finite element problems. However, the geometry of this model is nonlinear and it is hard for it to be meshed in that way. As discussed before, four nodes, a three-dimensional tetrahedral element is used to mesh the model. The layer part is divided by the element of $250 \mu \mathrm{m}$ which is 2 -fold fewer than the thinnest section of the layer part. The size of elements should be fine enough for the analysis. The element dimension of the body part is $0.5 \mathrm{~mm}$. The meshed model is shown in Figure 2.17.

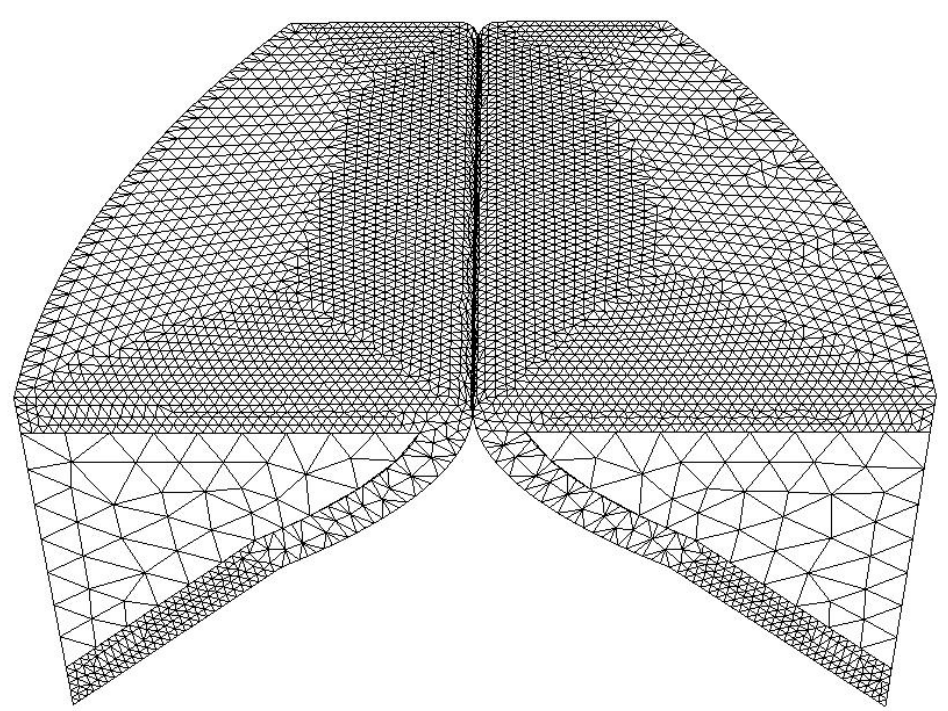

Figure 2.17 - Meshed by tetrahedral element (in ABAQUS)

The analyses were repeated by using a model with smaller mesh elements which were $100 \mu \mathrm{m}$ in the cover part and $200 \mu \mathrm{m}$ in the body part. The difference of impact stress 
between the two models is within $3 \%$, which indicates that the mesh is fine enough to achieve convergence of results.

\subsection{Closure}

Finite element analysis (FEA) of the ABAQUS is adopted in this work to link the relationship between the analytical and numerical modelling in the vocal folds structure while searching for the dynamic response in application to phonation process simulation. The model used the Titze and Talkin [30] geometry and viscoelastic material properties measured by Chan $[36,42]$. The simplification of the geometry has been proved to be possible and make it easy to build experimental model in the further research. The using of viscoelastic material will make the FEA model results more accurate.

In summery, numerical modelling can be a good approximation, depending on the chosen structure and material properties, to the analytical solution as shown in experimental data validation. 


\section{Chapter 3 EXPERIMENT INVESTIGATION}

\subsection{Introduction}

The main goal of this research is to use the finite element analysis to investigate the frequency characteristics and dynamic response of the vocal folds. Furthermore, experimental validation will enhance the results and also give them more validity. Clearly, it is much more accessible to do fundamental studies of the vocal folds by using a larynx model than by using human larynxes with invasive testing. Therefore, a physical model of larynx was fabricated to represent the human vocal folds in terms of the key parameters discussed in Chapter 2. This model has been constructed using a skin-like silicone casting and it exhibits a shape similar to the human larynx.

Two types of experiments were conducted. The first one used forced vibration to determine the frequency characteristics of the model and the second one used excitation by air flow to simulate the process of speaking. The purpose of this chapter is to confirm the accuracy of the FEA model described in Chapter 2 and to represent the vibratory deformation of the human vocal folds.

\subsection{Modal Analysis and Testing}

Modal analysis is a basic technique used to analyse many vibration structures. It is the process of using mathematical formulation for simulating the inherent dynamic characteristics of a system in terms of resonance frequencies, damping factors and mode shapes $[43,44]$. The limitation of modal analysis is that it is just available in linear systems. Modal testing is also called experimental modal analysis (EMA). In this method, the normal modes or frequencies of the vibrating system could be observed immediately after pulse excited, or during a sinusoidal force excitation. In practice, many of the nonlinear systems could be assumed approximately linear in small amplitude vibration. Compared to the finite element analysis (FEA) introduced in Chapter 2, EMA is based on experiment while FEA depends on computer analysis. 
EMA model is primarily performed to validate the accuracy of an FEA model. If an EMA model and an FEA model on the same structure both yield the same modes and the same natural frequencies, then it can be assumed that both could be accurately characterizing that structural dynamics. Once the FEA model has been validated, it can be used to simulate the vocal folds under various conditions.

A typical EMA MODEL may require a lot of measurements, and will give the results for many degrees-of-freedom (DOFs). In the human body, the vocal folds are fixed to the surrounding cartilages on three sides, so that the main freedom of the vocal folds is almost in the vertical direction. The three-dimensional diagram of the vocal fold is shown in Figure 2.1. However, for a single degree of freedom, the equation of motion may be written as [43]

$$
m \ddot{x}(t)+c \dot{x}(t)+k x(t)=f(t)
$$

Where $m, c, k, x$ and $t$ are defined as the mass, damping factor, stiffness, displacement and time respectively.

The Laplace transformation of this time domain equation is given by:

$$
X(s)=\frac{F(s)}{m s^{2}+c s+k}=G(s) F(s)
$$

To get the frequency response, $s$ is replaced by $j \omega$, where $\omega$ is the angular frequency (in radians/s) and $j$ is the imaginary number.

The theoretical basis of the modal analysis is the fact that the vibration responses at one location due to the excitation at the same or another location can be determined as a function of excitation frequency. This relationship is called frequency response function (FRF). The vibration response is a set of simple harmonic motions and the natural frequencies resulting from the linear time-invariant dynamic system are the Fourier series of the functions. 


\subsection{Experimental Set-up}

The two experimental setups used in the research are shown in Figure 3.1. Figure 3.1-a shows the forced vibration experimental set-up. The setup consists of an electromagnetic shaker which is driven by a signal generator through a power amplifier. The response is picked up by a Polytec OFV505 laser which is connected to a Polytec OFV505 acquisition system. The output signal is displayed on a TDS1012 oscilloscope and a PC. The first setup is used in determining the forced response.

The second setup, in Figure 3.1-b, is used to determine the vocal folds deformation due to speaking. The larynx model is driven by an air flow while the signal is picked up by the laser detector as in the first experiment.

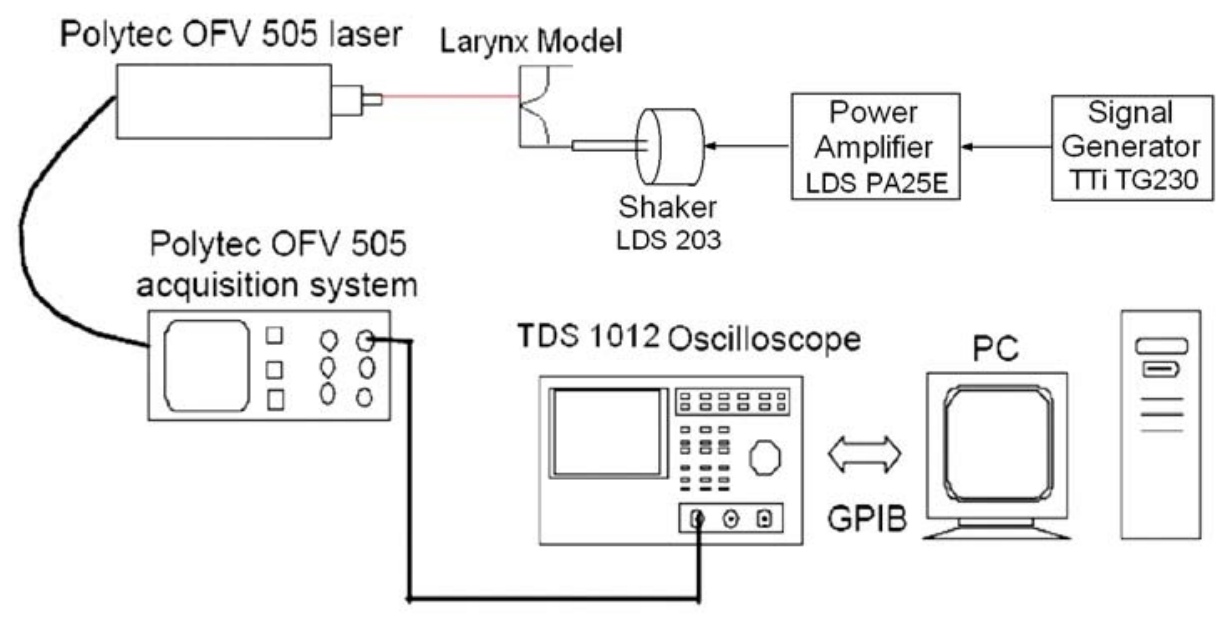

a

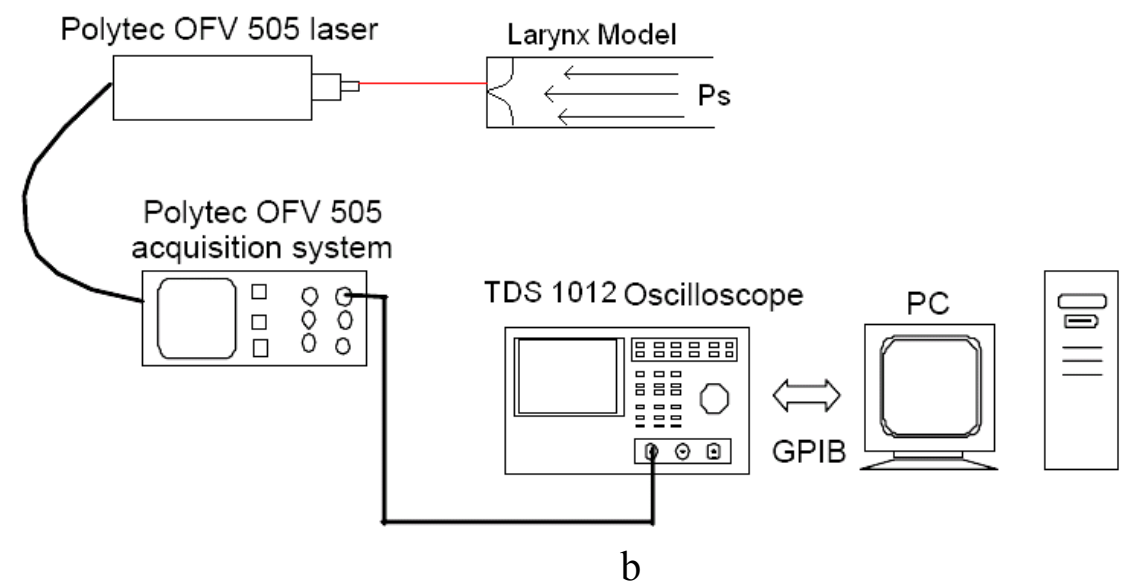

Figure 3.1 - (a) A block diagram of the forced vibration experimental set-up (b) A block diagram of the second experimental set-up 
The results from both experiments will be compared with the theoretical results from the ABAQUS model and discussed in the next chapter.

\subsection{Physical Model}

The physical model is designed to simulate three key parameters of the human vocal folds:

(1) the mechanical properties (including density, stiffness and viscosity);

(2) geometry (including dimensions and shape), also known as the static, undeformed shape; and

(3) the boundary condition, fixed with unmovable cartilage.

\subsubsection{Vocal Folds Model}

Human tissues are soft, extensible and nearly incompressible [45]. Since the 1960s, silicone has been introduced into many biomedical application and today is one of the most used and important biomaterials [46]. A two-component liquid silicone is used to make the vocal fold models which are cast by rigid moulds. The rigid moulds were made of polymer clay, which is both easy to shape and accessible. The general size of the male vocal fold is approximately $1.4 \mathrm{~cm} \times 1 \mathrm{~cm} \times 1 \mathrm{~cm}$ [30]. In order to show the dynamic response of the model clearly, the model is enlarged to be double the size of a realistic vocal fold. The model shape is an idealistic vocal fold based on the geometry described in Chapter 2.

The vocal folds model is made from silicone PROSIL $8^{\circledR}$ which is classified to be prosthetic grade RTV silicone. It is very soft, skin safe silicone for use in prosthetic and orthopaedic applications [47]. The mixing ratio of part A and part B of PROSIL $8^{\circledR}$ is $2: 1$. Before pouring the mixture into the moulds, the inter-surfaces of the moulds were coated with a thin layer of silicone lubrication to smooth the model's surface and to make it easier to remove the model from the moulds. The curing process took approximately about 8 hours and the environmental temperature kept around $30^{\circ} \mathrm{C}$. After curing, the silicone was removed from the mould. The silicone model of the left side vocal fold is shown in Figure 3.2. 


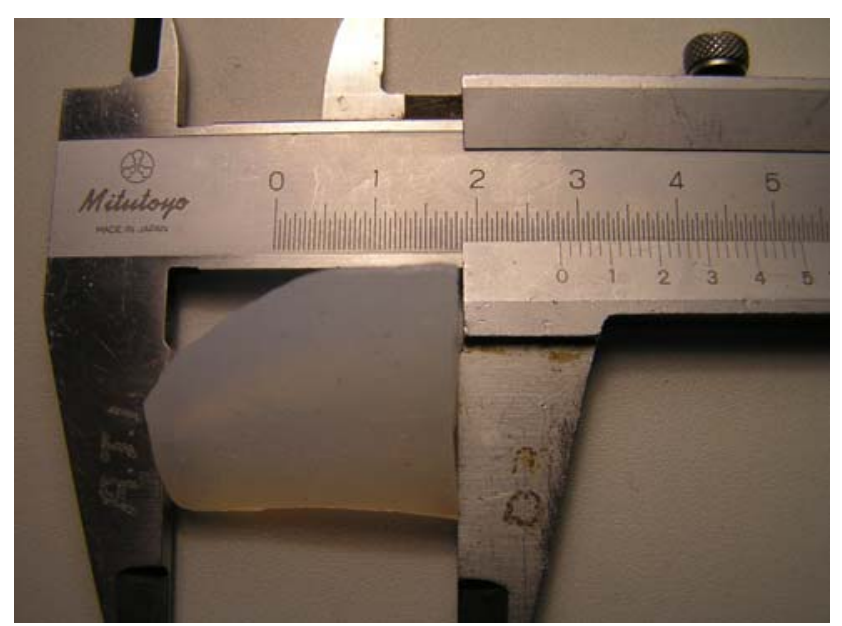

Figure 3.2 - The silicone model of the left side vocal fold

\subsubsection{Material Testing}

The parameters of cured silicone are listed in the product handbook [47]. The key parameters are: density $1.08 \mathrm{~g} / \mathrm{cm}^{3}$, hardness $8 \mathrm{~A}$ (in SI unit, about $50 \mathrm{kPa}$, very flexible, floppy, and similar to skin), and viscosity $1.5 \mathrm{~Pa} \cdot \mathrm{s}$ (15 poise). All of the parameters are quite near to human vocal folds' mechanical parameters: density $1.043 \mathrm{~g} / \mathrm{cm}^{3}$, hardness $40.7 \mathrm{kPa}$, viscosity $0.8 \mathrm{~Pa} \cdot \mathrm{s}[31,39,48]$. However, as shown in most of the literature, human tissues are usually nonlinear materials with response dependent on the strain.

The stress-strain curve is a good way to describe the elasticity of the material. It is a graph derived by measuring the load (stress, $\sigma$ ) versus extension (strain, $\varepsilon$ ) for a sample of a material. The male vocal folds' stress-strain curves are shown in Figure 3.3 (curve M1L, the highlighted curve) and Figure $3.4[39,48]$.

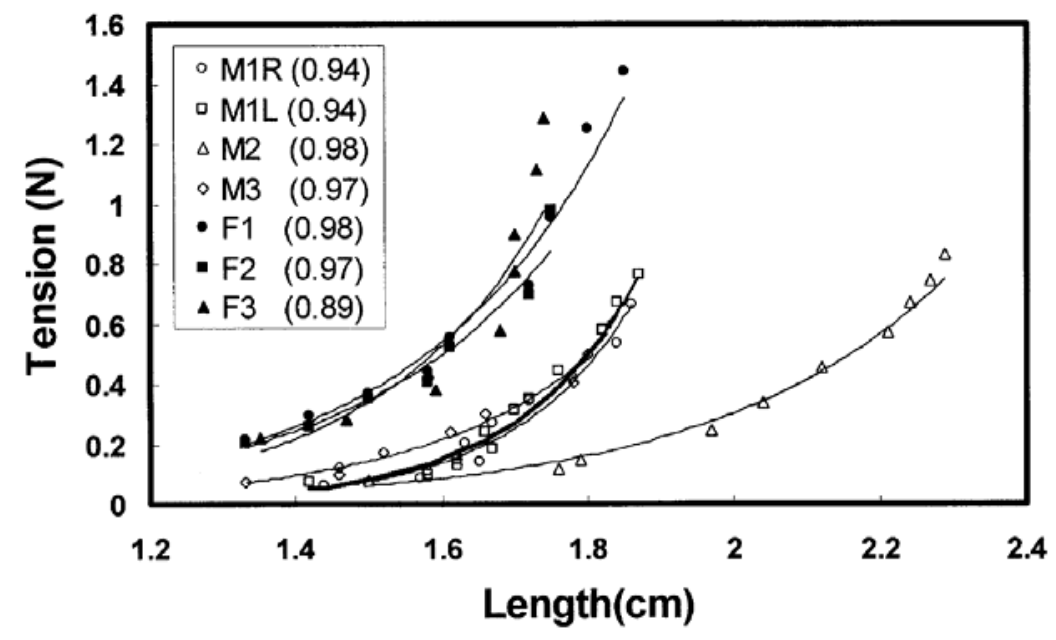

Figure 3.3 - Stress-strain curves with the vocal fold length of the lowest vibration frequency as a reference. $M 1 L=$ man \#1, the left vocal fold [48] 


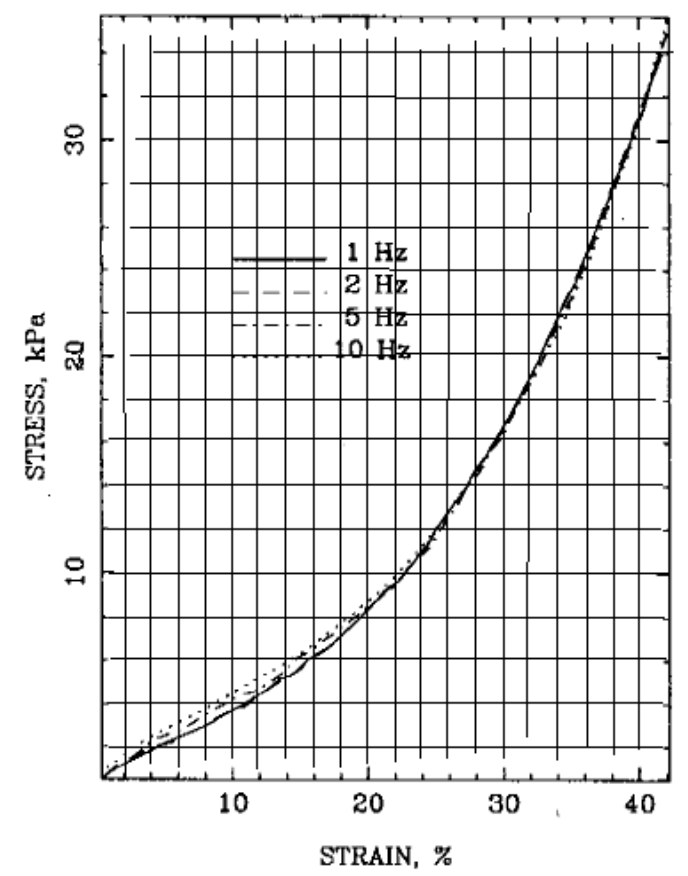

Figure 3.4 - Stress-strain curves of a sample of vocal fold cover tissue stretched and released from high to low frequencies [39]

To calculate the stress-strain relationship for silicone PROSIL $8^{\circledR}$, a material testing machine (Tinius Olson HK505-S, Figure 3.5-a) was used. Five silicone PROSIL $8^{\circledR}$ beam samples were prepared for the force and extension test. The silicone beams were tested by stretching them within the strain of $100 \%$ before they were broken.

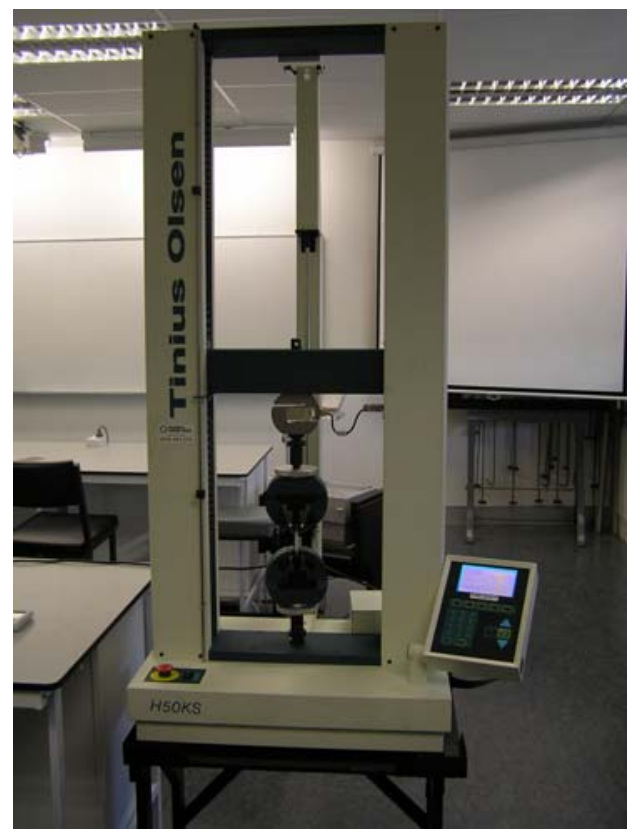

a

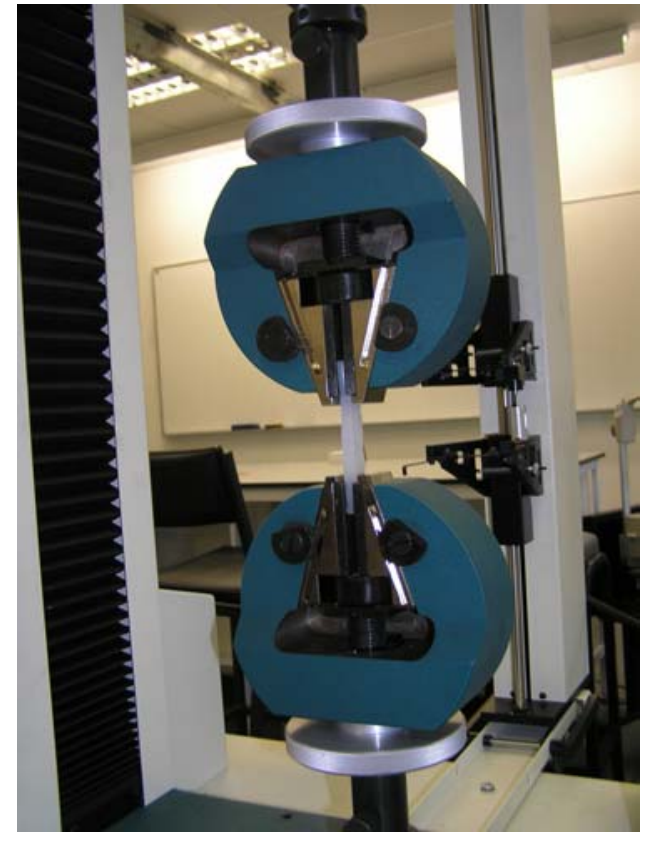

b

Figure 3.5 - HK505-S benchtop material testing machine 
Before the stress-strain test, all of the specimens were preconditioned. They were subjected to 20 tension-compression cycles to a strain amplitude of 0.5 at a frequency of $0.1 \mathrm{~Hz}$. Their rebound to the original (unstrained) condition between two load cycles was very small. The results illustrated that the internal structure of the silicone specimens were stable.

The viscoelastic material is usually strain rate sensitive. The loading speed of the test was started from $20 \mathrm{~mm} / \mathrm{min}$ and then changed to $50 \mathrm{~mm} / \mathrm{min}$. It was found that this changed the loading force slightly. Thus, the silicone material can be regarded as being insensitive to the used strain rate.

As shown in Figure 3.5-b, the samples were placed between the holders and firmly clamped at both ends. The lower holder was fixed while the upper holder was connected to a motor which was driven at preset speed. The upper holder was connected to a force sensor with an accuracy of $0.1 \mathrm{~N}$. During the stretching process, the force sensor transferred measured force to a display. The force and extension reports data are given in Appendix III. The force and extension data were converted to stress and stain and shown in Table AIII 1-5. The stress $(\sigma)$ is obtained by dividing the load by the original area of the cross section of the sample and the strain $(\varepsilon)$ is obtained by dividing the elongation of the sample by the original length of the sample.

$$
\begin{gathered}
\sigma=\frac{F}{A}\left[\frac{N}{m^{2}}\right] \\
\varepsilon=\frac{\Delta l}{\Delta l_{0}}
\end{gathered}
$$

The stress-strain curve based on the test data is shown in Figure 3.6. The slope of the stress-strain curve in the elastic region is known as material's Young's Modulus $E$ which is the ratio of the stress to the strain. 


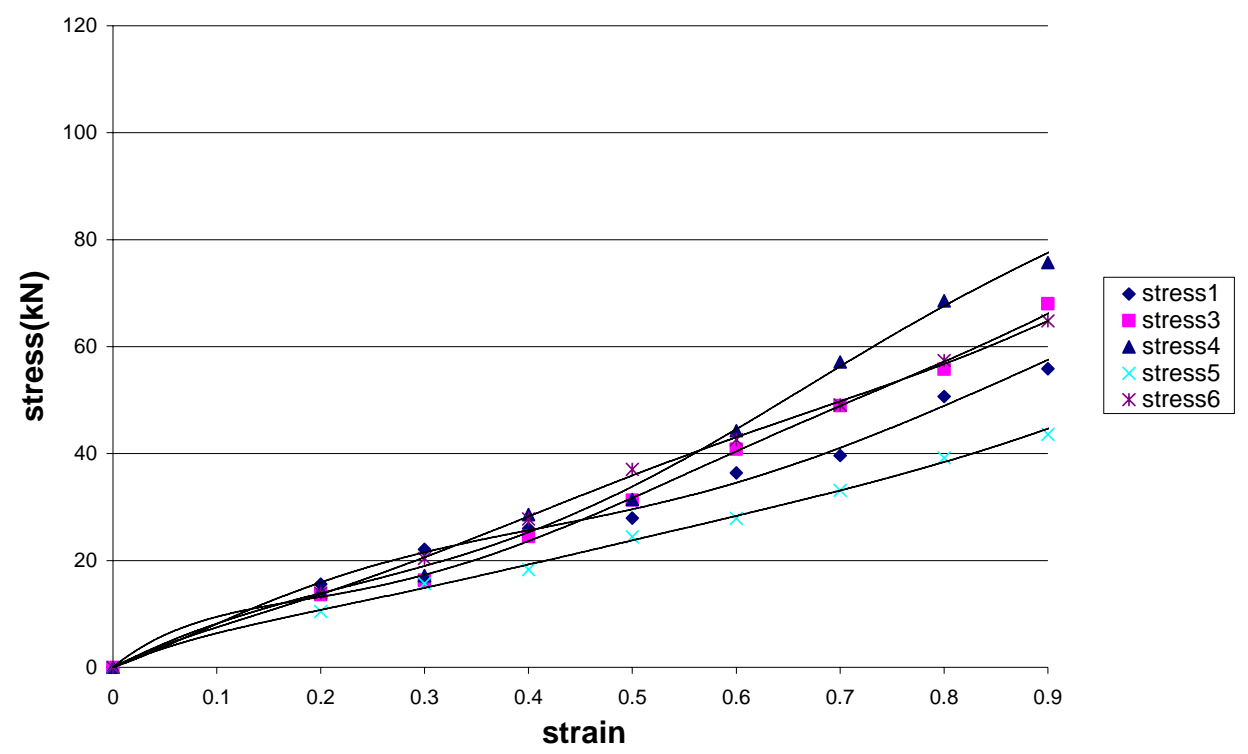

Figure 3.6 - Silicone elasticity and strain test data

Comparing Figure 3.6 with Figure 3.3 and Figure 3.4, it is clear that the elasticity (Young's modulus) of silicone PROSIL $8^{\circledR}$ is similar to the human vocal fold in vitro throughout the entire strain range.

Another key parameter of the vocal folds is the viscosity. The test data of the vocal fold is 5-8 Poise [31, 36] and the viscosity of silicone PROSIL $8^{\circledR}$ is about 15 Poise [47]. This will result in the silicone having a slightly longer response delay than the human vocal fold in vivo testing. However, the difference of the two materials will not affect the final results significantly, and can be ignored. In conclusion, the silicone PROSIL $8^{\circledR}$ is a suitable material to represent the vocal folds tissue.

\subsubsection{Boundary Conditions}

A larynx frame was made to place the silicone vocal folds as shown in Figure 3.7. The anterior-posterior ends and lateral surface of the models were attached to a rigid clay frame using a liquid adhesive (super glue). In the frame, the two silicone vocal folds are symmetric about the mid-plane and are tangent at the top in the closed state. The surface of the polymer was coated with a thin layer of talc to reduce the surface tackiness. Before fixing the frame into the duct, the vocal folds model was tested in a forced vibration experiment to determine that they are rigid enough to resemble clamped support. 


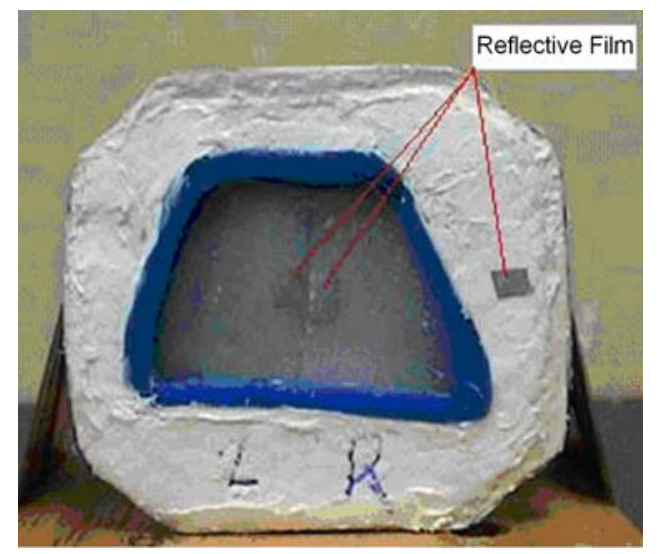

Figure 3.7 - The silicone vocal folds model fixed in the cartilage mould

\subsubsection{Larynx Model}

The physical larynx model was situated in a rectangular duct (wind tunnel). The edges between the frame and the wind duct were sealed so that the supplied air only flowed through the glottal between the two silicone vocal folds. This is different from the anatomical structure shown in Figure 1.2, where the trachea's diameter is about $1.4 \mathrm{~cm}$ [49]. The purpose of the wind tunnel is to supply stable and uniform air pressure to the vocal fold models and to sustain the model oscillation. Therefore the wind tunnel is more than three times larger than the normal size in order to supply stable air pressure.

\subsection{Experimental Equipment}

Referring to Figure 3.1, the two experimental setups still require a detection system and a vibration system.

\subsubsection{Vibration System}

The vibration system consists of: electrodynamic shaker, signal generator and power amplifier.

The electrodynamic shaker (Ling Dynamic Systems Ltd., LDS V203, shown in Figure 3.8-a) is a permanent magnet electrodynamic shaker designed for vibration test applications including modal and structural analysis. The shaker is driven by a power amplifier at constant excitation power. 
The power amplifier (LDS PA25E, shown in Figure 3.8-b) was designed to operate with support LDS vibration test systems that use permanent magnet shakers which use separate field power supplies.

The signal generator (TTi TG230, shown in Figure 3.8-c) offers all of the standard generator functions including sine, square, triangle waveforms, variable DC offset, and variable symmetry output. An additional internal sweep generator with linear or log sweep and a variable sweep rate is also provided.

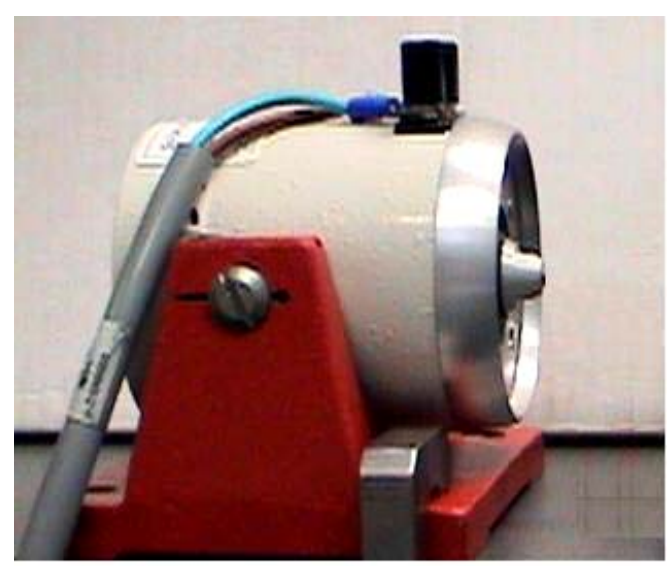

a

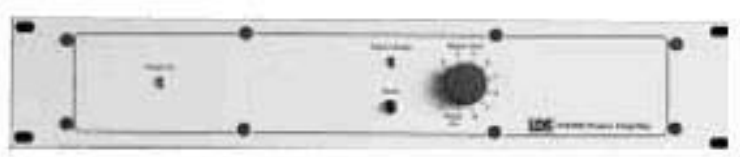

b

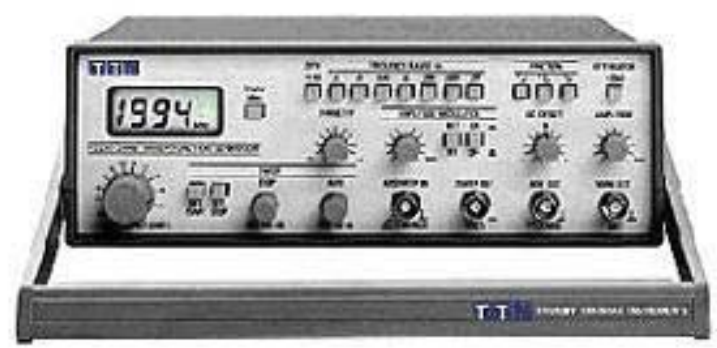

c

Figure 3.8 - Equipment of vibration system

\subsubsection{Detection System}

The detection system includes a laser sensor, vibrometer controller, computer and oscilloscope.

Due to the small size of the model and the softness of the material, if any contact sensors are used they will cause some errors. Non-contact sensing is a better choice for the test. A vibrometer Polytec OFV-505 coupled to an OFV-5000 modular controller acquires data by measuring back-scattered laser light from a reflective paper based on the Doppler principle (Figure 3.9-a). It offers achievable velocity range of 0-1000 $(\mathrm{mm} / \mathrm{s}) / \mathrm{V}$. For accurate measurement, the best distance between the laser sensor and the measured point is approximate $30 \mathrm{~cm}$ and with a vibrometer warm-up time of at least 20 minutes for proper operation.

The output signal was sent from the OFV-5000 modular vibrometer controller to a Tektronix digital storage oscilloscope (TDS 1012) as shown in Figure 3.9-b. This 
oscilloscope has a $200 \mathrm{MHz}$ bandwidth and a $2 \mathrm{GS} / \mathrm{s}$ sample rate and was used to observe the dynamic response during closure. This data was then transferred to a PC.

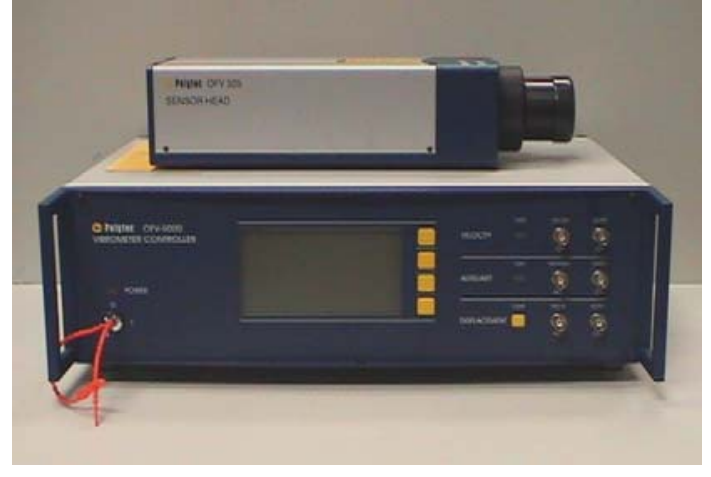

a

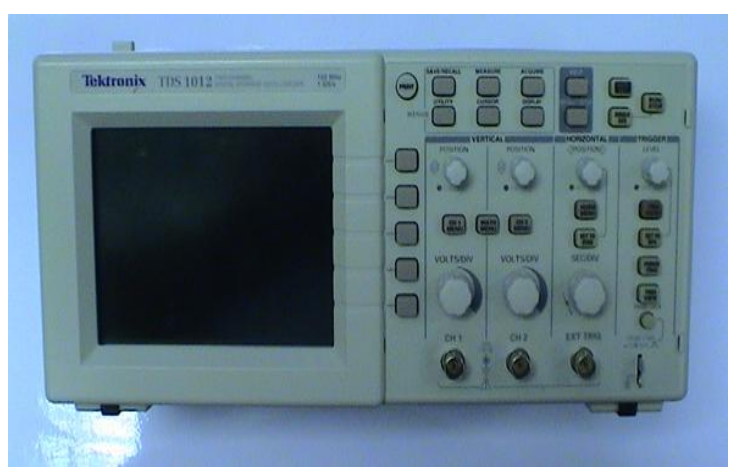

b

Figure 3.9 - Laser sensor head, vibrometer controller and oscilloscope

\subsection{Experimental Procedures}

Forced vibration test and air flow excitation test are introduced to find out the EMA model's frequency characteristics and dynamic response.

\subsubsection{Forced Vibration}

Forced vibration is the vibration that takes place under the excitation of external forces. When the excitation is oscillatory, the system is forced to vibrate at the excitation frequency. If the frequency of excitation coincides with one of the natural frequencies of the system, a condition of resonance is encountered.

As shown in Figure 3.10, the model was clamped in a vice which was attached to a rigid surface. The electrodynamic shaker (LDS V203) with an extended pointer was placed in contact with the sample at one end and acted as a vibration exciter. The shaker pointer was positioned just touching the sample location and the vibration amplitude was kept very small to avoid "over-driving" the sample. The excited amplitude of the shaker was controlled by the power amplifier (LDS PA25E). The excited frequency of the shaker was driven by the signal generator (TTi TG230). The predicted dominant resonance frequencies of the model were between 50 and $180 \mathrm{~Hz}$. Therefore, the frequency range of TTi TG230 was set $0 \sim 200 \mathrm{~Hz}$. Sinusoidal signal of constant input power was delivered to the shaker and the frequency was linearly increased at a sweep rate of 20 $\mathrm{Hz} / \mathrm{second}$. 
As demonstrated previously (see section 3.5.1), the vibration amplitude varied at different frequencies during the sweep process. Furthermore, it was observed that the amplitude changed slightly and decreased smoothly during the sweep process. Therefore, the change of shaker vibration amplitude is ignored in this experiment.

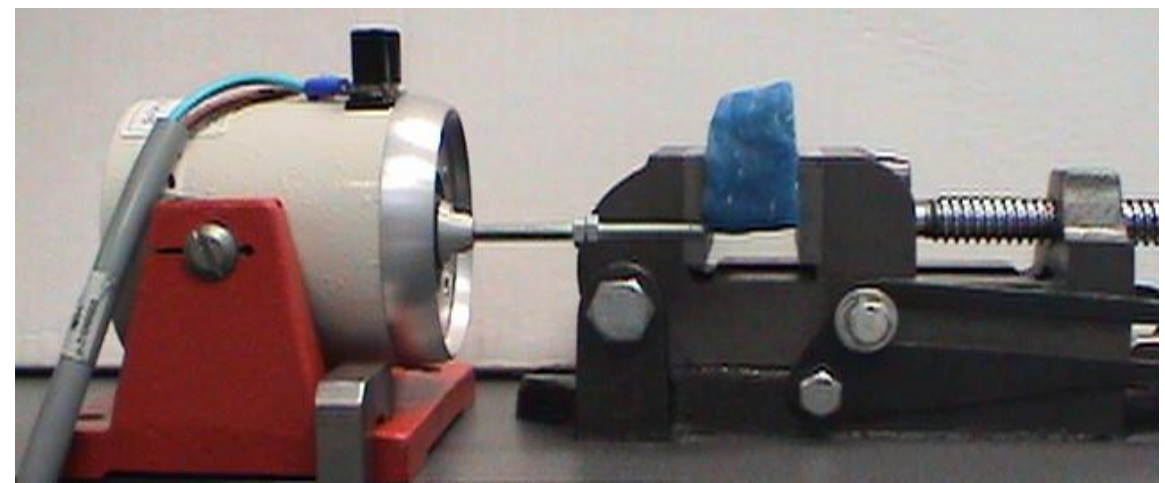

Figure 3.10 - Photo of the position of the shaker and the model

As shown in Figure 3.7, the measured points were on the middle of the vocal fold model, which were assumed to present maximum deformation. Because the model size and amplitude of forced vibration was very small, the sensitivity of the sensor was set to maximal $\sim 5(\mathrm{~mm} / \mathrm{s}) / \mathrm{V}$. During the excitation process, the movement of the measured point was converted into a voltage signal and displayed on the screen of the oscilloscope (TDS 1012). The oscilloscope recorded the results and displayed the frequency domain data using a Fast Fourier Transform (FFT). FFT analysis is the most common method to calculate the RFR in modal analysis. In this process, two peaks were detected, which show the resonance frequencies of the model. The saved data was then transferred to a computer for analysis.

Since at a resonance frequency, the motion may grow to quite large values, the vibration frequency was controlled manually and set to approximately the resonance frequency found from last step to confirm the resonance frequency found through FFT data. The time domain data at different frequencies was saved and compared to previously acquired data.

The experiment was repeated several times and both sides of the model were tested to ensure the accuracy of the results. 


\subsubsection{Air Flow Excitation}

In this experiment, an impulse input is introduced and the closure process occurs in less than $10 \mathrm{~ms}$, which is beyond the capabilities of the oscilloscope. Therefore, the waveform was captured and recorded using LabVIEW ${ }^{\mathrm{TM}}$ software which is a graphical software system for developing high-performance scientific and engineering applications. The connection between the oscilloscope and the PC was via RS-232 standard plug-in I/O board - NI-DAQ7. The output data was saved in a text file which was transferred into Microsoft Excel $^{\mathrm{TM}}$ for further data processing and analysis.

A new LabVIEW file was created to record the data as show in Figure 3.11. In this file, channel 4 was chosen as the I/O port. The scan rate was set $40000 \mathrm{scans} / \mathrm{sec}$ to make sure most of the motion would be recorded.

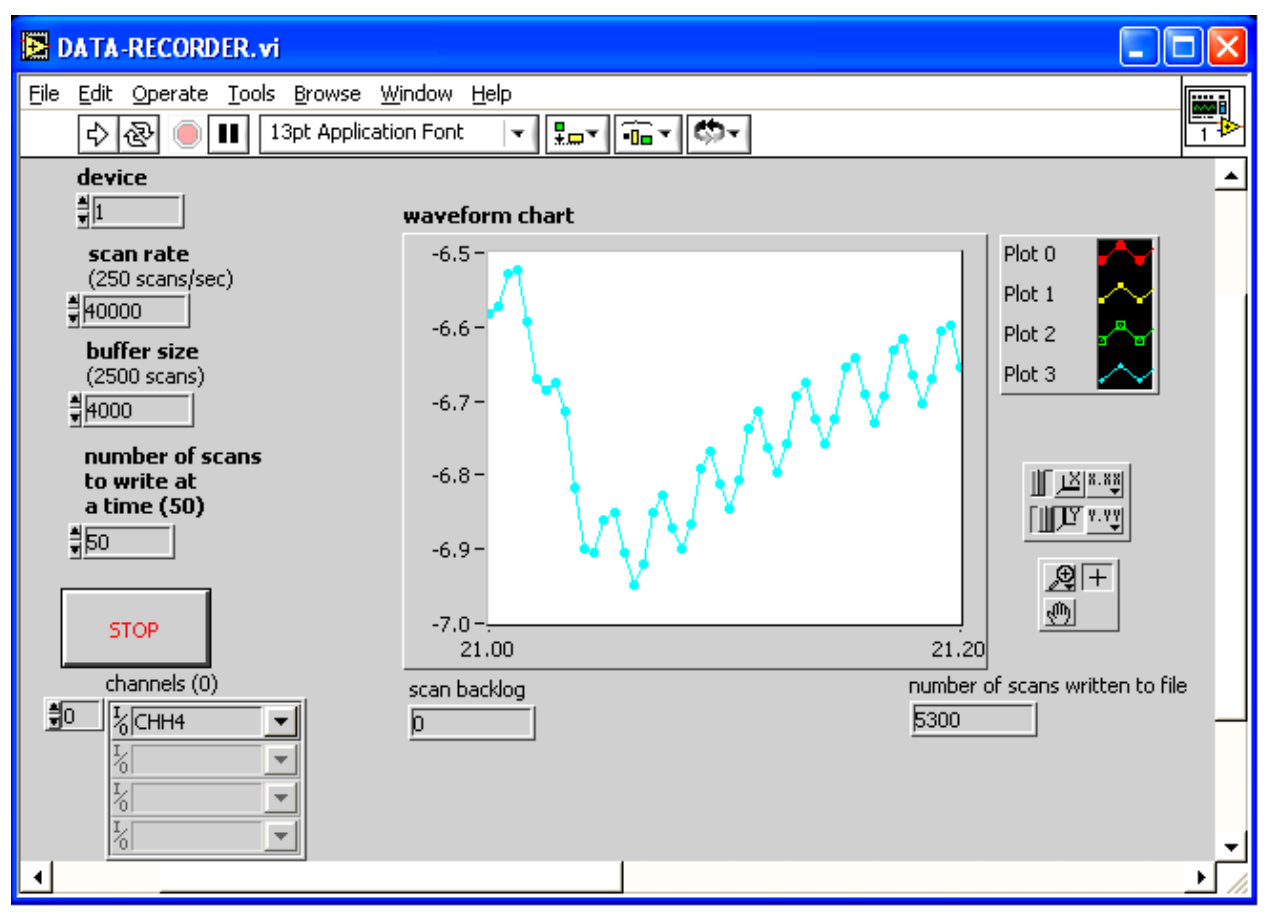

Figure 3.11 - LabVIEW file

As shown in Figure 3.12, the larynx model was fixed on a board which was clamped on the table and the laser sensor (Polytec OFV-505) was placed approx $30 \mathrm{~cm}$ from the larynx model. On the other side of the wind duct, the larynx model was connected to an air pipe which supplied static air pressure to the wind duct.

The supplied air pressure was simulated in a step input as shown in Figure 3.13. Initially, the air pressure was at $3000 \mathrm{~Pa}$, defined as the threshold pressure. After a few seconds, 
due to material damping, the model became static. The air flow supply was then ceased, and the vocal folds model started to close.

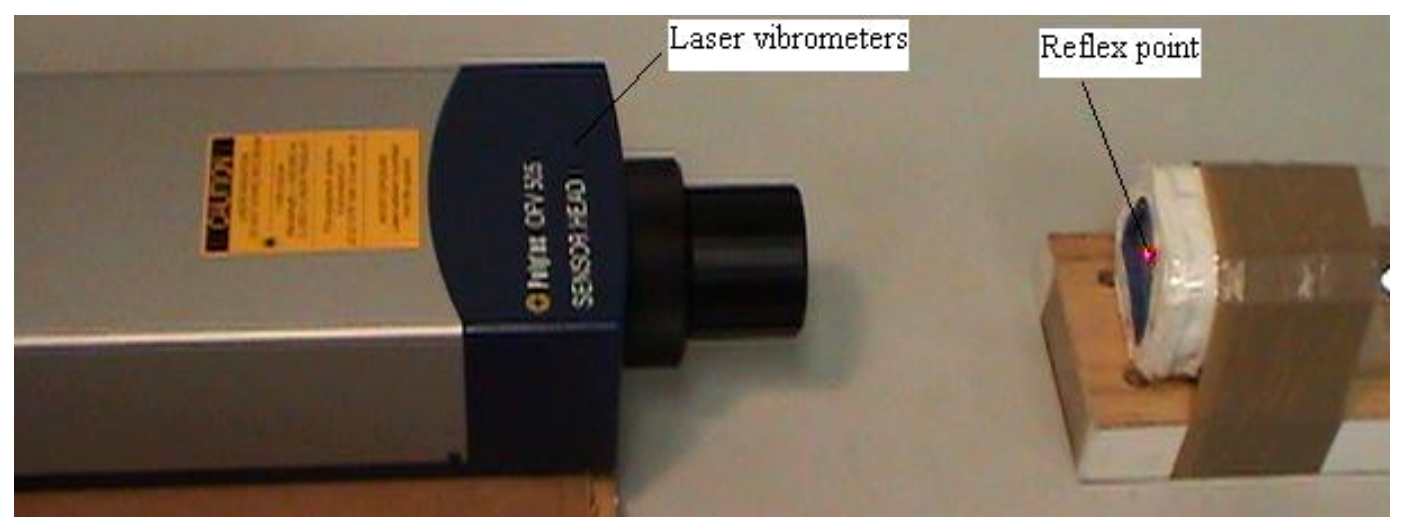

Figure 3.12 - Photo of the position of the laser head and larynx model

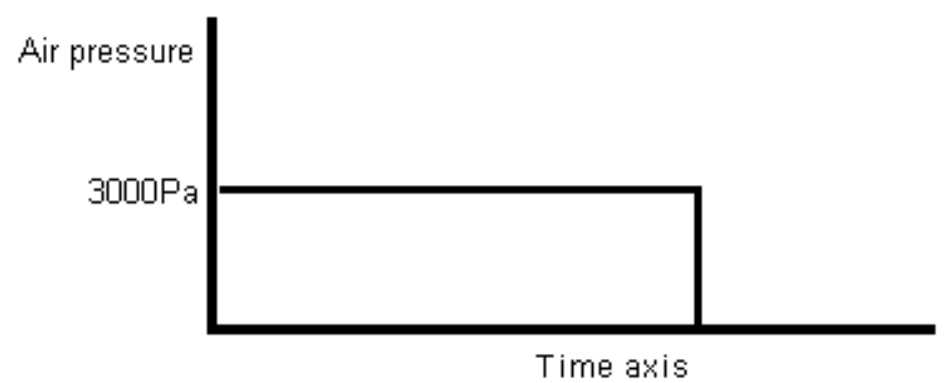

Figure 3.13 - Air flow supply process

It was supposed that the maximum velocity of the midpoint would be approximately $100 \mathrm{~mm} / \mathrm{s}$. Therefore, the measurement sensitivity of modular vibrometer controller was set to be $25(\mathrm{~mm} / \mathrm{s}) / \mathrm{V}$ with the voltage output range from $-5 \mathrm{~V}$ to $+5 \mathrm{~V}$. Furthermore, the maximum frequency was set to $1.5 \mathrm{MHz}$ in order to capture as much data as possible during the closure. The settings on the TDS 1012 oscilloscope were set to 1 $\mathrm{V} /$ division on the $\mathrm{Y}$-axis and $100 \mathrm{~ms} /$ division on the $\mathrm{X}$-axis. For each experiment, data was acquired as soon as the pressure was reduced until the vocal folds model in static state.

Figure 3.7 shows positions of reflective paper on the model. Two pieces of reflex paper were placed on the midpoints of both sides of the vocal folds to obtain the vocal fold's response result. Another piece of reflex paper was placed on the edge of the model to get the response of rigid part in the larynx model and to ensure that the vibration of the whole model wouldn't produce significant noise and affect the final results of the experiment. 


\subsection{Summary}

This chapter introduced a new larynx model used to validate the FEA. Due to equipment being unavailable, measurements were made only to determine the natural frequencies and velocity in the experiment. No attempt was made to measure the mode shape in frequency test and deformation shape in the phonation simulation process. The results of the experiment will be presented in Chapter 4 and compared with theoretical results. 


\section{Chapter 4 RESULTS}

\subsection{Introduction}

This chapter presents the frequency and dynamic results of the vocal folds analysis. Both theoretical model and experimental model are considered. For the experimental model, the resonance frequencies and dynamic response are obtained by forced vibration and step input. For theoretical model, the results are calculated using ABAQUS software.

\subsection{Experimental Results}

All of the test data were exported to Microsoft Excel, and shown in a graphical format for easier inspection.

\subsubsection{Forced Vibration Results}

The main purpose of the experiment was to determine the resonance frequencies of the experimental model, at which the vocal folds exhibit maximal amplitudes of vibration. When the excited vibration was swept from $10 \mathrm{~Hz}$ to $200 \mathrm{~Hz}$, the output results were displayed in the frequency domain using the Fast Fourier Transformation (FFT) mode. The peaks observed on the oscilloscope screen demonstrate the resonance frequencies.

Exported from the oscilloscope, Figure 4.1 and Figure 4.2 show samples of the graphs of vibration intensity versus frequency of the midpoint of the vocal fold model. The two peaks of the left vocal fold element are $75 \mathrm{~Hz}$ and $131 \mathrm{~Hz}$. However, in the right vocal fold element, they are $79 \mathrm{~Hz}$ and $133 \mathrm{~Hz}$. 


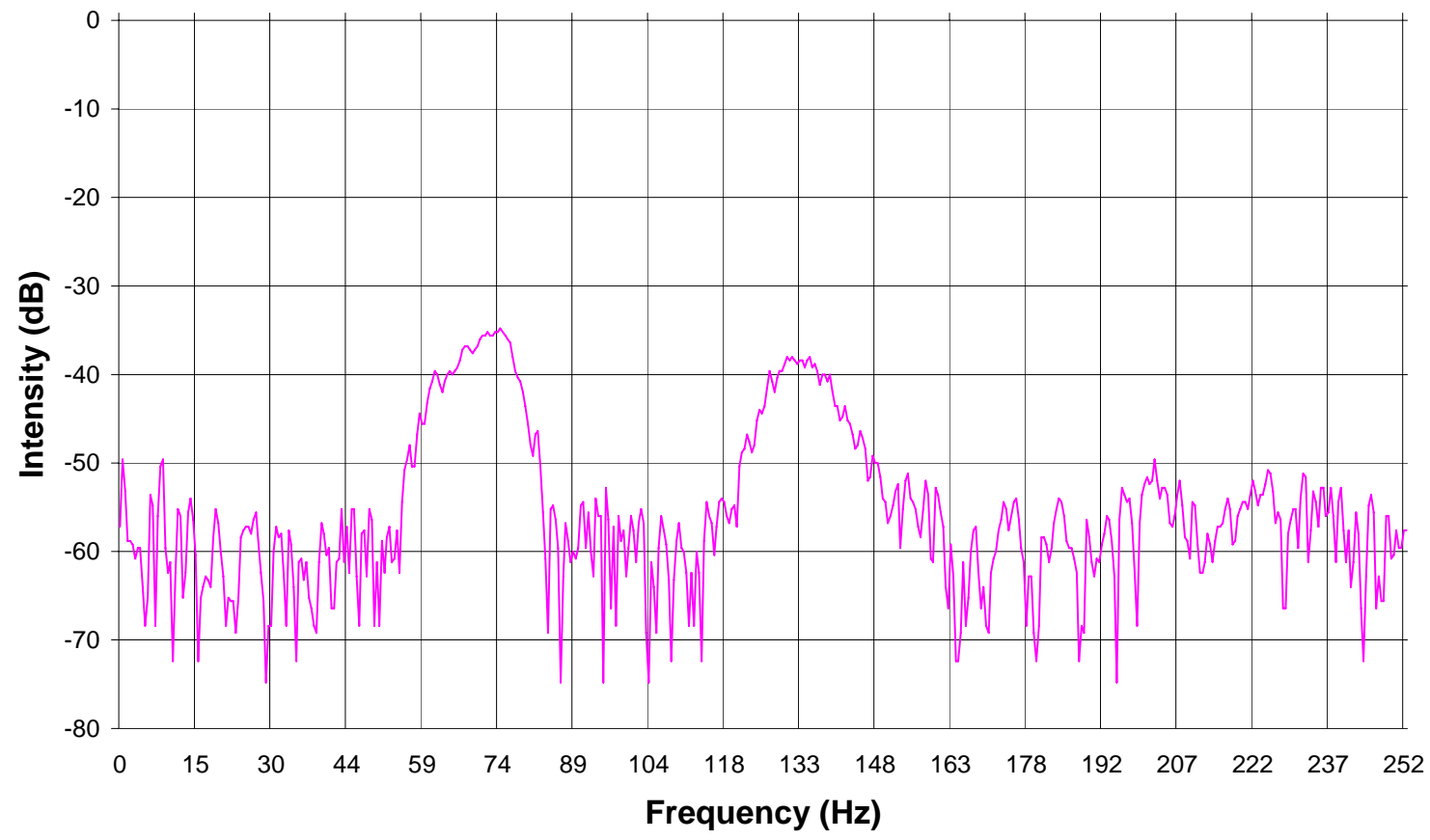

Figure 4.1 - Frequency response of the left vocal fold element

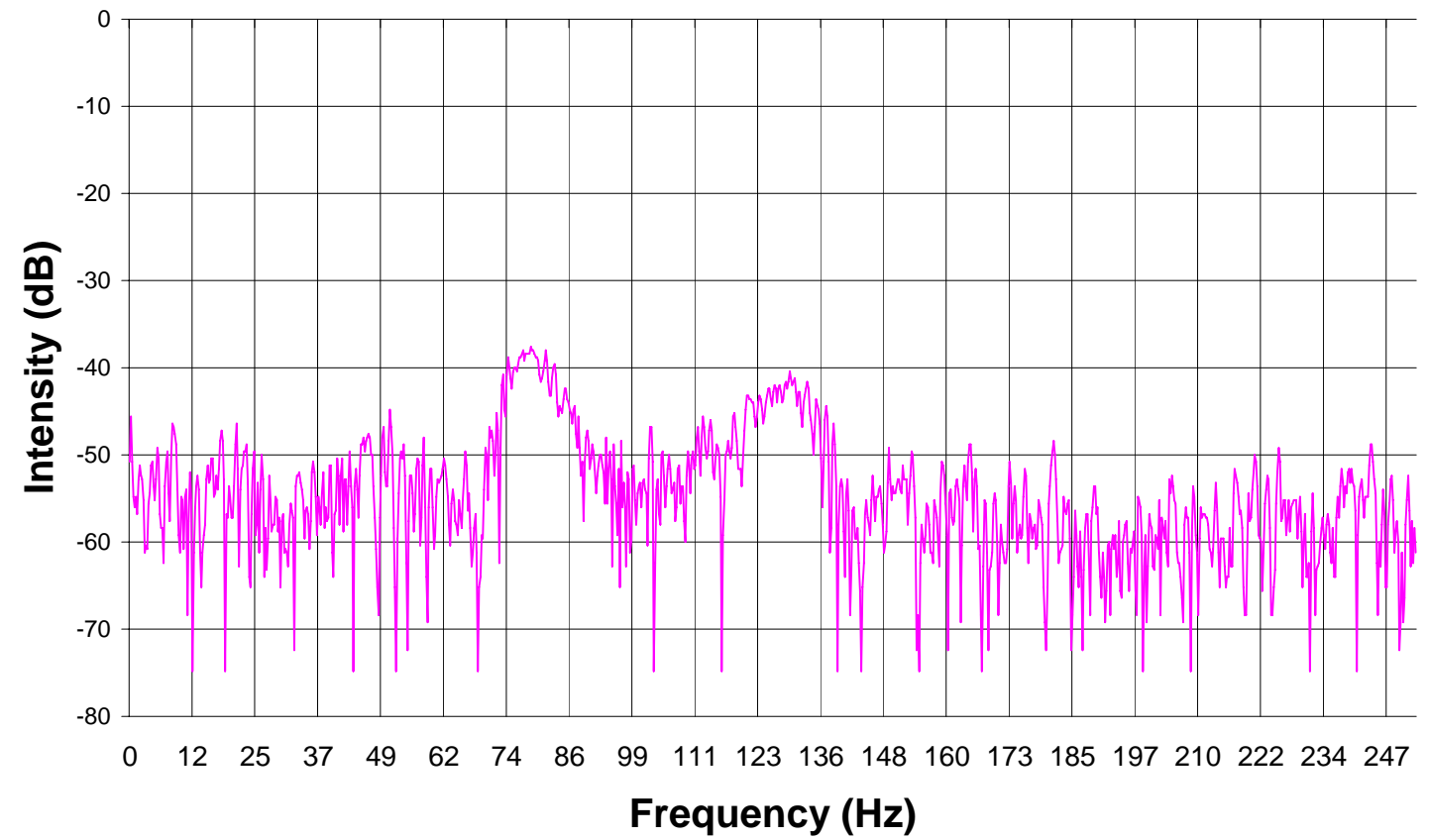

Figure 4.2 - Frequency response of the right vocal fold element

Time domain data can also be used to find out the resonance frequencies of the system. Figure 4.3 and Figure 4.4 show the response amplitude when the excitation frequency was changed around $131 \mathrm{~Hz}$. 


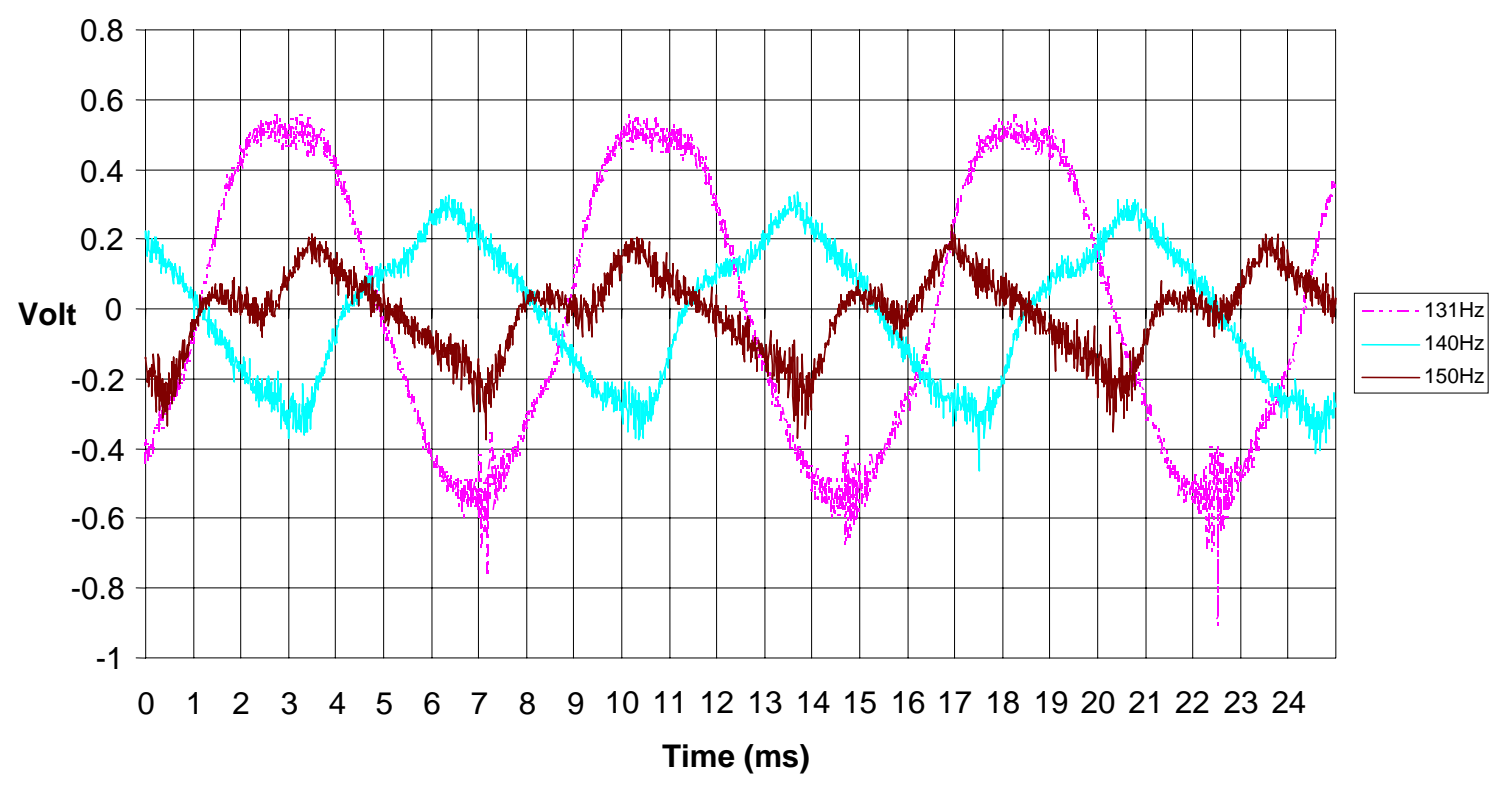

Figure 4.3 - Excited motion at frequencies 130, 140 and $150 \mathrm{~Hz}$ (left)

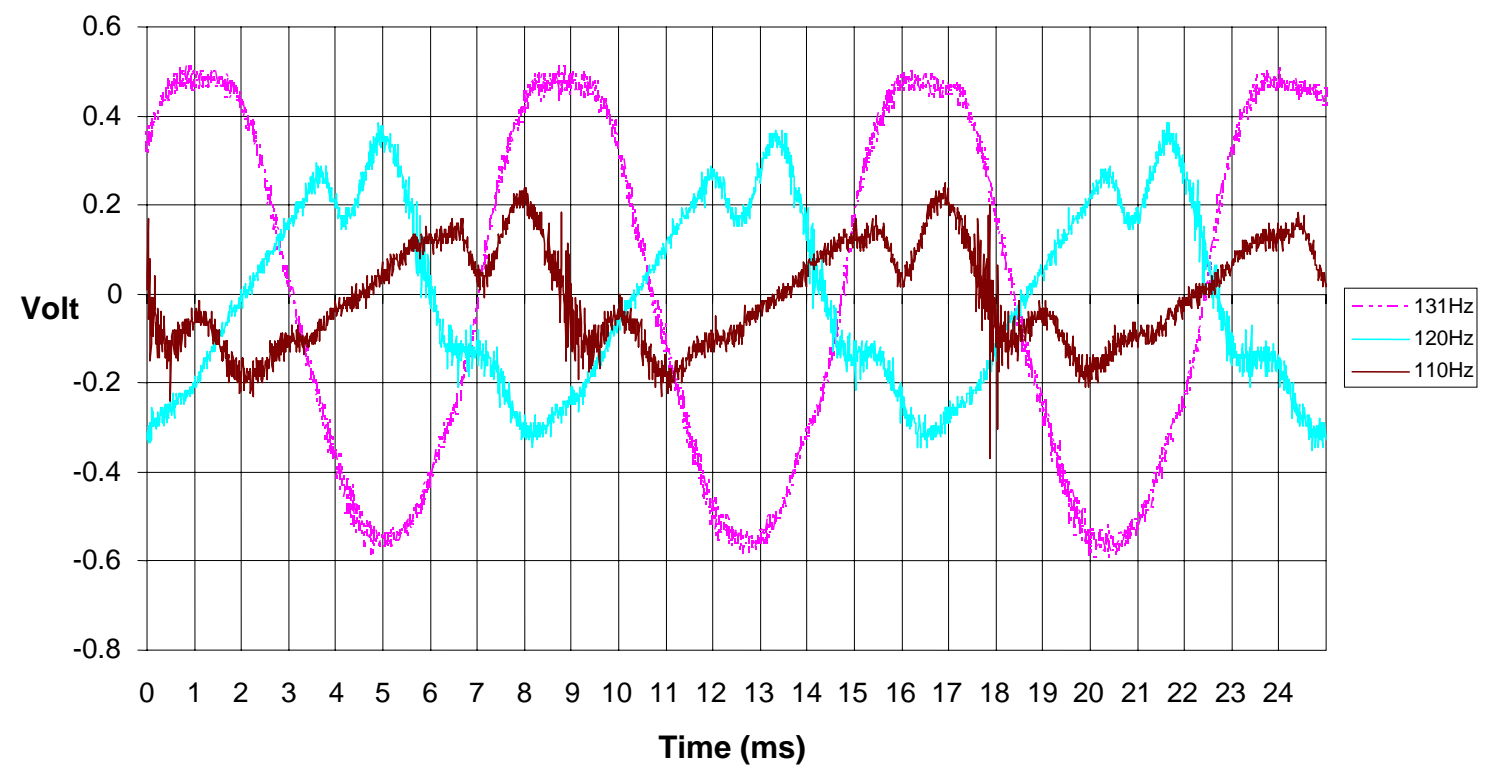

Figure 4.4 - Excited motion at frequencies of 110, 120 and $130 \mathrm{~Hz}$ (left) 


\subsubsection{Air Flow Results}

The following figures represent the velocity of the vocal fold midpoint during the model closure. Figure 4.5 shows the data from the left vocal fold element, and Figure 4.6 shows the data from the right element.

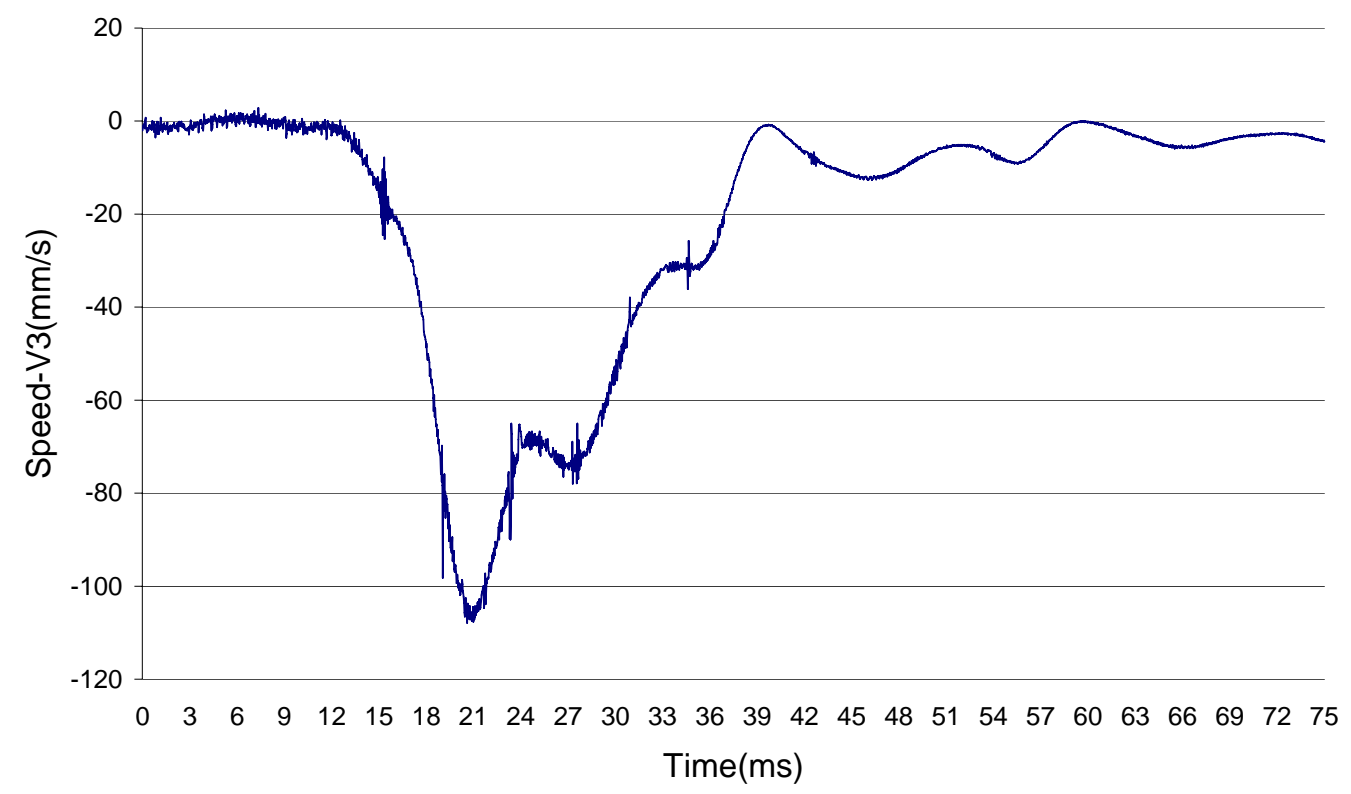

Figure 4.5 - Closure process of the left model

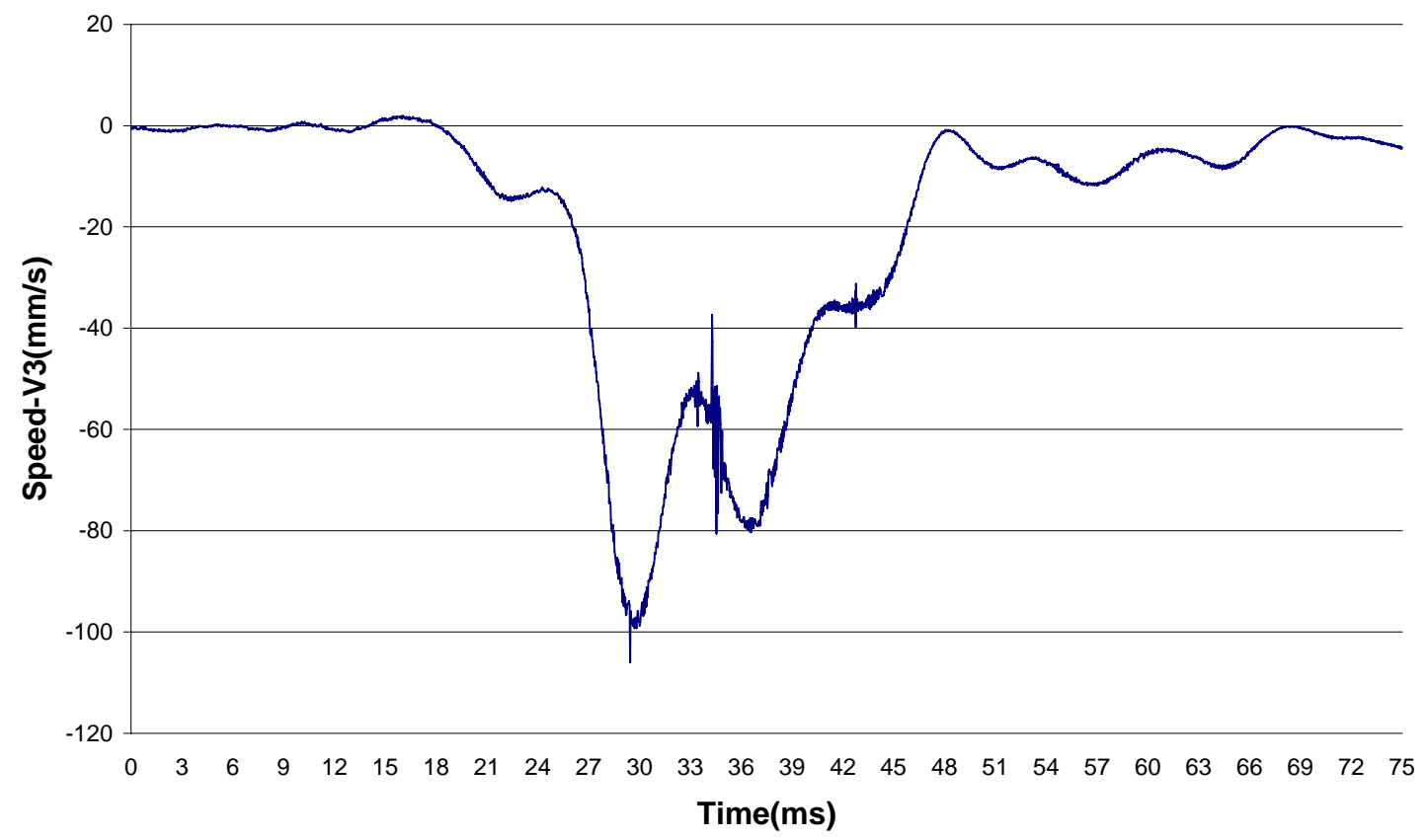

Figure 4.6 - Closure process of the right model 


\subsection{Finite Element Results}

The finite element results are determined using ABAQUS software. The whole model was divided into 276701 three-dimensional tetrahedral elements which consist of 57174 nodes. The total number of variables (degrees of freedom plus any Lagrange multiplier variables) in the model is 171522 . The mass of each of the model elements is $0.60749 \mathrm{~g}$.

\subsubsection{Vibration Results}

In ABAQUS, the frequency analysis utilizes the eigensolver to determine the eigenvalue and eigenfrunction. Implementing the geometrical dimensions, physical and mechanical properties results in eigenfrequency are shown in Figure 4.7. For various mode numbers, there are corresponding eigenfrequencies.

In most structural analysis, only the first few modes are of interest. Thus in this work only the first three eigenmodes and eigenfrequencies are considered. Figure 4.7 indicates that the first three natural frequencies for the realistic vocal folds are $125 \mathrm{~Hz}$, $184.88 \mathrm{~Hz}$ and $220.5 \mathrm{~Hz}$.

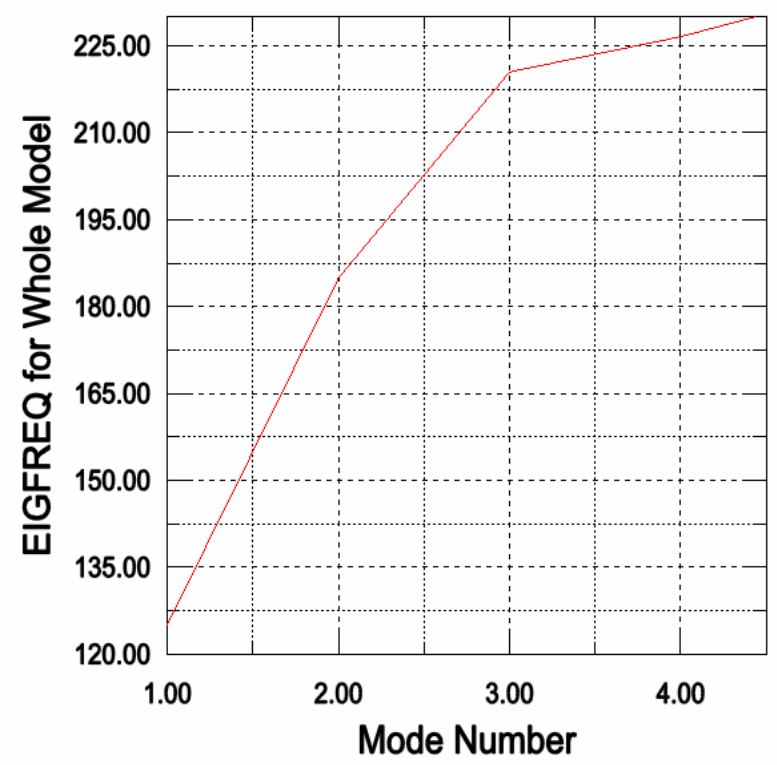

Figure 4.7 - Eigenfrequencies for different modes

Each eigenfrequency contrasts with an eigenmode. In previous study, Berry [19] found that the combination of the first two eigenmodes captures more than $95 \%$ of the variance of the vocal-fold vibration in normal phonation and more than $70 \%$ of irregular vocal-fold vibration. Thus, in this research, only the first two eigenmodes and 
eigenfrequencies will be discussed. Figure 4.8 shows the first two eigenmodes of the vocal fold. The first eigenmode at $125.07 \mathrm{~Hz}$ is the shape for the fundamental mode.
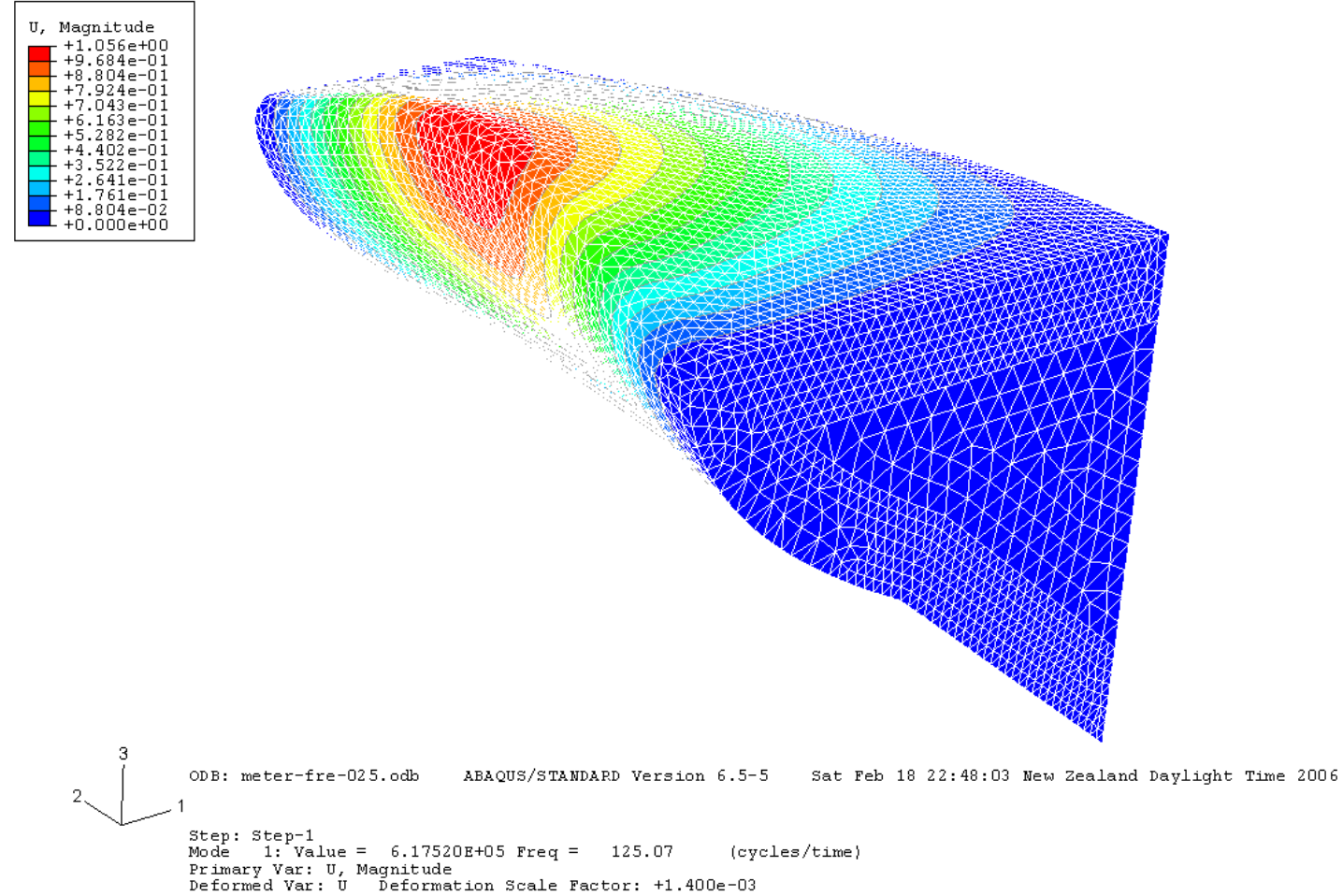

a
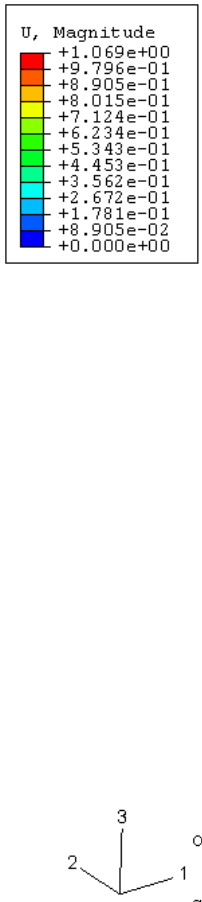

ODB: meter-fre-025. odb

ABAQUS/STANDARD Version $6.5-5$

Sat Feb 18 22:48:03 New Zealand Daylight Time 2006

Step:
Mode $2:$ Value $=1.34944 \mathrm{E}+06$ Freq $=184.88 \quad$ (cycles $/$ time)

Primary Var: U, Magnitude

b

Figure 4.8 - Vocal fold eigenmodes at (a) $125.07 \mathrm{~Hz}$, fundamental frequency (b) $184.88 \mathrm{~Hz}$, the first harmonic 


\subsubsection{Dynamic Response}

Glottal dynamics are an important output of the model. It consists of glottal closure and collision dynamics. The model behaviour during closure process is recorded and will be compared with clinical observation in the literature. The applied subglottal pressure are static with different amplitude $\left(P_{s}=600,800,1000,1200\right.$ and $\left.1500 \mathrm{~Pa}\right)$.

\subsubsection{Glottal Closure Dynamics}

Figure 4.9 shows the glottal closure process. The image of the up-left-side $(\mathrm{t}=0 \mathrm{~ms})$ represents the initial condition before the vocal folds closing. The up-right and downleft images represent the deformation of vocal folds at $1.0 \mathrm{~ms}$ and $1.8 \mathrm{~ms}$ after the vocal folds closure. The last image shows the full closure process taken about $2 \mathrm{~ms}$.

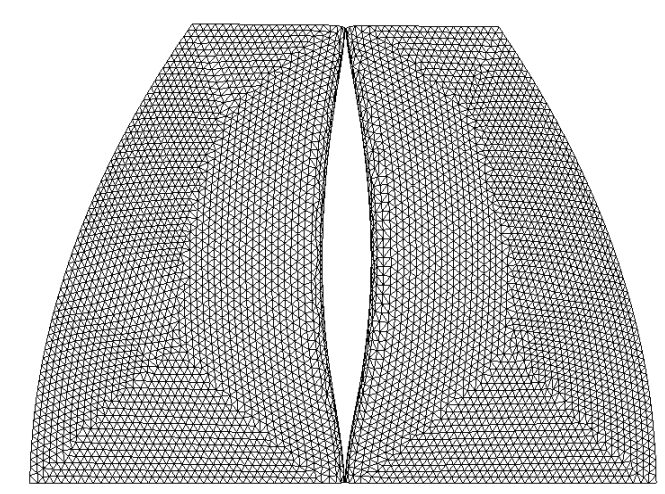

$\mathrm{t}=0 \mathrm{~ms}$

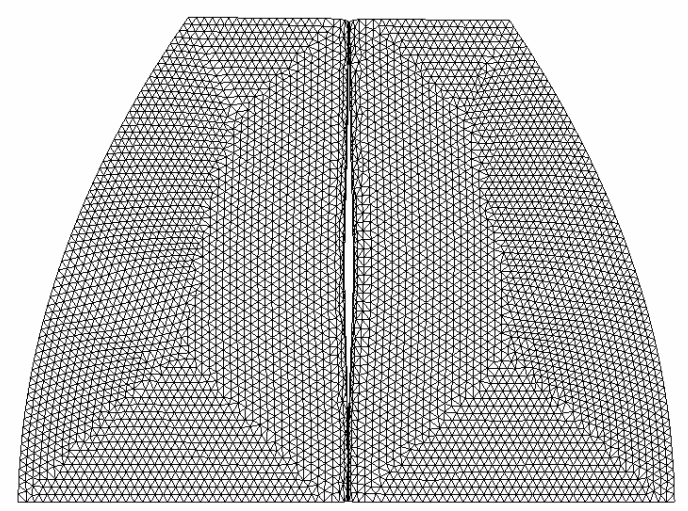

$\mathrm{t}=1.8 \mathrm{~ms}$

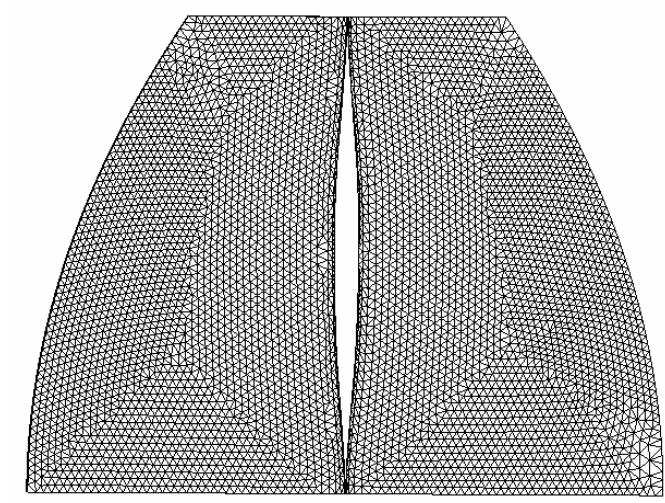

$\mathrm{t}=1.0 \mathrm{~ms}$

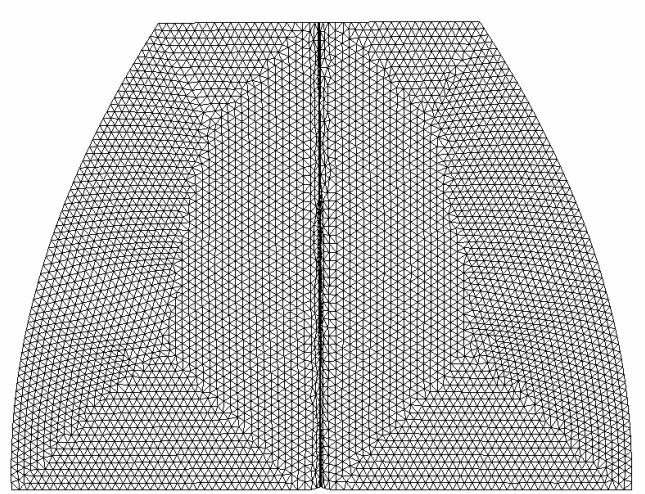

$\mathrm{t}=2.05 \mathrm{~ms}$

Figure 4.9 - The vocal folds model's closure process.

Five nodes were selected on the inferior portion of the right vocal fold edge as shown in Figure 4.10, which is the first portion of collision with the opposing fold. Node 249 is the midpoint of the edge, and the distance between the nearby nodes is $2.5 \mathrm{~mm}$. 


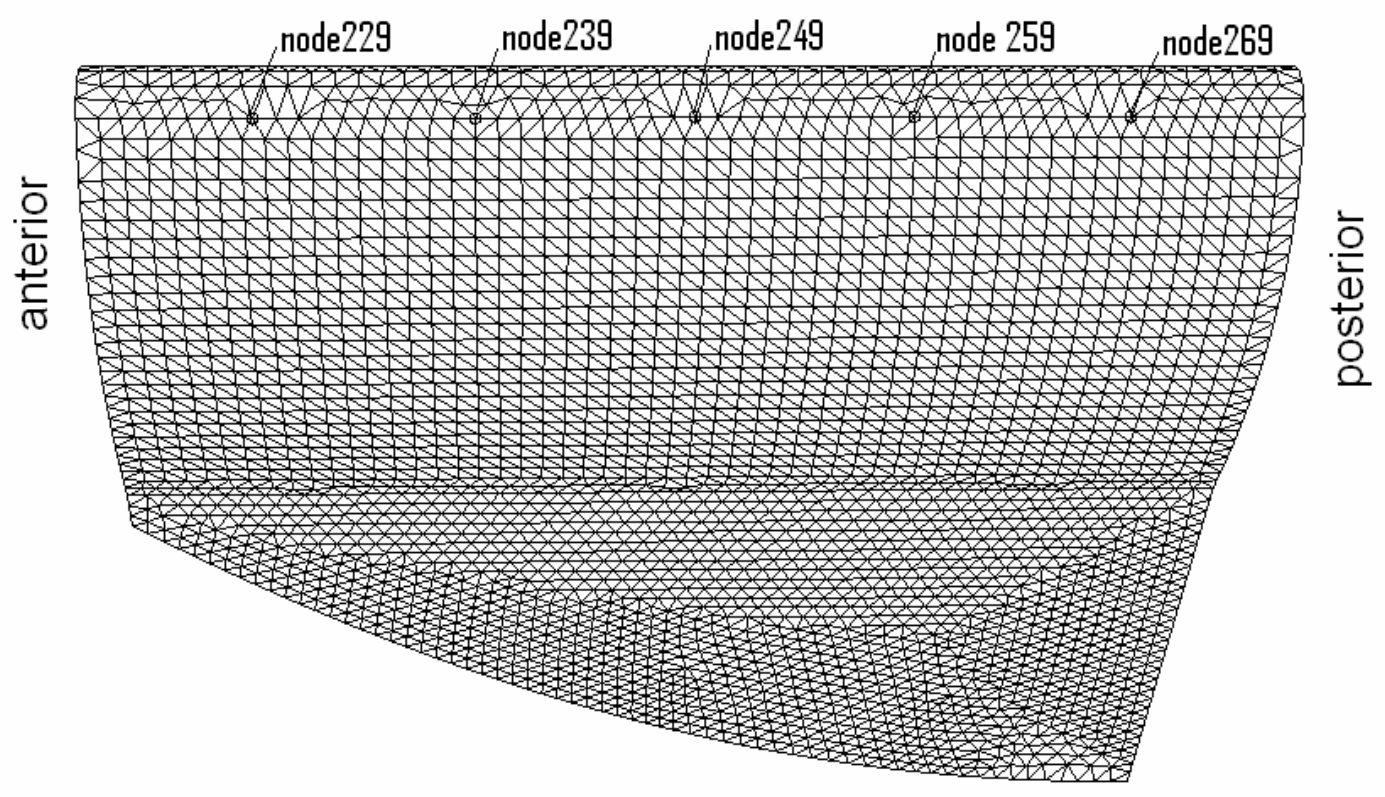

Figure 4.10 - Selected nodes for glottal opening test

Figure 4.11 shows that the movement of the selected nodes during closing process. Figure 4.12 shows the velocity of the selected nodes on the inferior edge. Both of the figures illustrate that, the portion near the midpoint of the inferior edge presents the greater displacement and collision velocity.

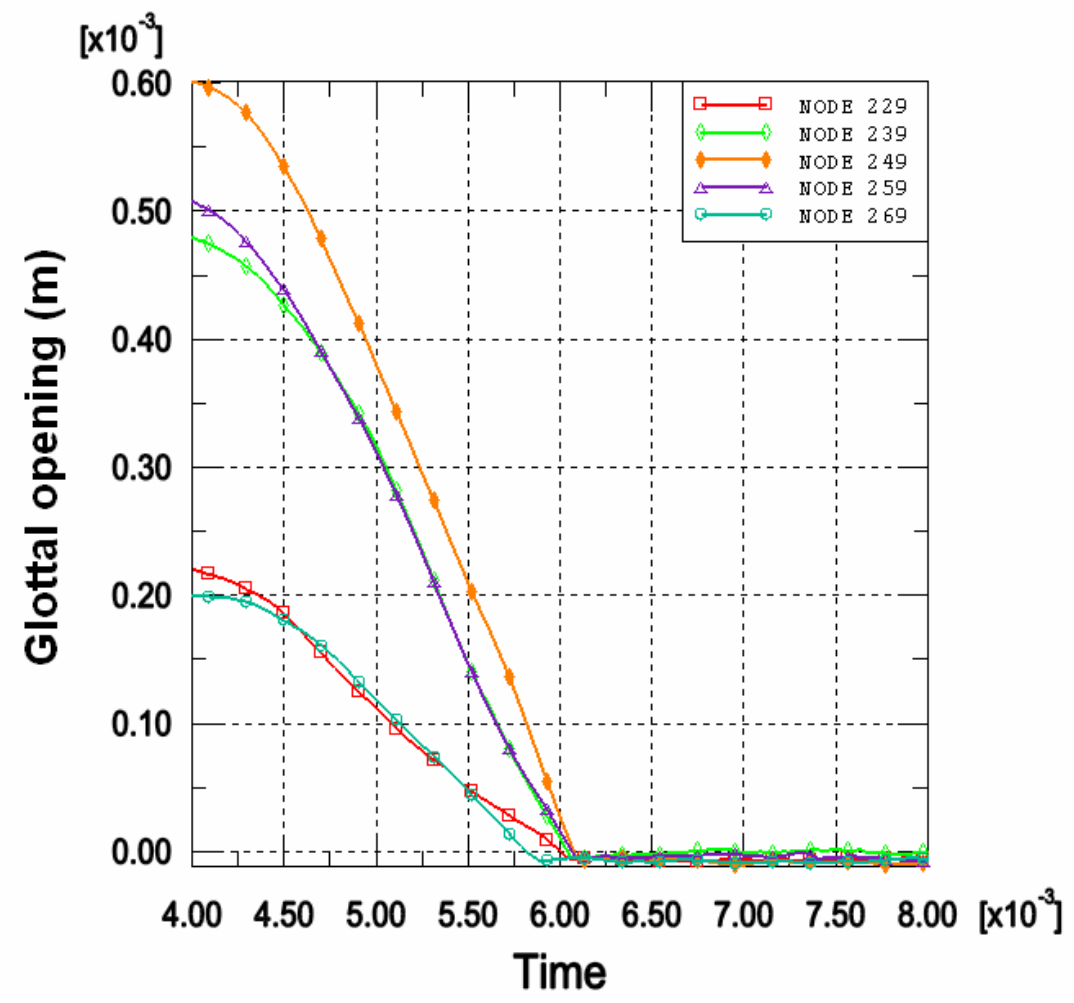

Figure 4.11- Closing time of different nodes $(\mathrm{Ps}=800 \mathrm{~Pa})$ 


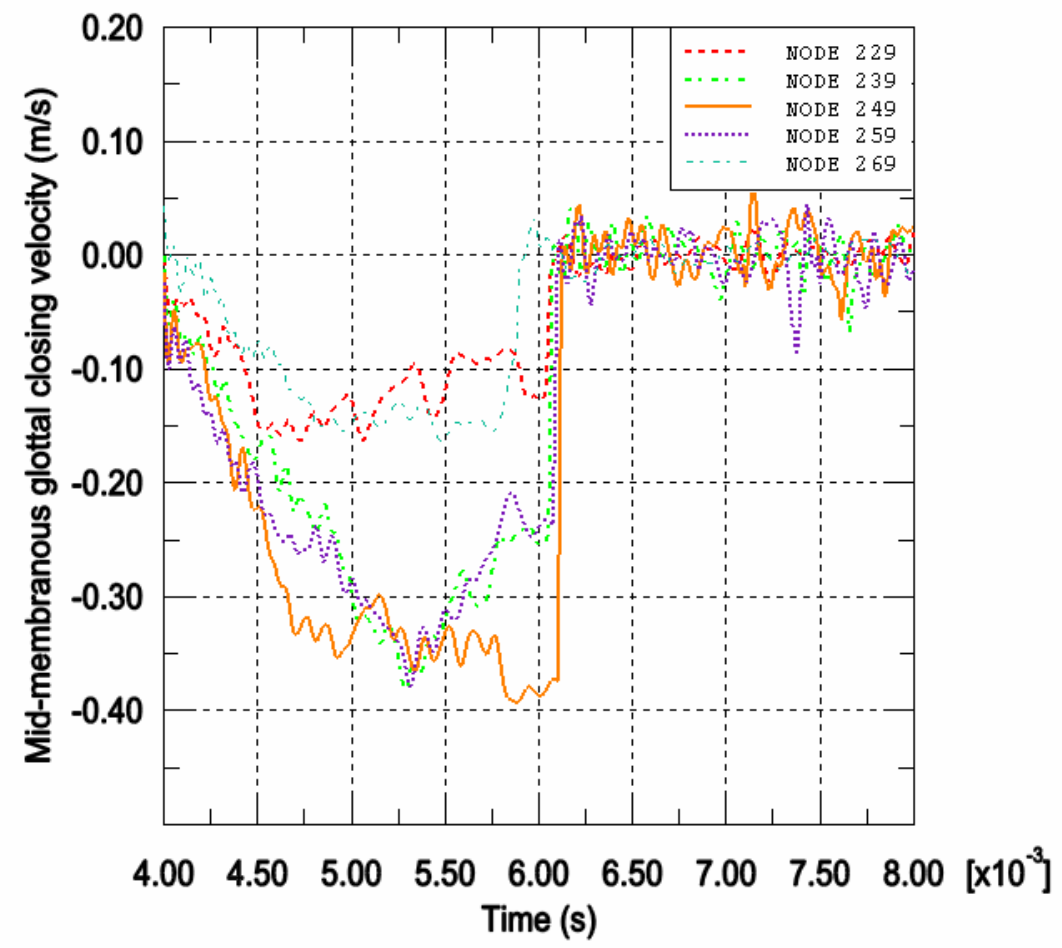

Figure 4.12 - Closing velocity of different nodes on inferior edge of the right vocal fold. $(\mathrm{Ps}=800 \mathrm{~Pa})$

From ABAQUS output, node $250\left(5.51^{-6}, 6.75^{-3}, 4.44^{-3}\right)$ is found to be the point of glottal opening amplitude and maximum impact velocity. It is just next to the midpoint $\left(5.51^{-6}, 7.00^{-3}, 4.44^{-3}\right)$ on the medial edge of the vocal fold. The model deformation varies with the subglottal pressure magnitude, which excited the model in the first step. Figure 4.13 shows the x position of node 250 during closure process. The arrow which points to the time of full closure and the curves shows the glottal opening amplitude. Furthermore, the relationship between the glottal opening amplitude and the subglottal pressure is shown in Figure 4.14. 


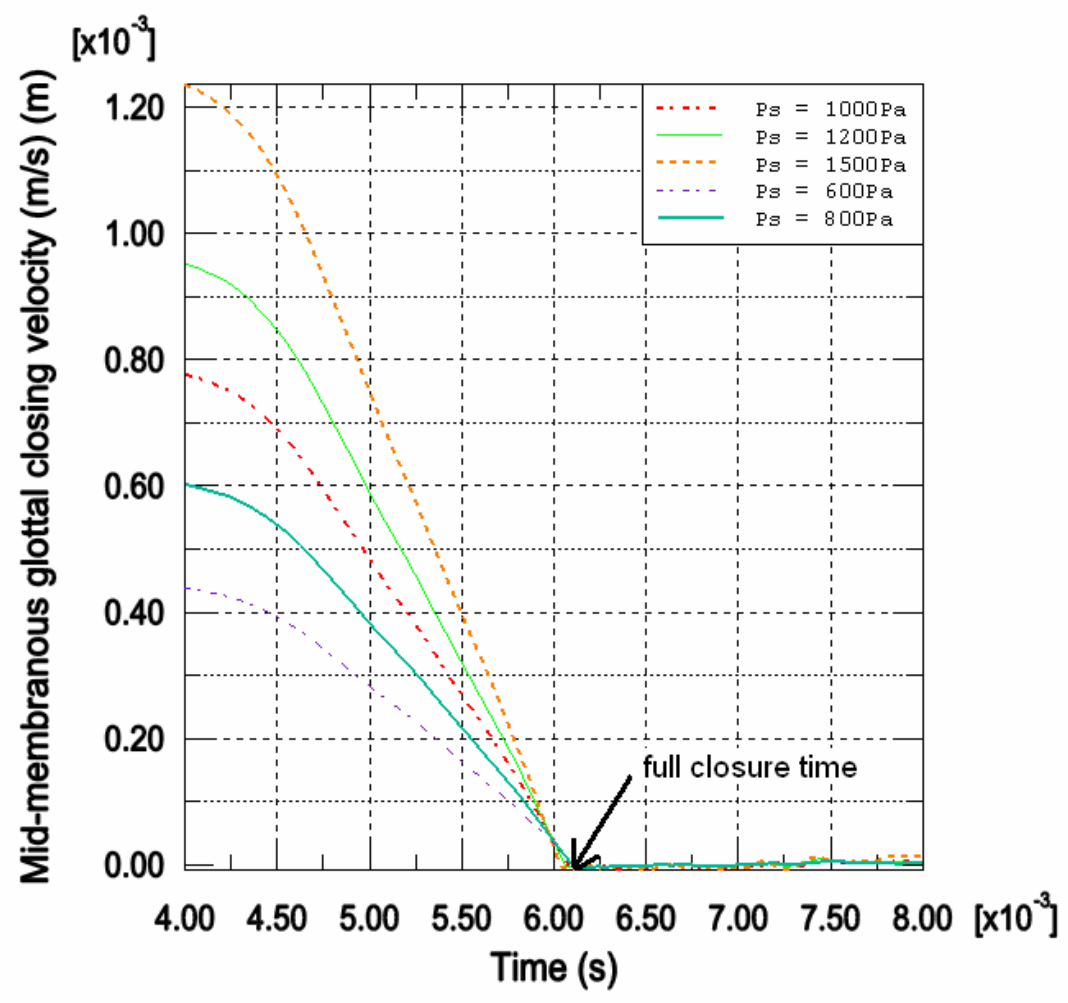

Figure 4.13 - Effect of the subglottal pressure on the vocal fold movement.

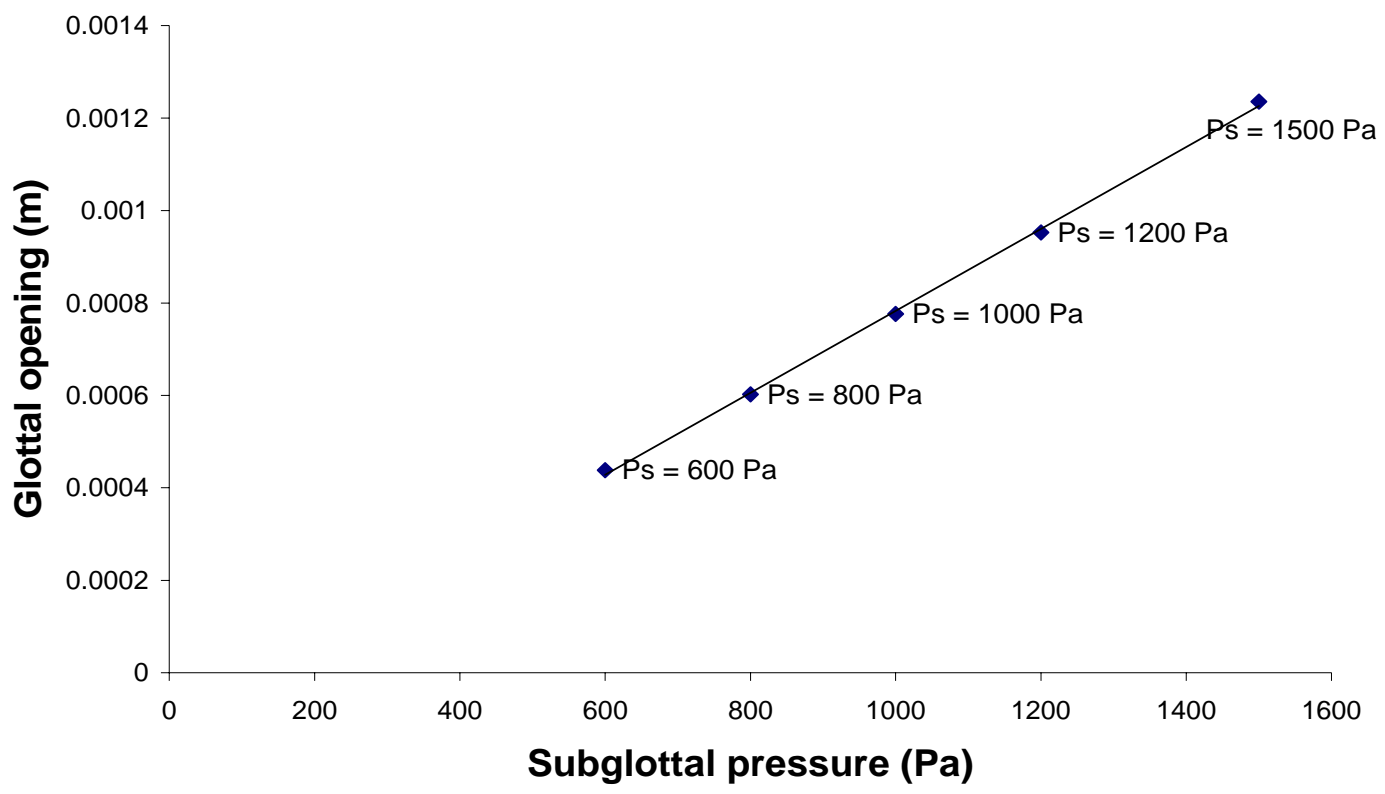

Figure 4.14- Glottal opening amplitude in $x$-direction

Figure 4.15 shows the velocities of node 250 in the $x$-direction excited by various subglottal pressures. During the collision, the velocity of the node changes to zero within $2.7^{-5}$ second and independent on the subglottal pressure. The impact velocity versus the subglottal pressure is approximately linear as shown in Figure 4.16. 


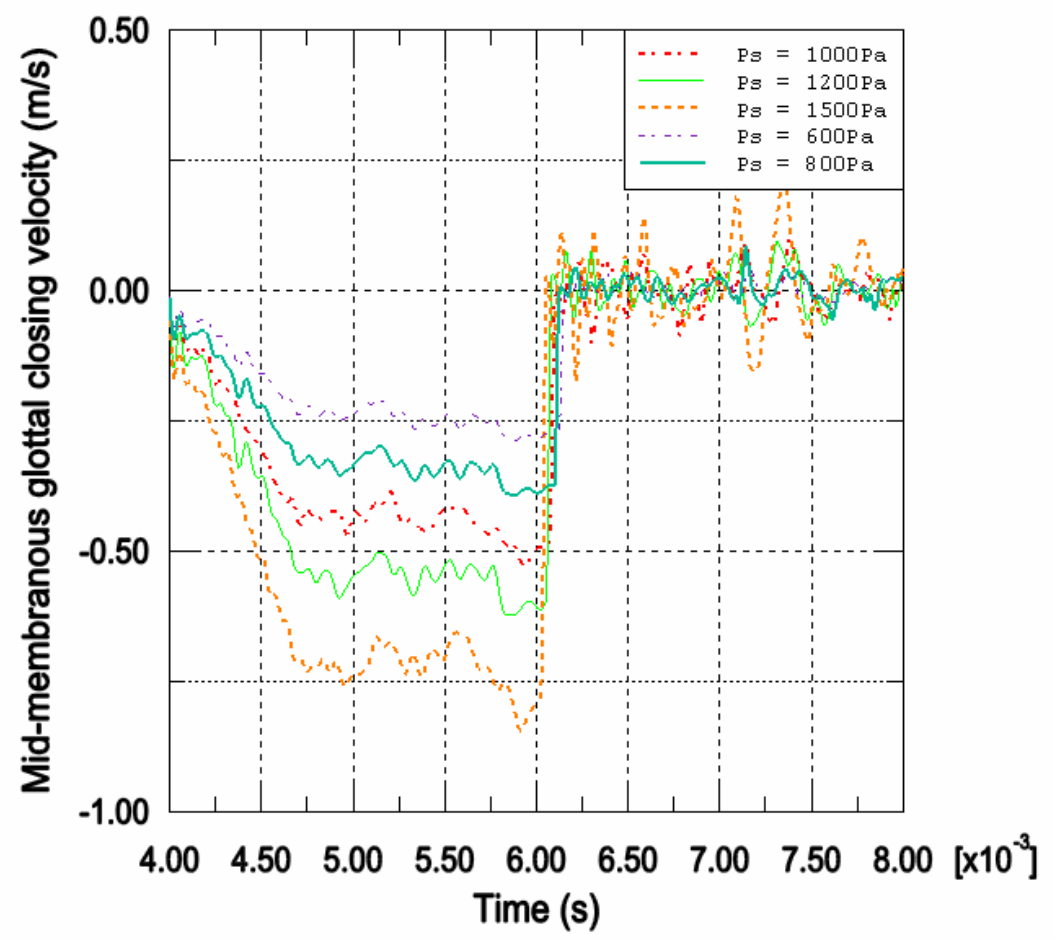

Figure 4.15 - Node 250 closing velocity under various subglottal pressures

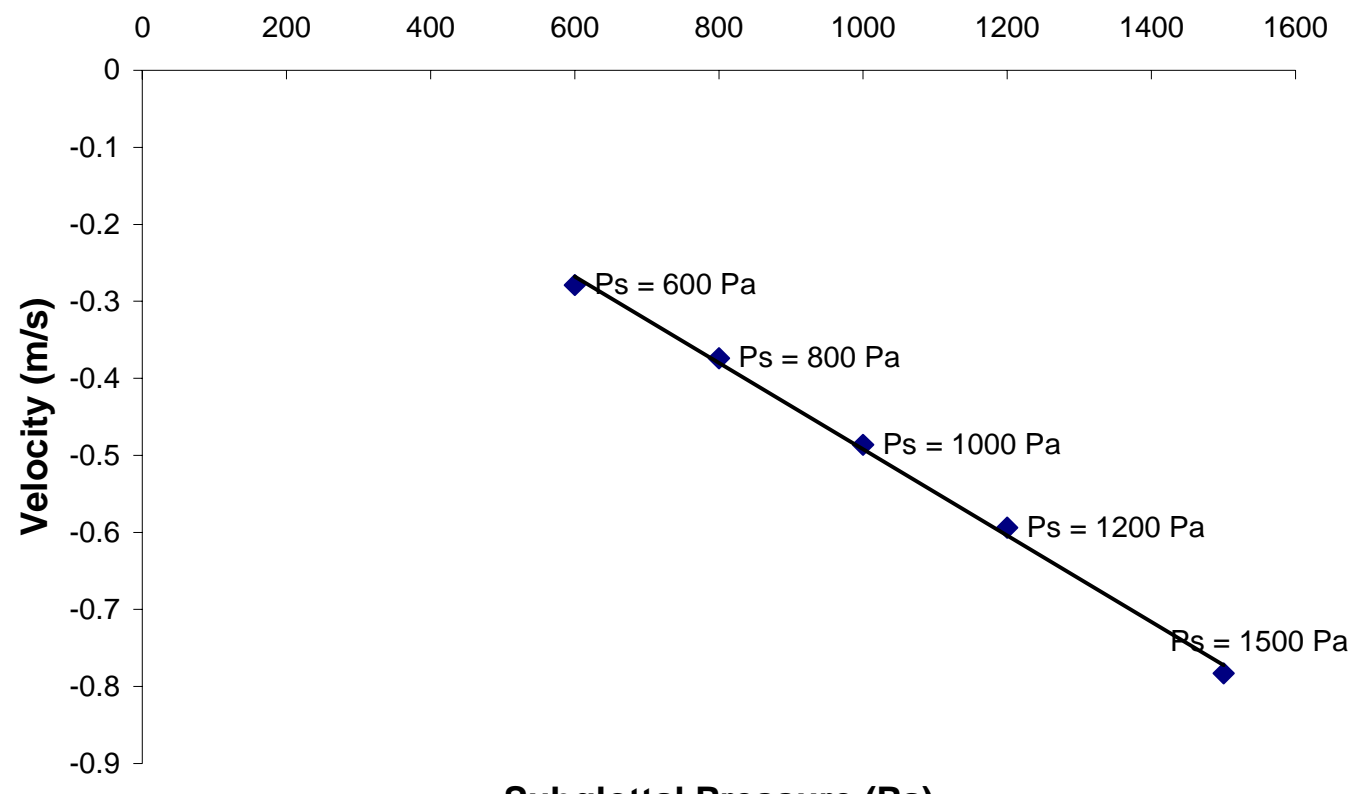

Subglottal Pressure $(\mathrm{Pa})$

Figure 4.16 - Closing velocity of node 250 in $x$-direction

\subsubsection{Glottal Collision Dynamics}

Normally, the contact force includes contact normal force (CNORMF) and frictional shear force (CSHEARF). Because the interaction between the folds was assumed 
frictionless, the frictional shear force between the vocal fold surfaces is zero. The contact force as shown in Figure 4.17 is the total force due to contact stress. The contact area is another important data in the glottal closure dynamics and is shown in Figure 4.18.

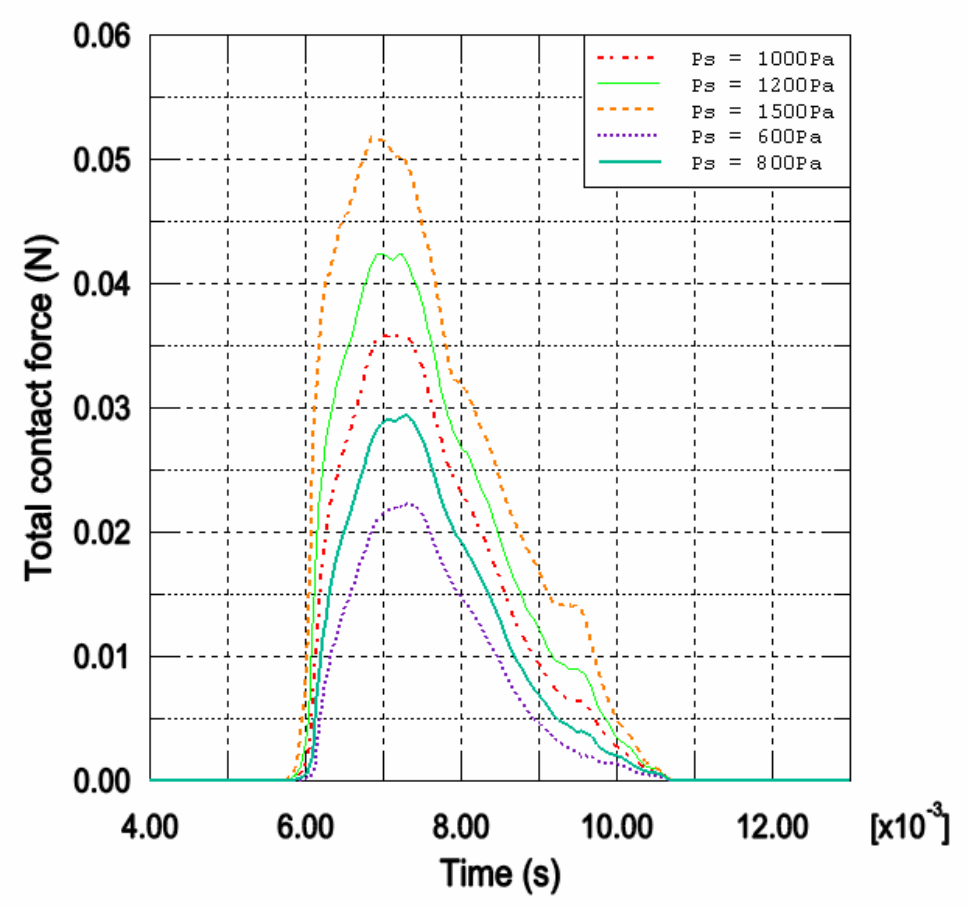

Figure 4.17 - Total contact normal force under various subglottal pressures (Ps=600, 800, 1000, 1200 and $1500 \mathrm{~Pa})$

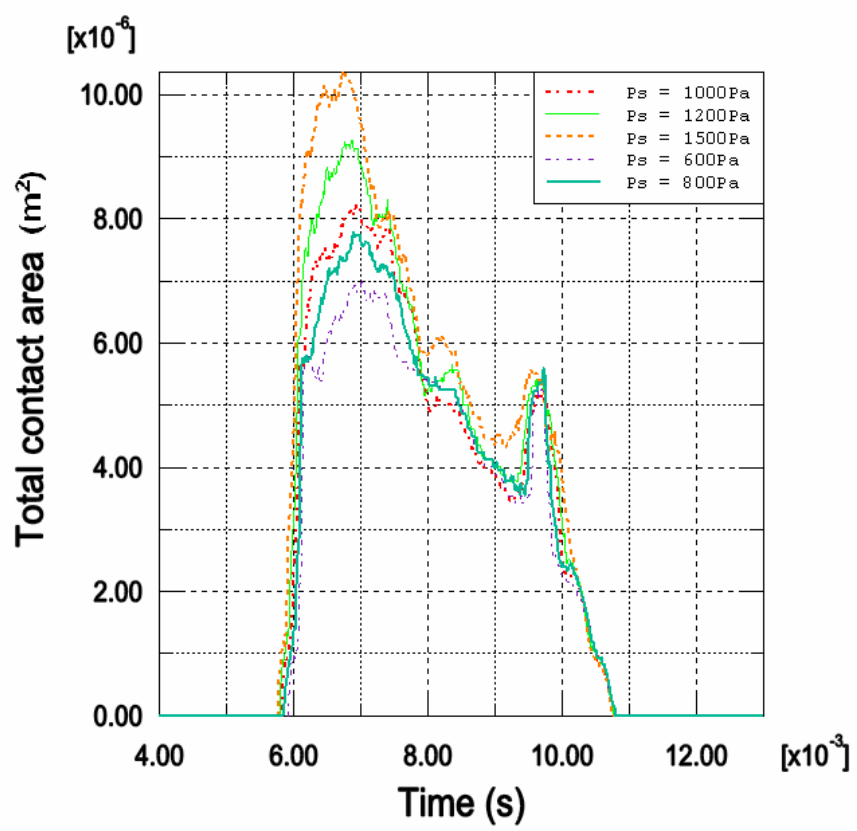

Figure 4.18 - Total contact area under various subglottal pressures $(P s=600,800,1000,1200$ and $1500 \mathrm{~Pa})$ 
As discussed in Chapter 2, the area around the midpoint of the vocal fold receives the maximum contact force and pressure [50]. From Figure 4.19, it is clear that, most of the points which receive the maximum contact normal were included in the selected area shown in Figure 2.14.

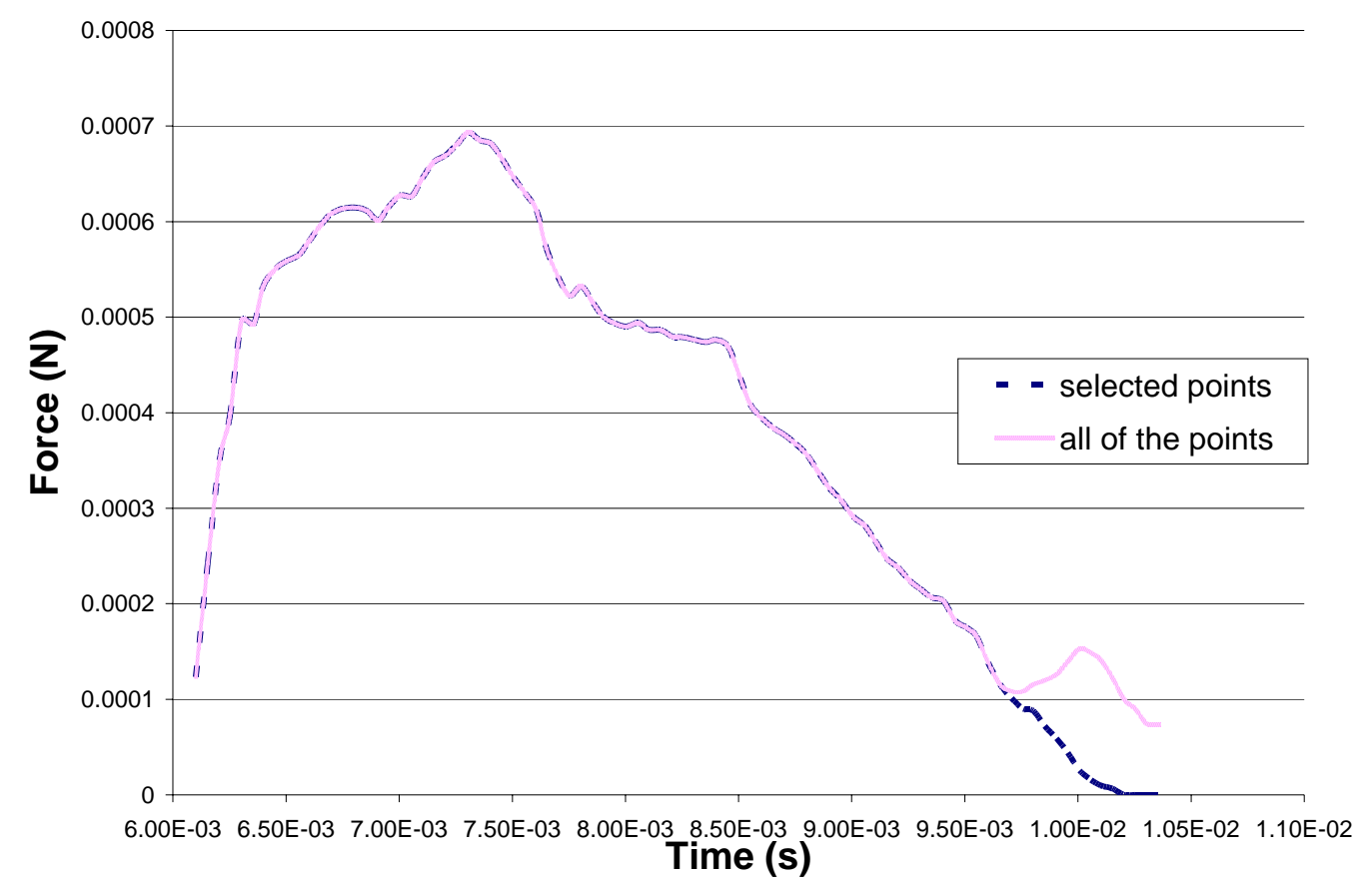

Figure 4.19 - Comparison of the max contact force between selected points and the whole model points (Ps $=800 \mathrm{~Pa})$

For general contact, CPRESS is calculated as the magnitude of the net contact normal force (the CNORMF vector) per unit area. If the contact stress is over $10 \mathrm{kPa}$, this may cause tissue damage [5]. From Figure 4.20, it is clearly indicated that some parts of the vocal folds are exposed to a contact stress over $10 \mathrm{kPa}$ during the vocal folds closure with an excited subglottal pressure of $800 \mathrm{~Pa}$. 


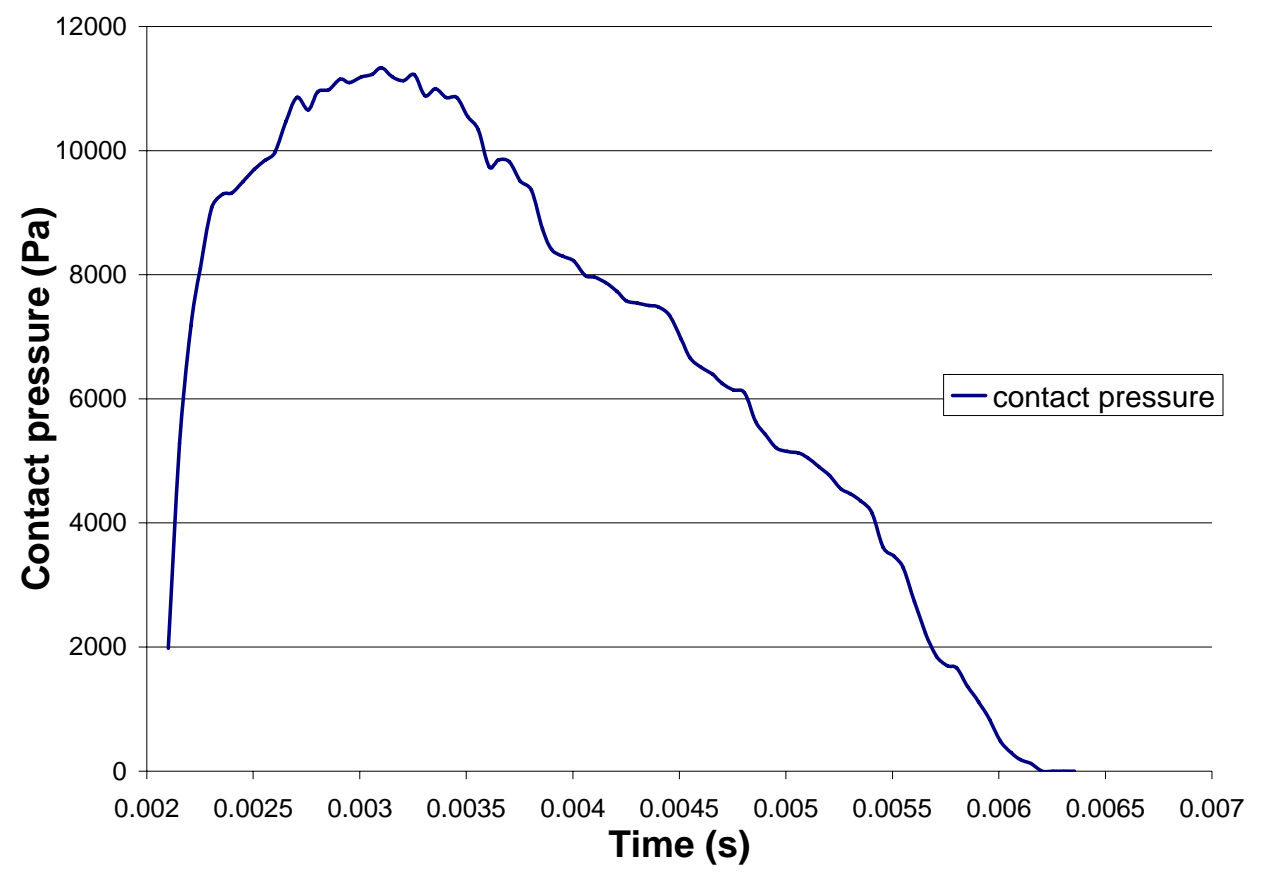

Figure 4.20 - Maximum contact stress of the selected points during closure $(\mathrm{Ps}=800 \mathrm{~Pa})$

However, there are still too many nodes in the selected area. The research need to concentrate on a smaller area with few nodes. From the resulting data, the following nodes, highlighted in Figure 4.21 were chosen. The elements around the selected nodes receive most of the maximum contact stress during the closure process.

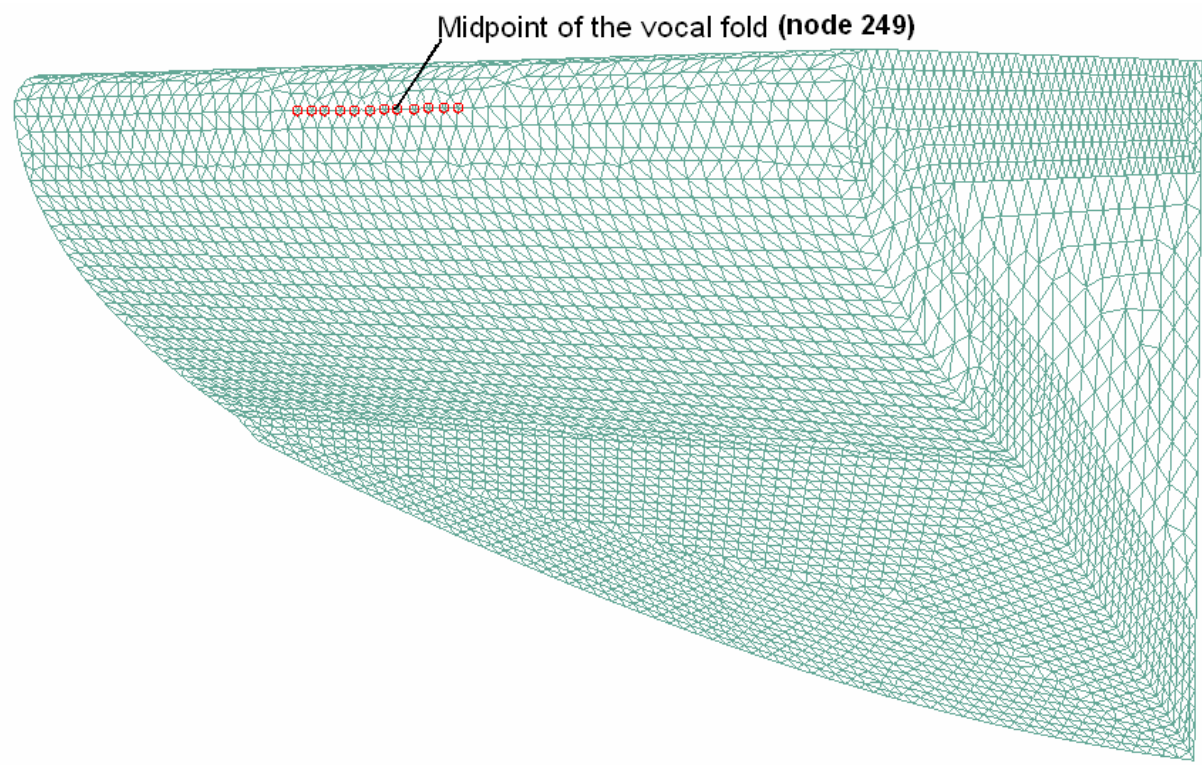

Figure 4.21 - The highlight points present the maximum contact normal force and contact stress during glottal pressure. 
The node number of the highlighted points from left to right is $242 \sim 253$ and their coordinates are from node $242\left(5.51^{-6}, 8.50^{-3}, 4.44^{-3}\right)$ to node $253\left(5.51^{-6}, 5.75^{-3}, 4.44^{-3}\right)$. Figure 4.22 shows the contact stress during closure when the subglottal pressure is $800 \mathrm{~Pa}$.

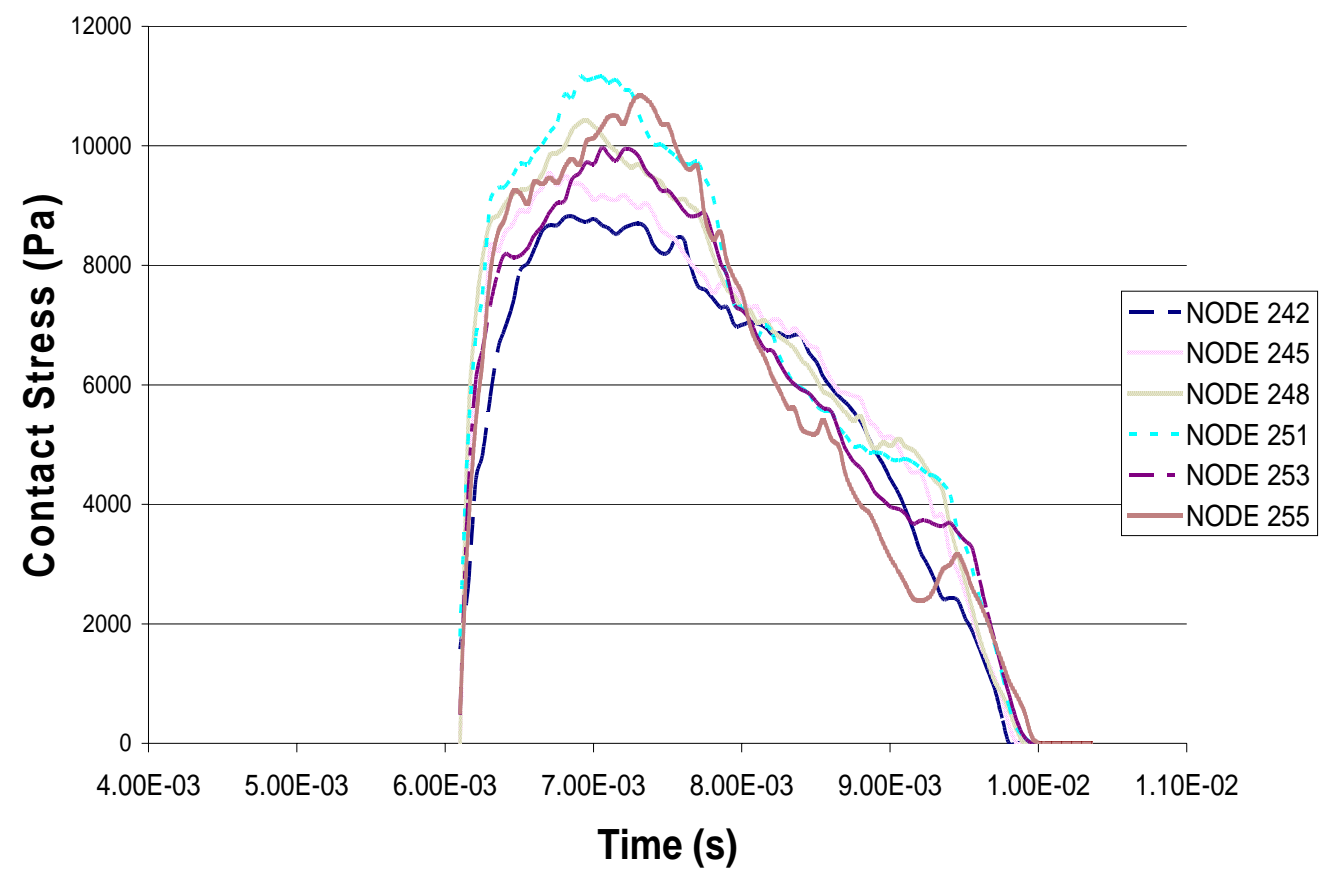

Figure 4.22 - Contact stress of the highlight points $(\mathrm{Ps}=800 \mathrm{~Pa})$ 


\section{Chapter 5 DISCUSSION}

\subsection{Introduction}

To the best of our knowledge, no previous work was found in the open literature on using layered structure with nonlinear material and geometry to study the frequency characteristics and dynamics response of the vocal folds. This chapter provides discussion on the frequency characteristics and dynamics response during closure of the experimental vocal folds tested in Chapter 2, the FEA models in Chapter 3 and comparison with some results from the literature.

\subsection{FEA Model Validation}

The general geometry and boundary conditions are validated by the experimental modal analysis results. The layered structure and viscoelastic material properties are validated by comparing with the in-vivo experimental results from published literature.

\subsubsection{Experiment Validation}

In this work, the experimental tests were generated to validate the theoretical results. The forced vibration experiment was based on the concept of vibrational resonance and modal analysis. The air flow excited experiment shows the dynamic characteristics under a step input.

\subsubsection{Forced Vibration Test}

As given in Chapter 3, the EMA (experimental modal analysis) model structure is slightly different from the real structure which the FEA model based on. This requires a new FEA model based on the same structure as EMA model. The main differences between the EMA model and the real structure are listed as follows:

1. The geometry size is twice larger than the real size. 
2. The whole EMA model is made of a silicone material instead of layered structure.

3. The properties of the silicone material are different from the real human tissue. They are obtained by the stress-strain test and given in Appendix III.

In the finite element analysis theory, a linear system can be decomposed into a set of independent vibration patterns which are called eigenmodes. Each of the eigenmodes corresponds to a natural frequency or eigenfrequency. Both the eigenmodes and the eigenfrequencies are characteristics of the system, and independent on the source of excitation. In real structures, every vibrating system possesses a unique resonance structure. The resonance frequencies and resonance modes can be seen as practical approximations of the eigenfrequencies and eigenmodes of the vocal folds [51].

The eigenfrequencies of the new FEA model were calculated using ABAQUS software as shown in Figure 5.1. The first two eigenfrequencies are: $81.87 \mathrm{~Hz}, 127.72 \mathrm{~Hz}$.

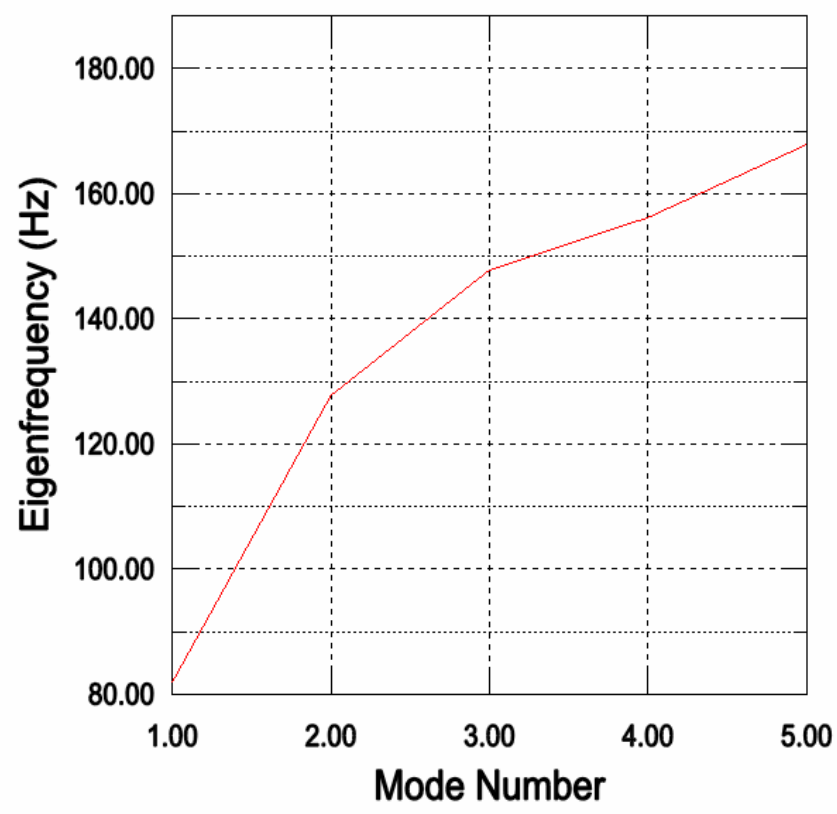

Figure 5.1 - Eigenfrequencies of different modes

Phasing resonance frequency is the simplest method to find out the resonance frequency in experimental modal analysis. That could be approached by both time domain data and frequency domain data. 
(1) Frequency domain results

For the EMA model, resonance frequencies obtained from the FFT results are shown in Figure 4.1 and Figure 4.2. The first two resonance frequencies are $75 \mathrm{~Hz}$ and $131 \mathrm{~Hz}$ for the left element and $79 \mathrm{~Hz}$ and $133 \mathrm{~Hz}$ for the right element. As can be seen there are three or more peaks observed within the experimental sweep range $10 \sim 200 \mathrm{~Hz}$ in the experiment. However, only two peaks were detected. This is attributes to the fact that the amplitude of excitation generates the shaker was too small to excite large frequencies. Furthermore, the two figures indicate that the maximum peak flatten due to the system's damping. Also the damping attribute to the elimination of higher frequency. Thus higher resonance frequencies can't be detected.

(2) Time domain results

Figure 4.3 and Figure 4.4 show that when any excitation frequency changes around $131 \mathrm{~Hz}$, the response of the system decreases. The definition of resonance frequency is that at resonance frequency, any change, no matter how small, in the frequency of excitation causes a decrease in the response of the system [44]. Then this is confirmed that $131 \mathrm{~Hz}$ is one of the resonance frequencies of the left vocal fold model.

Table 5-1 Comparison of theoretical results and experimental results

\begin{tabular}{|c|c|c|c|c|}
\hline \multicolumn{5}{|c|}{ The natural frequencies } \\
\hline & ABAQUS & \multicolumn{2}{|c|}{ FFT results (Hz) } & \multirow{2}{*}{$\begin{array}{c}\text { Time domain } \\
\text { results }(\mathrm{Hz})\end{array}$} \\
\cline { 3 - 4 } & $(\mathrm{Hz})$ & left & right & Not detected* \\
\hline First & 81.87 & 75 & 79 & 131 \\
\hline Second & 127.72 & 131 & 133 & 13 \\
\hline
\end{tabular}

* the reason will be discuss in section 5.3

\subsubsection{Air Flow Test}

To simulate a step input, a constant pressure air flow was applied to the vocal folds and then suddenly removed. At this stage, the folds moved freely with a bit of oscillation. The results of this test are presented in Figure 4.5 and Figure 4.6, for the left and right element of the model respectively. The two figures indicate that there is no positive 
velocity (opening direction) during the closure process of the experimental model. That means the vocal folds will not come back to its original position. Evidently, this does not agree with the clinical observation or the ABAQUS simulation results. However, this discrepancy is attributed to the inaccuracy in the experimental setup. The laser sensor is a non-contact sensing device which measures the movement of a point along the laser ray; i.e. it traces the movement along a straight line. In the present case when the vocal folds bent, different points were detected by the same laser. This is illustrated in Figure 5.2. Unfortunately there was no displacement tracking device available when this experiment was undertaken.

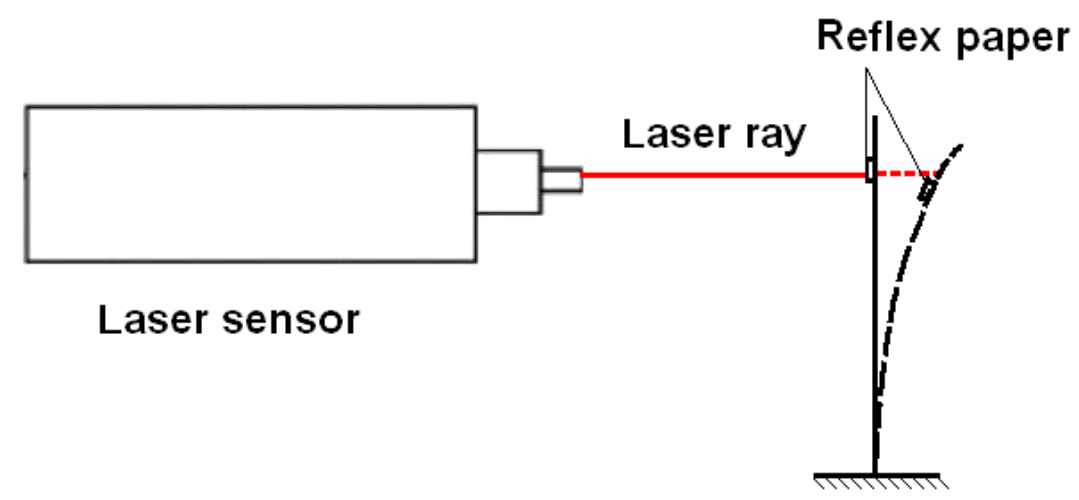

Figure 5.2 - The detected point will change if the object bends.

Figure 5.3 shows the closure process of the right element of the model. It is clearly indicated that the frequency of out-of-plane self-oscillation is between $130-140 \mathrm{~Hz}$. This is in agreement with the first experiment which showed that one resonance frequency is $131 \mathrm{~Hz}$ and the theoretical value. A more accurate result of this work should have been done using a high speed movie camera which was not available at the time of experiment. 


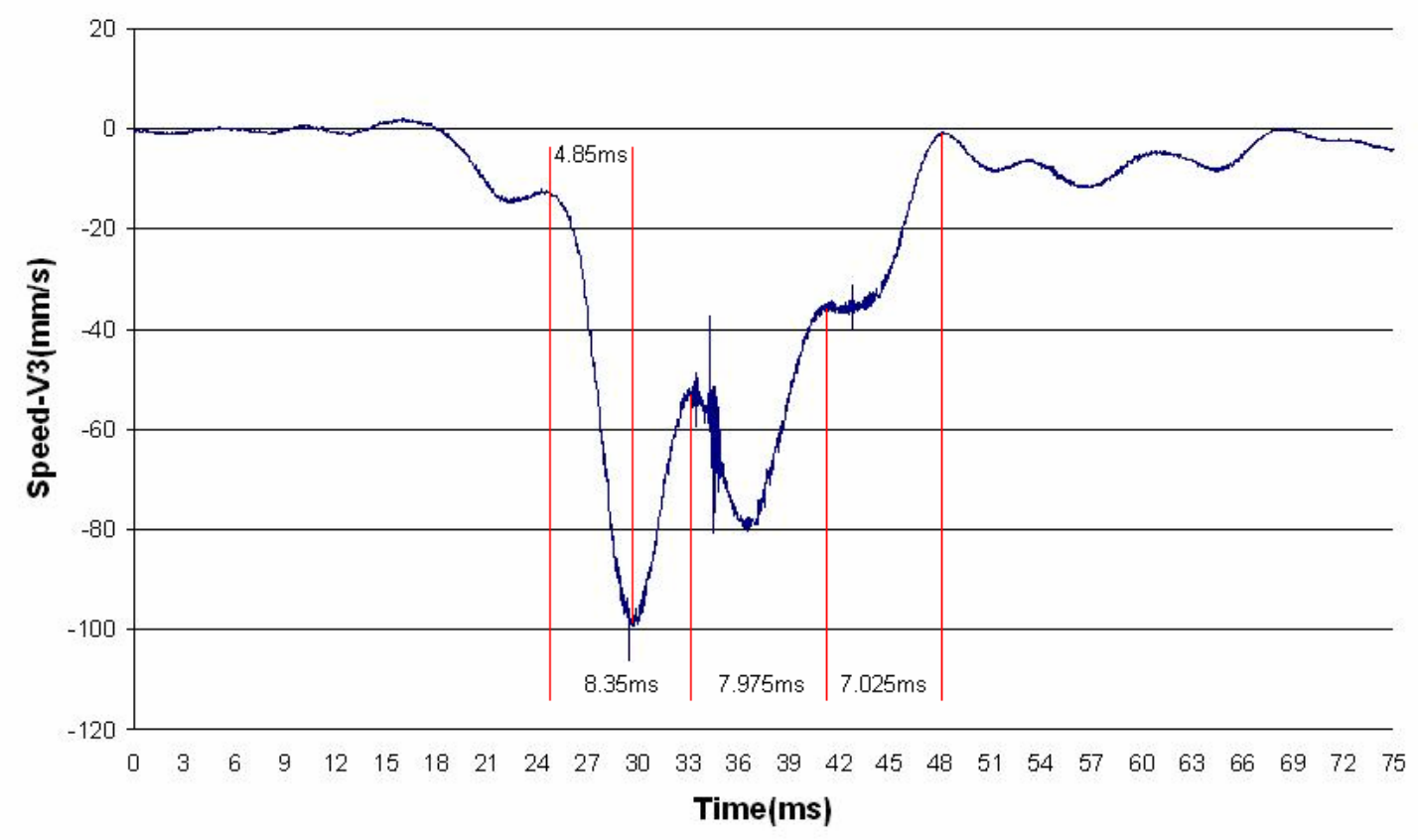

Figure 5.3 - Closure process of the right model

As shown in Table 5-1, the experimental results of the resonance frequencies and the theoretical results of eigenfrequencies agree quite well. From the experiment, it is believed that the ABAQUS model does represent the complex model with nonlinear geometry and viscoelastic properties. Although the resonance modes of the system could not be observed in the experiment, the frequency agreement is strong enough to validate the FEA model geometry, boundary conditions and mesh method.

\subsubsection{Comparison with Literature}

Experimental resonance studies of the vocal folds have been performed on excised larynxes, and on in vivo human subjects [52-54]. Summarizing the results on excised larynxes, Kaneko [54] indicated that two prominent resonances appeared in the excised larynxes, with no tension applied. The frequencies of the first two resonances were 120 and $180 \mathrm{~Hz}$, respectively. In 2000, Svec [52] duplicated Kaneko's experiment utilizing new equipment such as an endoscope. This study detected three resonances in the folds at 110,170 and $240 \mathrm{~Hz}$.

In the present FEA model designed in Chapter 2, the first three eigenfrequencies are 125 $\mathrm{Hz}, 184.88 \mathrm{~Hz}$ and $220.5 \mathrm{~Hz}$. Table 5-2 shows that these frequencies are in excellent agreement with the in-vivo experimental testing. The agreement corroborate the layered structure and material properties assigned in the FEA model. Thus the FEA model 
developed in Chapter 2 is a dependable tool which could be used for future development and investigation.

Table 5-2 Comparison of theoretical results and in-vivo experimental results

\begin{tabular}{|c|c|c|c|}
\hline \multirow{2}{*}{} & Natural frequencies \\
\hline \multirow{2}{*}{ ABAQUS results (Hz) } & \multicolumn{2}{|c|}{ In-vivo Experimental results (Hz) } \\
\cline { 2 - 4 } & & Kaneko & Svec \\
\hline First & 125 & 120 & 110 \\
\hline Second & 184.88 & 180 & 170 \\
\hline Third & 220.5 & Not available & 240 \\
\hline
\end{tabular}

\subsection{Frequency Analysis}

As mentioned before, the combination of the first two eigenmodes captures more than $95 \%$ of the variance of the vocal-fold vibration in normal phonation and more than $70 \%$ of irregular vocal-fold vibration [19].
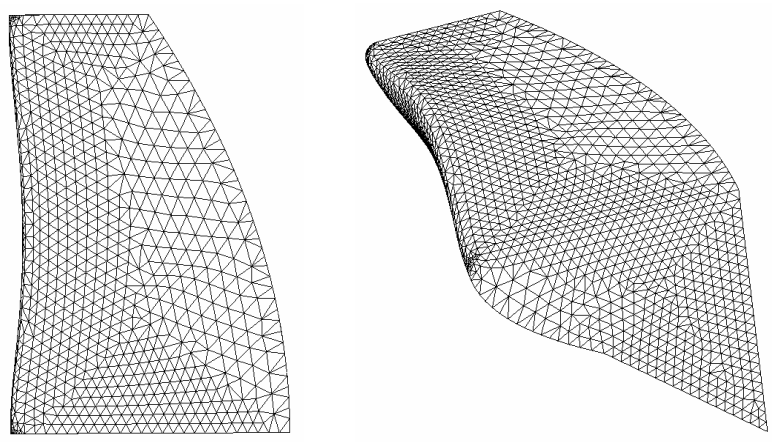

a
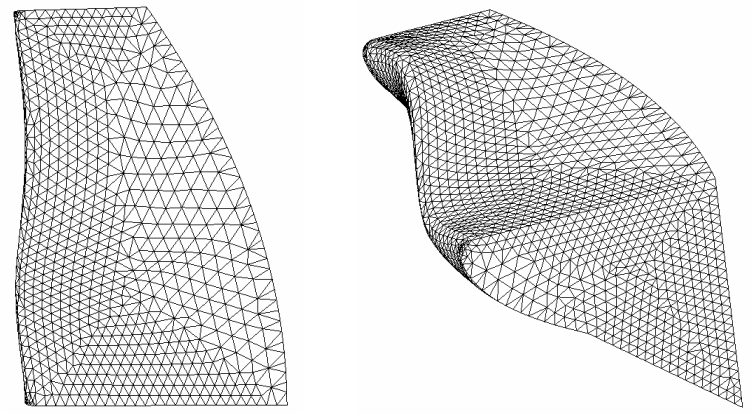

b

Figure 5.4 Eigenmodes (a) one (at $81.87 \mathrm{~Hz}$ ) (b) two (at $127.72 \mathrm{~Hz}$ ) 
Therefore, it is worth to focus on the first two resonance frequencies, $75 \mathrm{~Hz}$ and $131 \mathrm{~Hz}$. Figure 5.4 shows the first and second eigenmodes of the EMA model.

The first one is associated with in-plane (medial-lateral) oscillation. Resonance at this frequency will cause the two elements (right and left) of the model open and close. This mode is responsible for abduction and adduction of the folds in a vibration cycle. The second frequency, $131 \mathrm{~Hz}$, is associated with both in-plane (medial-lateral) and out-ofplane (superior-inferior) vibration of the folds. That is to say, at this resonance, the folds will vibrate in the transverse direction. This plays an important role in glottal shaping which presents the up-down motion of the vocal folds. However, the laser sensor used in the experimental investigation can only detect the out-of-plane vibration (superiorinferior) and then detect the vibration generating at $131 \mathrm{~Hz}$ rather than at the $75 \mathrm{~Hz}$. The frequency characteristic gives the reason why it was hard to detect the resonant vibration at the first resonance frequency using one-dimensional detection equipment.

The eigenmodes of the FEA model based on the real structure are shown in Figure 4.8. It is clear that they are similar as the eigenmodes of the EMA model discussed above. Therefore, in the FEA model, the first natural frequency $125.07 \mathrm{~Hz}$ is associated with in-plane vibration and the second natural frequency $184.88 \mathrm{~Hz}$ is associated with out-ofplane vibration.

During phonation, when the subglottal airflow is periodic input around the fundamental frequency $125 \mathrm{~Hz}$, the responses of vocal folds vibration will increase dramatically. The impact stress between the vocal folds will be much larger than that under a step input. When the glottal airflow is at the second natural frequency, the main movement of the vocal folds vibration will be out-of-plane. The main force between the vocal folds will be the shear force. If the vocal folds are in healthy situation, the friction ratio between the vocal folds will be very small and shear force could be ignored. If the vocal folds keep work for a long time, and the mucus doesn't work very well, the shear force will increase and damage the vocal folds tissue. There is no experimental data about the friction ratio between the vocal folds and what shear stress magnitudes cause tissue failure. Therefore, it is impossible to comment on which stress is the main risk of tissue damage in this situation. 


\subsection{Dynamic analysis}

The glottal dynamics is separated into two parts: closure dynamics and collision dynamics.

\subsubsection{Glottal Closure Dynamics}

From Figure 4.9, it is shown that the glottal closure starts from the anterior and posterior ends, and proceeds towards the middle region. For more details, as shown in Figure 4.11, the node 269 near the posterior end achieves the mid-plane first, and then the node 229 near the anterior end reaches the mid-plane and finally the midpoint node 249. Because the two vocal folds are designed symmetric in this model, the movement of the vocal folds are symmetric too. Thus, once the inferior surfaces arrive to the mid-plane, they will contact with each other.

As demonstrated in Chapter 4, node 250 is the point of maximum glottal opening and maximum impact velocity and it is the last point contacting with the opposing fold. Therefore, in this discussion, we will focus on the movement of node 250 rather than the whole vocal fold.

In previous observations of in vivo testing of fully abduction and adduction [55], the glottal opening amplitude responds positively to the magnitude of the subglottal pressure. That is to say as the pressure increase, the opening amplitude increase too. Figure 4.13 and Figure 4.14 show that the displacement in $x$-direction is also positive and approximately follow a linear trend with the subglottal pressure.

The full glottal closure time is another important characteristic of this investigation. This time is from the initial closure condition $(\mathrm{t}=4 \mathrm{~ms}$ in this model) to full closure which is defined as zero glottal area. Figure 4.13 shows that the full glottal closure time is constant of about $2.05 \mathrm{~ms}$ under various subglottal pressures. In conclusion, it is independent of the subglottal pressure. Figure 4.13 and Figure 4.15 show the displacement and velocity behaviour of node 250 during closure process in $x$-direction. This process may be divided into two steps:

(1) The first step takes about $0.72 \mathrm{~ms}$ from the initial condition. The acceleration is approximately constant in this step. 
(2) The second step is from the end of the first step to fully glottal closure. This step takes about $1.33 \mathrm{~ms}$. In this step, the velocity is constant until the contact of opposite fold. The impact velocity in the lateral direction ( $x$-direction) is also approximately linear in relation with the subglottal pressure as illustrated in Figure 4.16.

The impact velocity is the most important factor when two bodies collide. However, it is very difficult to determine within the experimental setup of this work and there are no experimental data about it in the open literature.

\subsubsection{Glottal Collision Dynamics}

Figure 4.17 and Figure 4.18 show the contact area and impact force of the entire vocal fold during collision. The total contact area and contact force in the FEA model are in the range of available experimental results [50].

The impact phase occurs when one of the vocal folds first gets in contact with the opposing fold. Contact stresses between the vocal folds rise very quickly and reach peak values. During this phase, the vocal folds collision generates very large impact stresses (normal stress in this model). This pressure can be determined by,

$$
P=\frac{F}{A}
$$

$F=$ impact force

$A=$ impact area

An impact force is a large force applied over a short period of time. Such a force can have a large effect on the vocal folds. Using Newton's second law, the contact force can be calculated by

$$
f=m a=m \frac{\square v}{\square t}
$$

It is shown in Figure 4.12 that, the points near the midpoint have very high collision velocity, and shorter acceleration time period. Compared with the total impact phase, the mid area of vocal fold is the main injury risk area during the collision. Figure 4.22 
gives the contact stress of the selected points shown in Figure 4.21. An example of the collision velocity and period of node 250 is listed as follow,

$\square v=37.6 \mathrm{~mm} / \mathrm{s}, 47.5 \mathrm{~mm} / \mathrm{s}, 78.5 \mathrm{~mm} / \mathrm{s}(\mathrm{Ps}=800 \mathrm{~Pa}, 1000 \mathrm{~Pa}, 1500 \mathrm{~Pa})$

$\square t \approx 2.7^{-5} \mathrm{~s}$

Because the impact period is the same and the mass of each selected nodes are similar, the relationship between the subglottal pressure and the impact force is approximately linear. The relationships between the impact force and the superficial tissue mechanical stress can be obtained in the model collision process. Although, the total contact area varies with the subglottal pressure magnitudes, the contact area selected in Chapter 4 always contact with the opposing vocal fold. Furthermore, the contact area for each node in the selected area is constant during the vocal folds collision. This depends on ABAQUS mesh size rather than the subglottal pressure. Therefore, in the middle portion of the vocal folds, the relation between the peak impact stress and the subglottal pressure is approximately linear.

In medical theory, vocal nodule is believed to be caused by trauma. Furthermore, lots of observations illustrate that the vocal fold trauma occurs after collisions [56]. If the collision pressure is over $10 \mathrm{kPa}$, the impact stress may squish the vocal folds tissue and cause tissue failure.

As shown in Figure 4.22, when the subglottal pressure is over $800 \mathrm{~Pa}$, the peak impact stress will be over the vocal fold tissue damage level. Most of vocal nodule occurs at the middle of membranous fold [56]. The locations of the nodules match with the locations of the maximum impact force in this model. Therefore, the impact stress is assumed to be one of the most important factors of vocal nodule.

\subsection{Summary}

The FEA was validated by both EMA model results and published in-vivo experimental results. The eigenmodes show the vocal fold vibration directions at various excitation frequencies. In the dynamic analysis, the vocal folds closure process corresponds to clinical observation. In the mid area of the vocal folds inferior surface, the closure velocity, impact force and impact stress are linear with subglottal pressure. When the 
subglottal pressure is over $800 \mathrm{~Pa}$, the maximum impact stress will be more than $10 \mathrm{kPa}$, which is assumed to have the capacity to cause the vocal fold trauma. 


\section{Chapter 6 CONCLUSIONS AND FUTURE WORK}

\subsection{Conclusions}

The objective of this thesis was to develop a finite element model to simulate the process of vocal folds closure. The vocal fold vibrations and dynamic responses are investigated using an ABAQUS model which incorporates the inhomogeneity and anisotropy of the materials, the irregularity of the geometry and the layered structure. The initial aims set out in Chapter 1 have been achieved as following:

\section{1) Build a complete mathematical model to simulate the vocal folds and determine the geometry and material parameters in the model.}

The geometry of the model was based on Titze's theory [27, 29] and simplified for the FEA model. In the model, the vocal folds were divided into two layers: the cover part (assigned as an isotropic viscoelastic material), and the body part (considered as a transverse isotropic elastic material). Both of the material properties were obtained from experimental results in the literature. The experimental data was selected and converted to a Prony series form which can be used in ABAQUS. The boundary condition was determined by anatomical structure analysis. In this thesis, the vocal folds are assumed to be symmetric in a healthy situation.

\section{2) Validate the mathematical model for simulation.}

A silicone vocal fold model was developed as described in Chapter 3. Testing of the material mechanical properties is discussed in Chapter 3 and Chapter 4. The silicone material used has similar mechanical properties to the vocal fold tissue including density and elasticity. In the frequency analysis, the experimental results and finite element analysis results are in good agreement with each other. This validates the geometry and boundary conditions of the mathematical model. The theoretical results also agree with in-vivo experimental results in the literature. The agreement validates the material properties and layered structure in the mathematical model. Therefore, the 
developed mathematical model is a dependable model for frequency and dynamic analysis.

3) Compare the frequency results from the mathematical model and the experimental results of the silicone model and study the frequency characteristics of the vocal folds.

In the frequency analysis, the results were shown as eigenfrequencies and eigenmodes. The eigenfrequencies agreed with the resonance frequencies found in the experimental tests. The eigenmodes showed the resonance vibration directions at different excitation frequencies. This theory explains why some data was missed at the first resonance frequency during experiment testing. The results predict that the impact stress will increase dramatically when the air flow frequency is around $125 \mathrm{~Hz}$.

4) Compare the dynamic results from the mathematical model and the experimental results and study the dynamic response of the vocal folds..

In dynamic analysis, the glottal opening size and closing velocity were approximately linear with the subglottal pressure. The mid areas of the inferior surfaces of the vocal folds were found to suffer the maximum impact stress during glottal closure. These results agree with the experimental results in the literature. The impact stress analysis focused on these areas. The results showed that when the subglottal pressure was over $800 \mathrm{~Pa}$, the impact stress on that area may cause the vocal folds tissue failure.

\subsection{Future Work}

This model has the potential to simulate some vocal fold disorders. It is anticipated that the model will help to identify voice disorders such as vocal-fold paralysis and vocalfold nodules. However, in order to improve on this work, the following issues will need to be addressed:

1. In clinical observations, the vocal folds tissue failure develops after a long time due to both higher-pitched (higher frequency) voices and louder (higher amplitude) voice. In future, it is necessary to simulate periodical subglottal pressure to find the accumulated effect of impact stress during phonation. 
2. The shear force between the two vocal folds on the inferior surface is also a potential risk of tissue damage. This force should be considered if the friction ratio is determined in experimental test.

3. The vocal folds will be used to represent pathological and surgical alterations by changing some detail of the geometry and material properties. For example, the vocal fold nodule could be represented by adding a small mass onto the inferior surface of current the FEA model. 


\section{REFERENCES}

1. Learning about the voice mechanism. Voiceproblem.org. Available from: $<$ http://www.voiceproblem.org/anatomy/learning.asp\#> [Accessed 01/12/2005]

2. About the voice. Lions Voice Clinic. Available from:

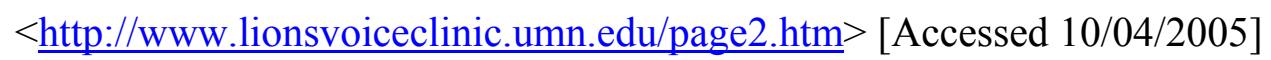

3. Aronson, A.E. Clinical voice disorders: Thieme; 1990.

4. Holmberg, E.B., Hillman, R.E., and Perkell, J.S. Glottal air-flow and transglottal air-pressure measurements for male and female speakers in soft, normal, and loud voice. Journal of Acoustical Society of America 1988;84 pp: $511-529$.

5. Titze, I.R. Mechanical stress in phonation. Journal of Voice 1994;8 (99-105) pp.

6. Greene, M.C.L. The voice and its disorders. Philadelphia: J. B. Lippincott; 1980.

7. Anatomy and physiology of the voice. The Milton J. Dance, Jr. Head \& Neck Rehabilitation Center. Available from: $<\underline{\text { http://www.gbmc.org/voice/anatomyphysiologyofthelarynx.cfm }>\text { [Accessed }}$ $05 / 12 / 2005]$

8. Gray, S.D., Alipour, F., Titze, I.R., and Hammond, T.H. Biomechanical and histologic observations of vocal fold fibrous proteins. The Annals of Otology, Rhinology \& Laryngology 2000;109 (1) pp: 77.

9. Hirano, M. Structure and vibratory behavior of the vocal folds. Tokyo: University of Tokyo Press; 1977.

10. Titze, I.R. Principles of voice production. Iowa City, Ia: National Center for Voice and Speech; 2000.

11. Hirano, M. Phonosurgery: Basic and clinical inverstigations. In: Official Report of the 76th Annual Convention of the Oto-Rhino-Laryngological Society of Japan, 1975. 
12. Titze, I.R. A framework for the study of vocal registers. Journal of Voice 1988;2 (3) pp: 183-194.

13. Kakita, Y., Hirano, M., and Ohmaru, K. Vocal fold physiology. Tokyo: University of Tokyo Press; 1981.

14. Flanagan, J., and Landgraf, L. Self-oscillating source for vocal-tract synthesizers. IEEE Trans. Audio Eletroacoust 1968;16 pp: 57-64.

15. Ishizaka, K., and Flanagan, J.L. Synthesis of voiced sounds from a two-mass model of the vocal cords. Bell Syst. Tech. J 1972;51 pp: 1233-1268.

16. Titze, I.R. The human vocal cords: A mathematical model. I. Phonetica 1973;28 pp: $129-170$.

17. Titze, I.R. The human vocal cords: A mathematical model. II. Phonetica 1974;29 pp: $1-21$.

18. Story, B.H., and Titze, I.R. Voice simulation with a body-cover model of the vocal folds. Journal of Acoustical Society of America 1995;97 (2) pp: 12491260.

19. Berry, D.A., and Titze, I.R. Interpretation of biomechanical simulations of normal and chaotic vocal fold oscillationswith empirical eigenfunctions. Journal of Acoustical Society of America 1994;95 (6) pp: 3595-3604.

20. Vries, M.P., Schutte, H.K., and Verkerke, G.J. Determination of parameters for lumped parameter models of the vocal folds using a finite-element method approach. Journal of Acoustical Society of America 1999;106 (6) pp: 36203628 .

21. Jiang, J.Y., Qiu, Q.J., and Xu, K.X. Modeling vocal-fold vibration via intergrating two-mass model with finite-element method. Journal of Biomedical Engineering 2005;22 (2) pp: 297-302.

22. Berry, D.A., and Titze, I.R. Normal modes in a continuum model of vocal fold tissues. Journal of Acoustical Society of America 1996;100 pp: 3345-3354. 
23. Courant, R. Variational method for the solution of problems of equilibrium and variations. Bulletin of the American Mathematical Society 1943;49 pp.

24. Brauer, J.R., ed. What every engineer should know about finite element analysis. New York: Marcel Dekker, Inc.; 1988.

25. Alipour, F., Berry, D.A., and Titze, I.R. A finite-element model of vocal-fold vibration. Acoustic Society of America 2000;108 (6) pp: 3003-3012.

26. Gunter, H.E. A mechanical model of vocal-fold collision with high spatial and temporal resolution. Acoustic Society of America 2003;113 (2) pp: 994-1000.

27. Scherer, R.C., Shinwari, D., Kenneth J. De Witt, Zhang, C., Kucinschi, B.R., and Afjeh, A.A. Intraglottal pressure profiles for a symmetric and oblique glottis with a divergence angle of 10 degrees. Journal of Acoustical Society of America 2001;109 (4) pp: 1616-1630.

28. Thomson, S.L., Mongeau, L., and Frankel, S.H. A physical model of the vocal folds. In: International Mechanical Engineering Congress; Washington, D.C.: ASME, 2003:1-2.

29. Mantha, S., Mongeau, L., and Siegmund, T. Dynamic digital image correlation of a dynmaic physical model of the vocal folds. In: International Mechanical Engineering Congress and Exposition; Orlando, Florida USA: ASME, 2005:1-2.

30. Titze, I.R., and Talkin, D.T. A theoretical study of the effects of various laryngeal configurations on the acoustics of phonation. Acoustic Society of America 1979;66 (1) pp: 60-74.

31. Min, Y.B., Titze, I.R., and Alipour, F. Stress-strain response of the human vocal ligament. Ann. Otol. Rhinol. Laryngol 1995;104 pp: 563-569.

32. Kaneko, T., Masuda, T., Akiki, S., Suzuki, H., Hayasaki, K., and komatsu,K. Resonance characteristics of the human vocal fold in vivo and in vitro by an impulse excitation. Laryngeal Function in Phonation and Respiration 1987 pp: 349-365.

33. Lakes, R.S. Viscoelastic solids. Boca Raton: CRC Press; 1999. 
34. Chan, R.W. Estimation of viscoelastic shear properties of vocal-fold tissues based on time-temperature superposition. Journal of Acoustical Society of America 2001;110 (3) pp: 1548-1561.

35. Cengel, Y.A. Heat transfer: A practical approach: McGraw-Hill College; 2002.

36. Chan, R.W., and Titze, I.R. Viscoelastic shear properties of human vocal fold mucosa: Mearsurement methodology and empirical results. Journal of Acoustical Society of America 1999;106 (4) pp: 2008-2021.

37. Chung, T.J. Applied continuum mechanics. New York, USA: Cambridge University Press; 1996.

38. Hunter, E.J., Titze, I.R., and Alipour, F. A three-dimensional model of vocal fold abduction/adduction. Journal of Acoustical Society of America 2003;115 (4) pp: 1747-1759.

39. Alipour, F., and Titze, I.R. Elastic models of vocal fold tissues. Journal of Acoustical Society of America 1991;90 (3) pp: 1326-31.

40. Importance of water in the diet. Available from: $<\underline{\text { http://www.chem.duke.edu/ jds/cruise chem/water/watdiet.html }>\text { Accessed }}$ $12 / 12 / 2005]$

41. Jiang, J., Lin, E., and Hanson, D.G. Voice disorders and phonosurgery. I. Vocal fold physiology. Otolaryngol. Clin. North Am 2000;33 pp: 699-718.

42. Chan, R.W., and Titze, I.R. Viscoelastic shear properties of human vocal fold mucosa: Theoretical characterization based on constitutive modeling. Journal of Acoustical Society of America 2000;107 (1) pp: 565-580.

43. He, J. M. and Fu, Z. F. Modal analysis. Oxford: Butterworth-Heinemann; 2001.

44. Harris, C. M., Piersol, A. G. Harris'shock and vibration handbook. New York: McGraw-Hill; 2002.

45. Biomechanics. Available from: $<$ http://en.wikipedia.org/wiki/Human_kinetics $>$ [Accessed 12/12/2005] 
46. Buddy, D.R., Allan, H., Frederick, S. and Jack, L. Biomaterials science: Elsevier, Inc.; 2004.

47. Condensation cured silicones. Available from:

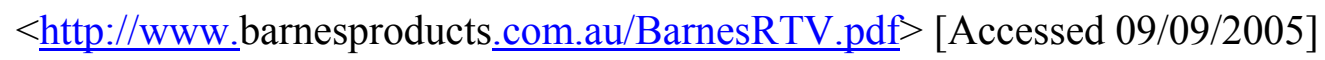

48. Hsiao, T.Y., Wang, C.L., Chen C.N., Hsieh F.J., and Shau, Y.W. Elasticity of human vocal folds measured in vivo using color doppler imaging. Ultrasound in Medicine and Biology 2002;28 (9) pp: 1145-1152.

49. Randestad, A., Lindholm, C.E., Fabian, P. Dimensions of the cricoid cartilage and the trachea. Laryngoscope 2000;110 (11) pp: 1957-1961.

50. Jack, J.J., and Titze, I.R. Measurement of vocal fold intraglottal pressure and impact stress. Journal of Voice 1994;8 (2) pp: 132-144.

51. Meirovitch, L. Fundamentals of vibrations. New York: McGraw-Hill; 2001.

52. Svec, J.G., Horacek, J., Sram, F., and Vesely, J. Resonance properties of the vocal folds: In vivo laryngoscopic investigation of the externally excited laryngeal vibrations. Journal of Acoustical Society of America 2000;108 (4) pp: 1397-1407.

53. Kaneko, T., Komatsu, K., Suzuki, H., Kanesaka, T., Masuda, T., Numata, T. and Naito, J. Mechanical properties of the human vocal fold - resonance characteristics in living humans and in excised larynges. In: Vocal fold physiology: Biomechanics, acoustics, and phonatory control (Titze, I.R. and Scherer, R.C. editors). Denver, CO: The Denver Center for the Performing Arts. 1983; pp. 304-317

54. Kaneko, T., Masuda, T., Shimada, A., Suzuki, H., Hayasaki, K. and Komatsu, K. Resonance characteristics of the human vocal folds in vivo and in vitro by an impulse excitation. In: Laryngeal function in phonation and respiration (Baer, T., Sasaki, C. and Harris, K. editors). Boston: Little, Brown. 1986; pp. 349-377

55. Kim, M.J., Hunter, E.J. and Titze, I.R. Comparison of human, canine, and ovine laryngeal dimensions. The Annals of Otology, Rhinology \& Laryngology 2004;113 (1) pp: 60. 
56. Nagata, K, Kurita, S, Yasumoto, S, Maeda, T, Kawasaki, H, Hirano, M. Vocal fold polyps and nodules. A 10-year review of 1,156 patients. Auris Nasus Larynx 1983;10 (27-35) pp. 


\section{APPENDIX}

APPENDIX I

APPENDIX II

APPENDIX III
Geometry data of the vocal folds model

Frequency domain data of viscoelastic mucosa

Silicone's elasticity test data 


\section{APPENDIX I}

(A) The lateral surface data points

Table AI.1 Lateral surface

\begin{tabular}{|c|c|c|}
\hline$x(\mathrm{~mm})$ & $y(\mathrm{~mm})$ & $\mathrm{z}(\mathrm{mm})$ \\
\hline 10 & 0 & $0-5$ \\
\hline 9.974 & 1 & $0-5$ \\
\hline 9.898 & 2 & $0-5$ \\
\hline 9.770 & 3 & $0-5$ \\
\hline 9.592 & 4 & $0-5$ \\
\hline 9.362 & 5 & $0-5$ \\
\hline 9.082 & 6 & $0-5$ \\
\hline 8.750 & 7 & $0-5$ \\
\hline 8.367 & 8 & $0-5$ \\
\hline 7.934 & 9 & $0-5$ \\
\hline 7.449 & 10 & $0-5$ \\
\hline 6.913 & 11 & $0-5$ \\
\hline 6.327 & 12 & $0-5$ \\
\hline 5.689 & 13 & $0-5$ \\
\hline 5.000 & 14 & $0-5$ \\
\hline
\end{tabular}

(B) The inferior surface's points are from Equation 2.3. It is a special plane at $\mathrm{z}=4.5 \mathrm{~mm}$, as all the results at that plane is 0 . So that, there is an additional plane $\mathrm{z}=4.5 \mathrm{~mm}$. The glottal width( $\mathrm{x}$ ) from Equation 2.3 on each plane $(\mathrm{z}=0 \mathrm{~mm}$ to $\mathrm{z}=5 \mathrm{~mm})$ is constant.

Table AI.2 The inferior surface's points from Equation 2.3

\begin{tabular}{|c|c|c|}
\hline$x(\mathrm{~mm})$ & $y(\mathrm{~mm})$ & $\mathrm{z}(\mathrm{mm})$ \\
\hline 4.5 & $0-14$ & 0 \\
\hline 2.8 & $0-14$ & 1 \\
\hline 1.5 & $0-14$ & 2 \\
\hline 0.6 & $0-14$ & 3 \\
\hline 0.1 & $0-14$ & 4 \\
\hline 0 & $0-14$ & 4.5 \\
\hline 0 & $0-14$ & 5 \\
\hline
\end{tabular}


(C) The inferior surface's points from Equation 2.2

Table AI.3 The inferior surface's points from Equation 2.2

\begin{tabular}{|c|c|c|c|c|c|c|c|c|}
\hline $\mathrm{x}(\mathrm{mm})$ & $\mathrm{y}(\mathrm{mm})$ & $\mathrm{z}(\mathrm{mm})$ & $\mathrm{x}(\mathrm{mm})$ & y (mm) & $\mathrm{z}(\mathrm{mm})$ & $\mathrm{x}(\mathrm{mm})$ & $\mathrm{y}(\mathrm{mm})$ & $\mathrm{z}(\mathrm{mm})$ \\
\hline 4.5 & 0 & 0 & 2.8 & 0 & 1 & 1.5 & 0 & 2 \\
\hline 4.489 & 1 & 0 & 2.793 & 1 & 1 & 1.496 & 1 & 2 \\
\hline 4.454 & 2 & 0 & 2.771 & 2 & 1 & 1.485 & 2 & 2 \\
\hline 4.397 & 3 & 0 & 2.736 & 3 & 1 & 1.466 & 3 & 2 \\
\hline 4.316 & 4 & 0 & 2.686 & 4 & 1 & 1.439 & 4 & 2 \\
\hline 4.213 & 5 & 0 & 2.621 & 5 & 1 & 1.404 & 5 & 2 \\
\hline 4.087 & 6 & 0 & 2.543 & 6 & 1 & 1.362 & 6 & 2 \\
\hline 3.937 & 7 & 0 & 2.45 & 7 & 1 & 1.313 & 7 & 2 \\
\hline 3.765 & 8 & 0 & 2.343 & 8 & 1 & 1.255 & 8 & 2 \\
\hline 3.57 & 9 & 0 & 2.221 & 9 & 1 & 1.19 & 9 & 2 \\
\hline 3.352 & 10 & 0 & 2.086 & 10 & 1 & 1.117 & 10 & 2 \\
\hline 3.111 & 11 & 0 & 1.936 & 11 & 1 & 1.037 & 11 & 2 \\
\hline 2.847 & 12 & 0 & 1.771 & 12 & 1 & 0.949 & 12 & 2 \\
\hline 2.56 & 13 & 0 & 1.593 & 13 & 1 & 0.853 & 13 & 2 \\
\hline 2.25 & 14 & 0 & 1.4 & 14 & 1 & 0.75 & 14 & 2 \\
\hline $\mathrm{x}(\mathrm{mm})$ & $\mathrm{y}(\mathrm{mm})$ & 1) $\mathrm{z}(\mathrm{mm})$ & $\mathrm{x}(\mathrm{mm})$ & $\mathrm{y}(\mathrm{mm})$ & $\mathrm{z}(\mathrm{mm})$ & \multirow{16}{*}{\multicolumn{3}{|c|}{$\mathrm{x}=0 \mathrm{~mm}$ at $\mathrm{z}=5 \mathrm{~mm}$}} \\
\hline 0.6 & 0 & 3 & 0.1 & 0 & 4 & & & \\
\hline 0.598 & 1 & 3 & 0.1 & 1 & 4 & & & \\
\hline 0.594 & 2 & 3 & 0.099 & 2 & 4 & & & \\
\hline 0.586 & 3 & 3 & 0.098 & 3 & 4 & & & \\
\hline 0.576 & 4 & 3 & 0.096 & 4 & 4 & & & \\
\hline 0.562 & 5 & 3 & 0.094 & 5 & 4 & & & \\
\hline 0.545 & 6 & 3 & 0.091 & 6 & 4 & & & \\
\hline 0.525 & 7 & 3 & 0.087 & 7 & 4 & & & \\
\hline 0.502 & 8 & 3 & 0.084 & 8 & 4 & & & \\
\hline 0.476 & 9 & 3 & 0.079 & 9 & 4 & & & \\
\hline 0.447 & 10 & 3 & 0.074 & 10 & 4 & & & \\
\hline 0.415 & 11 & 3 & 0.069 & 11 & 4 & & & \\
\hline 0.38 & 12 & 3 & 0.063 & 12 & 4 & & & \\
\hline 0.341 & 13 & 3 & 0.057 & 13 & 4 & & & \\
\hline 0.3 & 14 & 3 & 0.05 & 14 & 4 & & & \\
\hline
\end{tabular}




\section{APPENDIX II}

Frequency domain Storage and Loss modulus of vocal fold cover (59-year-old-male)

Table AII.1

\begin{tabular}{|c|c|c|c|c|c|c|}
\hline $\mathrm{f}$ & loss & wR & ws' & storage & $\mathrm{wS}$ & wr' \\
\hline 0.01 & 1.9 & & 0.99641509 & 7.8 & 0.985283 & 0.014717 \\
\hline 0.02 & 1.9 & 0.00358491 & 0.99641509 & 8 & 0.984906 & 0.015094 \\
\hline 0.03 & 1.95 & 0.00367925 & 0.99632075 & 8.05 & 0.984811 & 0.015189 \\
\hline 0.04 & 1.96 & 0.00369811 & 0.99630189 & 8.1 & 0.984717 & 0.015283 \\
\hline 0.05 & 2 & 0.00377358 & 0.99622642 & 8.3 & 0.98434 & 0.01566 \\
\hline 0.07 & 2.03 & 0.00383019 & 0.99616981 & 8.6 & 0.983774 & 0.016226 \\
\hline 0.1 & 2.2 & 0.00415094 & 0.99584906 & 9 & 0.983019 & 0.016981 \\
\hline 0.2 & 2.5 & 0.00471698 & 0.99528302 & 9.6 & 0.981887 & 0.018113 \\
\hline 0.3 & 2.8 & 0.00528302 & 0.99471698 & 10 & 0.981132 & 0.018868 \\
\hline 0.4 & 3 & 0.02641509 & 0.99433962 & 10.05 & 0.981038 & 0.018962 \\
\hline 0.5 & 3.2 & 0.00603774 & 0.99396226 & 10.6 & 0.98 & 0.02 \\
\hline 0.6 & 3.35 & 0.00632075 & 0.99367925 & 10.8 & 0.979623 & 0.020377 \\
\hline 0.7 & 3.5 & 0.00660377 & 0.99339623 & 11 & 0.979245 & 0.020755 \\
\hline 1 & 3.7 & 0.00698113 & 0.99301887 & 11.5 & 0.978302 & 0.021698 \\
\hline 2 & 4.9 & 0.00924528 & 0.99075472 & 14 & 0.973585 & 0.026415 \\
\hline 3 & 5.9 & 0.01113208 & 0.98886792 & 15 & 0.971698 & 0.028302 \\
\hline 4 & 6.1 & 0.01150943 & 0.98849057 & 18 & 0.966038 & 0.033962 \\
\hline 5 & 6.1 & 0.01150943 & 0.98849057 & 20.5 & 0.961321 & 0.038679 \\
\hline 6 & 6.1 & 0.01150943 & 0.98849057 & 23 & 0.956604 & 0.043396 \\
\hline 7 & 6.2 & 0.01169811 & 0.98830189 & 24 & 0.954717 & 0.045283 \\
\hline 8 & 6.3 & 0.01188679 & 0.98811321 & 25 & 0.95283 & 0.04717 \\
\hline 9 & 6.8 & 0.01283019 & 0.98716981 & 26 & 0.950943 & 0.049057 \\
\hline
\end{tabular}




\section{APPENDIX III}

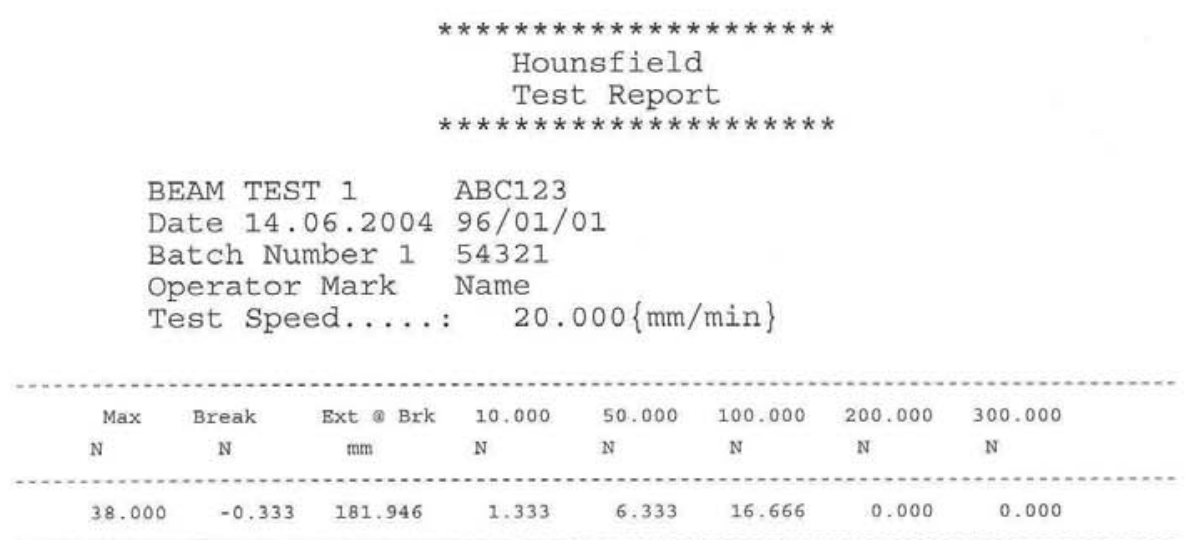

Beam size: $45 \mathrm{~mm}^{*} 11 \mathrm{~mm}{ }^{*} 7 \mathrm{~mm}$ (length*width*depth)

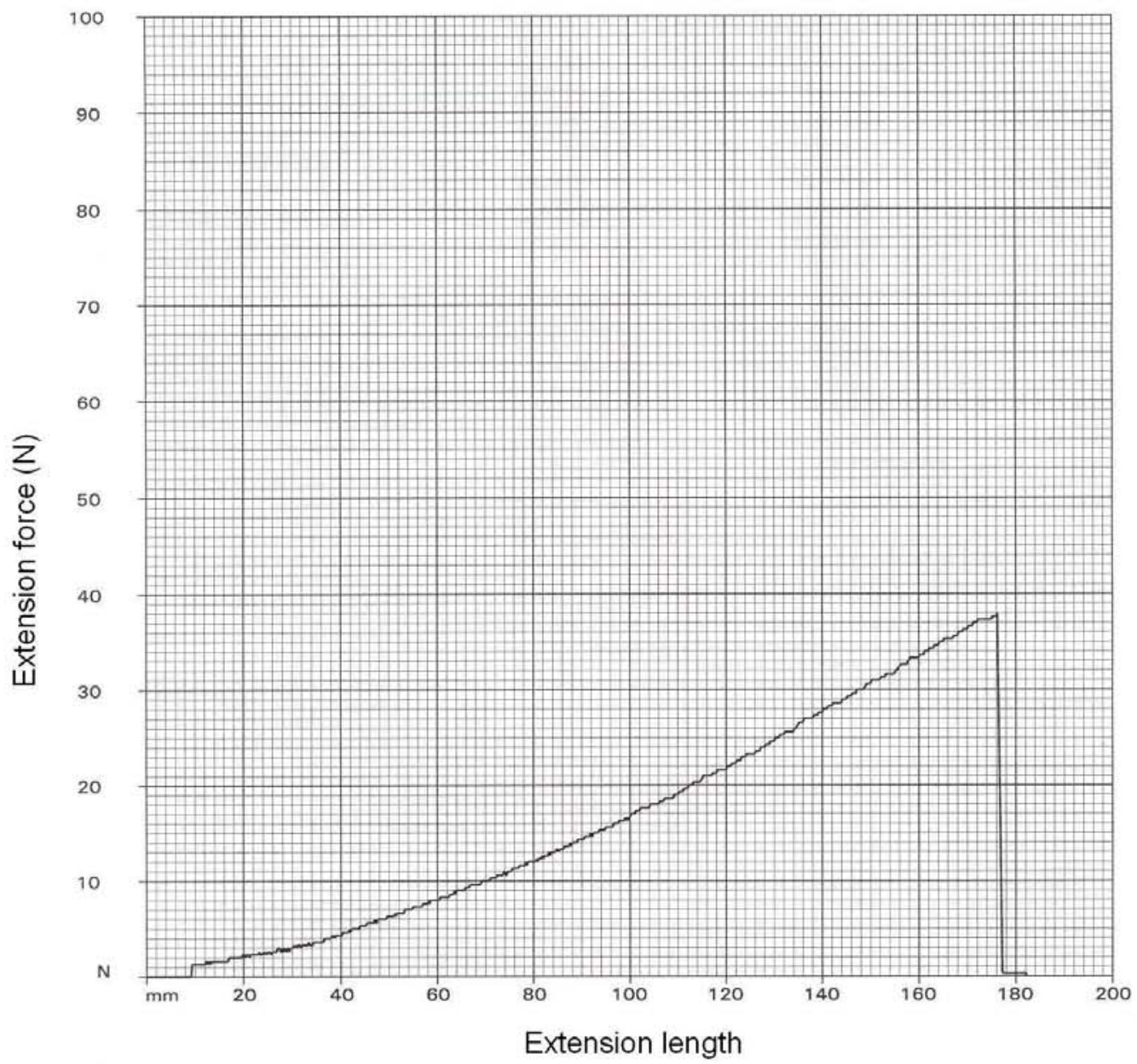

Figure AIII-1 Extension-force test one 
Hounsfield

Test Report

BEAM TEST 1 ABC123

Date 14.06.2004 96/01/01

Batch Number 154321

Operator Mark Name

Test Speed....: $50.000\{\mathrm{~mm} / \mathrm{min}\}$

$\begin{array}{lllllll}\text { Max Break } & \text { Ext } \otimes \text { Brk } & 10.000 & 50.000 & 100.000 & 200.000 & 300.000\end{array}$

$\begin{array}{llllllll}\mathrm{N} & \mathrm{N} & \mathrm{mm} & \mathrm{N} & \mathrm{N} & \mathrm{N} & \mathrm{N} & \mathrm{N}\end{array}$

$\begin{array}{llllllll}27.666 & -1.000 & 166.015 & 1.000 & 6.666 & 16.333 & 0.000 & 0.000\end{array}$

Beam size: $50 \mathrm{~mm} * 10 \mathrm{~mm} * 7 \mathrm{~mm}$ (length*width*depth)

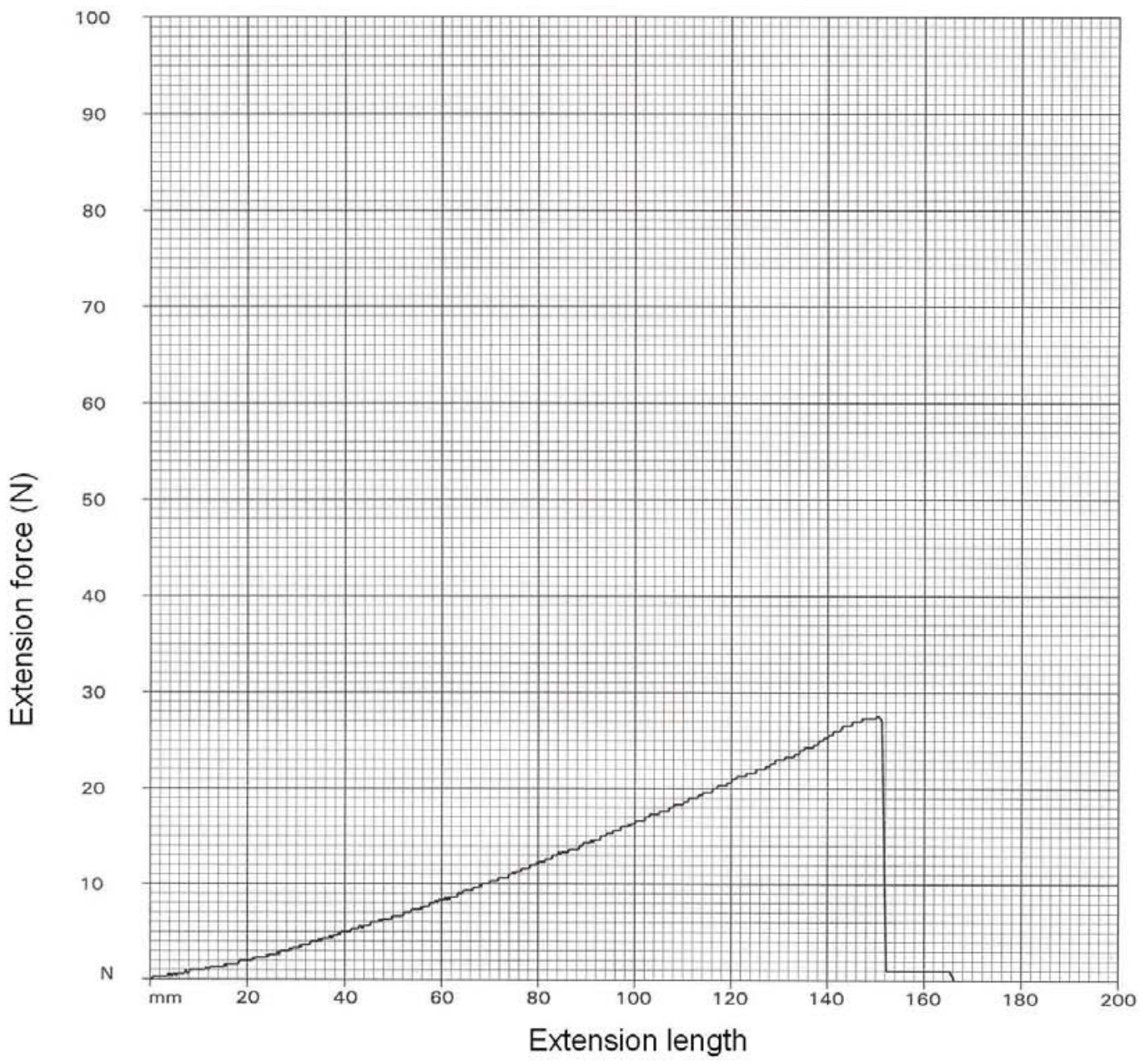

Figure AIII-2 Extension-force test two 
Hounsfield

Test Report

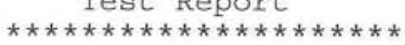

BEAM TEST 1 ABC123

Date 14.06.2004 96/01/01

Batch Number 154321

Operator Mark Name

Test Speed....: $\quad 50.000\{\mathrm{~mm} / \mathrm{min}\}$

\begin{tabular}{|c|c|c|c|c|c|c|c|}
\hline $\operatorname{Max}$ & Break & Ext $\cong \mathrm{Brk}$ & 10.000 & 50.000 & 100.000 & 200.000 & 300.000 \\
\hline $\mathrm{N}$ & N & $\mathrm{mm}$ & N & N & $\mathrm{N}$ & N & $\mathrm{N}$ \\
\hline 12.666 & -1.000 & 109.282 & 1.000 & 6.333 & -1.000 & 0.000 & 0.000 \\
\hline
\end{tabular}

Beam size: $60 \mathrm{~mm} * 13.5 \mathrm{~mm}^{*} 8.5 \mathrm{~mm}$ (length*width*depth)

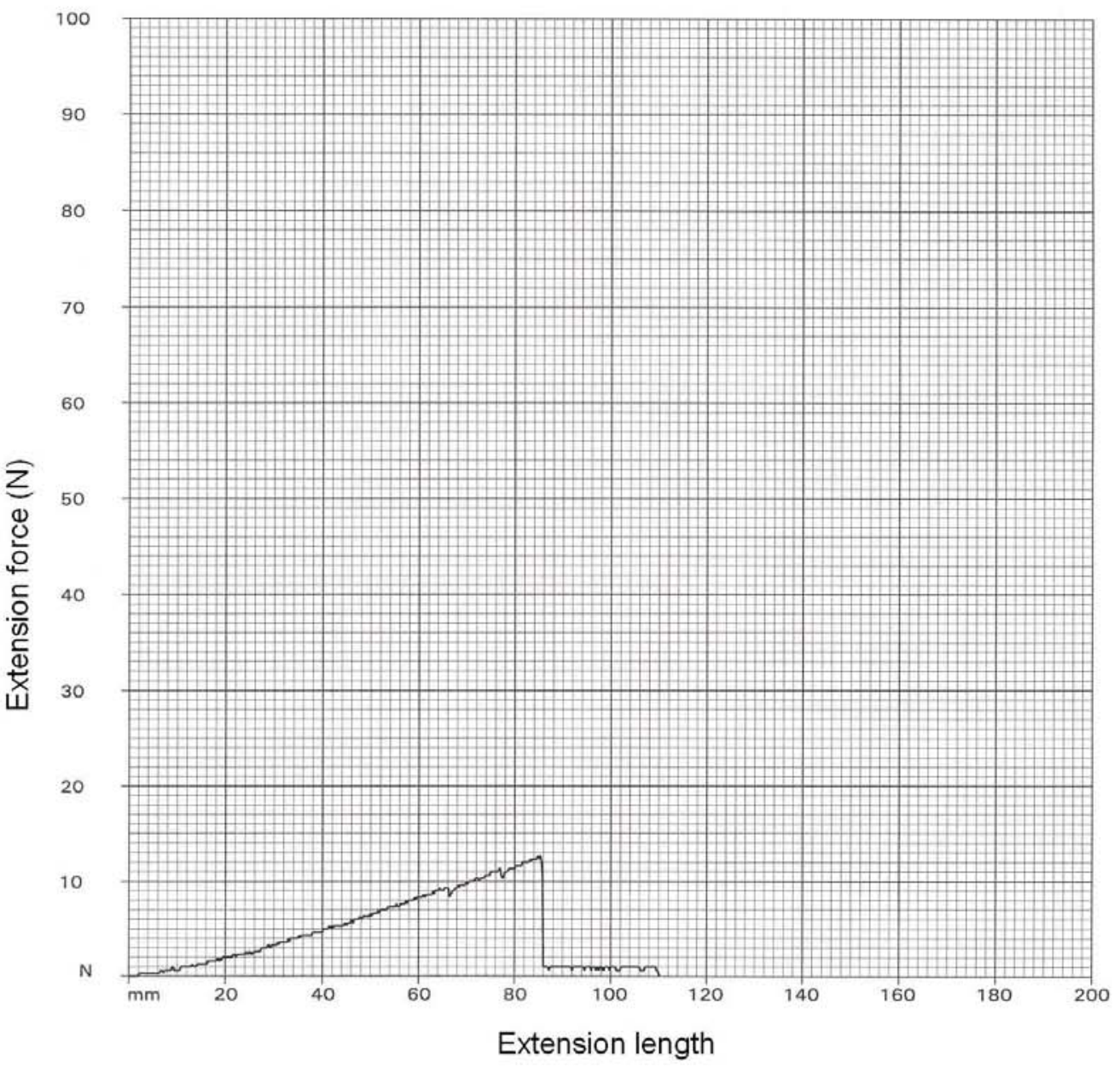

Figure AIII-3 Extension-force test three 
Hounsfield

Test Report
$* * * * * * * * * * * * * * * * * * * * *$

BEAM TEST 1 ABC123

Date 14.06.2004 96/01/01

Batch Number 154321

Operator Mark Name

Test Speed....: $50.000\{\mathrm{~mm} / \mathrm{min}\}$

\begin{tabular}{|c|c|c|c|c|c|c|c|}
\hline $\operatorname{Max}$ & Break & Ext Brk & 10.000 & 50.000 & 100.000 & 200.000 & 300.000 \\
\hline N & $\mathrm{N}$ & $\mathrm{mm}$ & $\mathrm{N}$ & N & $\mathrm{N}$ & $\mathrm{N}$ & N \\
\hline 25.333 & -1.000 & 144.747 & 1.000 & 4.666 & 15.666 & 0.000 & 0.000 \\
\hline
\end{tabular}

Beam size: $60 \mathrm{~mm}{ }^{*} 13.5 \mathrm{~mm}^{*} 8 \mathrm{~mm}$ (length ${ }^{*}$ width*depth)

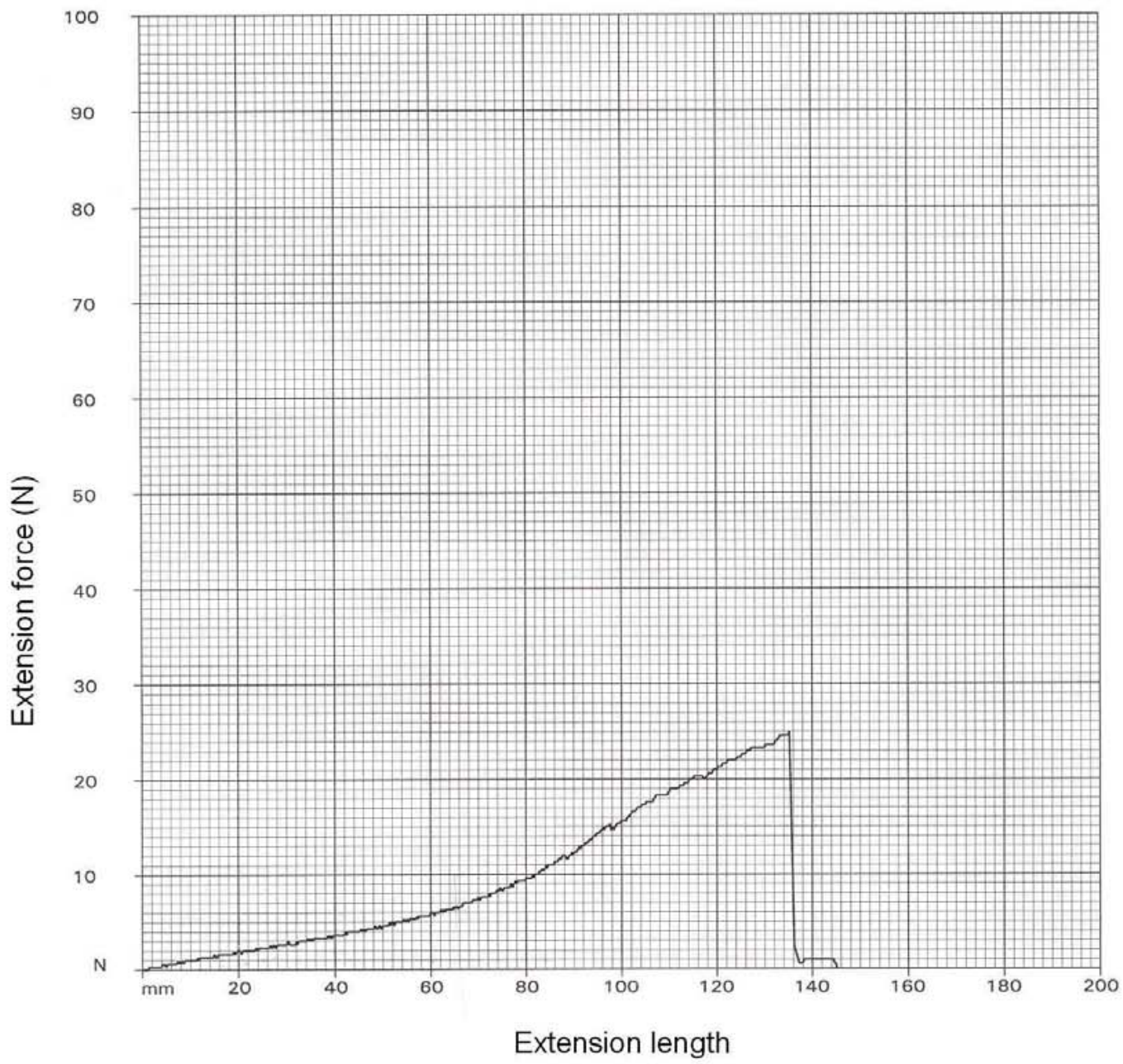

Figure AIII-4 Extension-force test four 

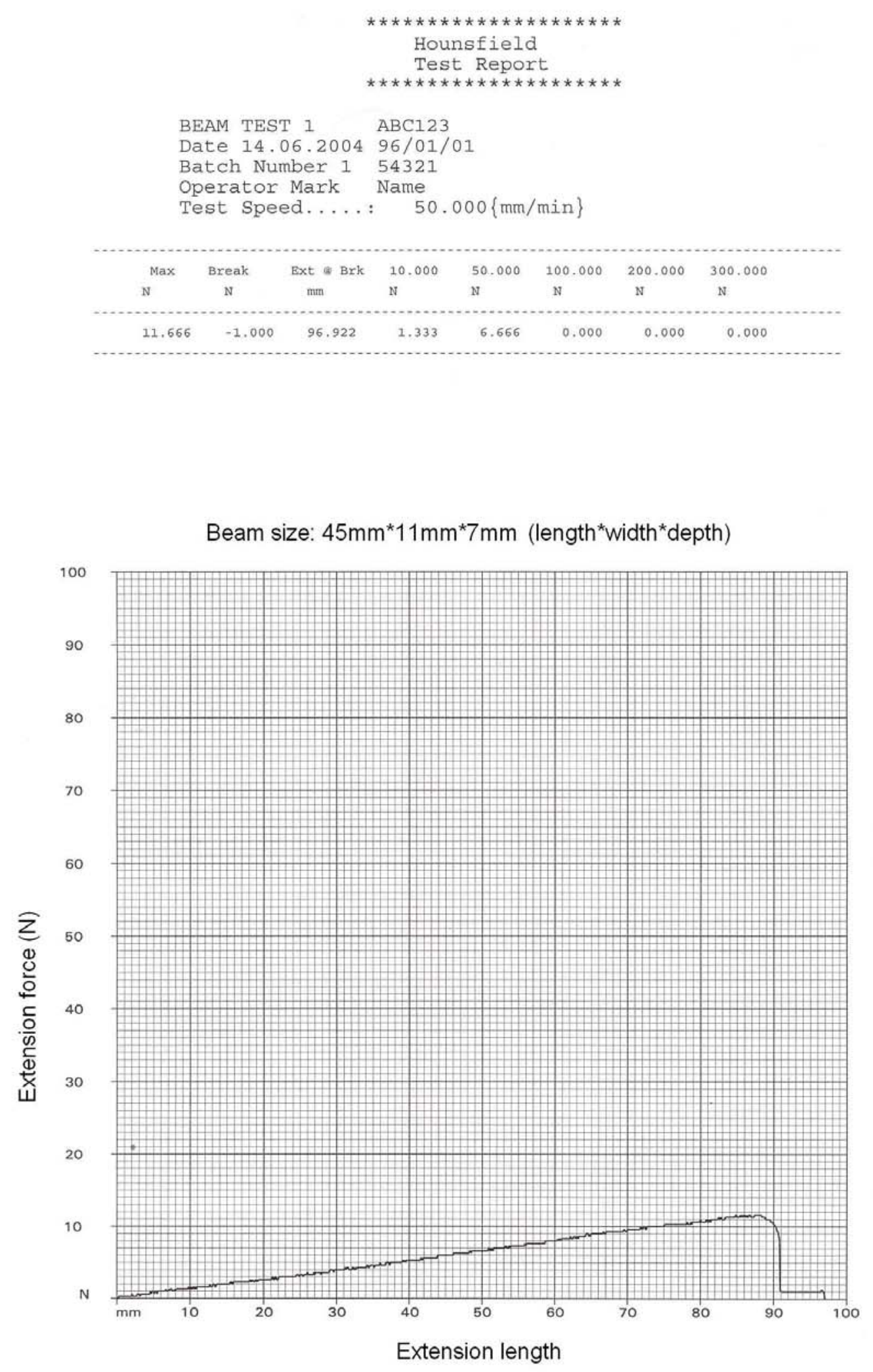

Figure AIII-5 Extension-force test five 
(A) The stress-strain relationship test data of test 1

Table AIII-1

\begin{tabular}{|c|c|c|}
\hline Force $(\mathrm{N})$ & Stress $1(\mathrm{~Pa})$ & Strain 1 \\
\hline 0 & 0 & 0 \\
\hline 1 & 12.98701 & 0.222222 \\
\hline 2 & 25.97403 & 0.444444 \\
\hline 3 & 38.96104 & 0.666667 \\
\hline 4.3 & 55.84416 & 0.888889 \\
\hline 6.2 & 80.51948 & 1.111111 \\
\hline 8 & 103.8961 & 1.333333 \\
\hline 10 & 129.8701 & 1.555556 \\
\hline 12 & 155.8442 & 1.777778 \\
\hline 14.2 & 184.4156 & 2 \\
\hline 16.5 & 214.2857 & 2.222222 \\
\hline 19 & 246.7532 & 2.444444 \\
\hline 22 & 285.7143 & 2.666667 \\
\hline 25 & 324.6753 & 2.888889 \\
\hline 28 & 363.6364 & 3.111111 \\
\hline 31 & 402.5974 & 3.333333 \\
\hline 33.1 & 429.8701 & 3.555556 \\
\hline
\end{tabular}

(B) The stress-strain relationship test data of test 2

Table AIII-2

\begin{tabular}{|c|c|c|}
\hline Force $(\mathrm{N})$ & Stress2 $(\mathrm{Pa})$ & Strain2 \\
\hline 0 & 0 & 0 \\
\hline 1 & 13.60544 & 0.222222 \\
\hline 2 & 27.21088 & 0.444444 \\
\hline 3.2 & 43.53741 & 0.666667 \\
\hline 5 & 68.02721 & 0.888889 \\
\hline 6.7 & 91.15646 & 1.111111 \\
\hline 8.2 & 111.5646 & 1.333333 \\
\hline 10.2 & 138.7755 & 1.555556 \\
\hline 12.2 & 165.9864 & 1.777778 \\
\hline 14.2 & 193.1973 & 2 \\
\hline 16.5 & 224.4898 & 2.222222 \\
\hline 18.3 & 248.9796 & 2.444444 \\
\hline 21.7 & 295.2381 & 2.666667 \\
\hline 23 & 312.9252 & 2.888889 \\
\hline 25.5 & 346.9388 & 3.111111 \\
\hline 28 & 380.9524 & 3.333333 \\
\hline
\end{tabular}


(C) The stress-strain relationship test data of test 3

Table AIII-3

\begin{tabular}{|c|c|c|}
\hline Force $(\mathrm{N})$ & Stress3 $(\mathrm{Pa})$ & Strain3 \\
\hline 0 & 0 & 0 \\
\hline 1 & 13.60544 & 0.2 \\
\hline 2 & 27.21088 & 0.4 \\
\hline 3.2 & 43.53741 & 0.6 \\
\hline 5 & 68.02721 & 0.8 \\
\hline 6.3 & 85.71429 & 1 \\
\hline 8.2 & 111.5646 & 1.2 \\
\hline 10 & 136.0544 & 1.4 \\
\hline 11.5 & 156.4626 & 1.6 \\
\hline
\end{tabular}

(D) The stress-strain relationship test data of test 4

Table AIII-4

\begin{tabular}{|c|c|c|}
\hline Force $(\mathrm{N})$ & Stress4 $(\mathrm{Pa})$ & Strain4 \\
\hline 0 & 0 & 0 \\
\hline 1 & 8.714597 & 0.166667 \\
\hline 2 & 17.42919 & 0.333333 \\
\hline 2.8 & 24.40087 & 0.5 \\
\hline 3.7 & 32.24401 & 0.666667 \\
\hline 4.5 & 39.21569 & 0.833333 \\
\hline 6 & 52.28758 & 1 \\
\hline 7.4 & 64.48802 & 1.166667 \\
\hline 9.5 & 82.78867 & 1.333333 \\
\hline 12 & 104.5752 & 1.5 \\
\hline 15.5 & 135.0763 & 1.666667 \\
\hline 19 & 165.5773 & 1.833333 \\
\hline 21 & 183.0065 & 2 \\
\hline 23.5 & 204.793 & 2.166667 \\
\hline
\end{tabular}

(E) The stress-strain relationship test data of test 5

Table AIII-5

\begin{tabular}{|c|c|c|}
\hline Force $(\mathrm{N})$ & Stress5 $(\mathrm{Pa})$ & Strain5 \\
\hline 0 & 0 & 0 \\
\hline 1.2 & 11.11111 & 0.166667 \\
\hline 2.6 & 24.07407 & 0.333333 \\
\hline 4 & 37.03704 & 0.5 \\
\hline 5.2 & 48.14815 & 0.666667 \\
\hline 6.8 & 62.96296 & 0.833333 \\
\hline 8 & 74.07407 & 1 \\
\hline 9.5 & 87.96296 & 1.166667 \\
\hline 10.5 & 97.22222 & 1.333333 \\
\hline 12 & 111.1111 & 1.5 \\
\hline
\end{tabular}


